Aus der Klinik für Anästhesiologie

(Prof. Dr. med. K. Meissner)

der Medizinischen Fakultät der Universität Göttingen

\title{
Qualitätsverbesserung in der
}

\section{Schmerztherapie konservativer (nicht operativer) Patienten (QUIKS)}

Ein Modul des QUIPS-Projekts zum Benchmarking der Schmerztherapie bei konservativen Patienten

\author{
INAUGURAL-DISSERTATION \\ zur Erlangung des Doktorgrades \\ der Medizinischen Fakultät der \\ Georg-August-Universität zu Göttingen
}

vorgelegt von

Christopher Bertemes

aus

Essen

Göttingen 2021 
Dekan:

Betreuungsausschuss

Betreuer:

Ko-Betreuer:

Prüfungskommission

Referent:

Ko-Referent:
Prof. Dr. med. W. Brück

Prof. Dr. med. F. Petzke

Prof. Dr. med. S. Kunsch

Prof. Dr. med. F. Petzke

Prof. Dr. med. S. Kunsch

Datum der mündlichen Prüfung: 18.02.2021 
Hiermit erkläre ich, die Dissertation mit dem Titel "Qualitätsverbesserung in der Schmerztherapie konservativer (nicht operativer) Patienten (QUIKS): ein Modul des QUIPS-Projekts zum Benchmarking der Schmerztherapie bei konservativen Patienten" eigenständig angefertigt und keine anderen als die von mir angegebenen Quellen und Hilfsmittel verwendet zu haben.

Göttingen, den 18.02.2021

(Unterschrift) 
Die Daten, auf denen die vorliegende Arbeit basiert, wurden teilweise publiziert:

Erlenwein J, Bertemes C, Kunsch S, Göttermann A, Komann M, Sturm L, Petzke F, Meißner W (2020): Qualitätsverbesserung im konservativen Schmerzmanagement (QUIKS). Schmerz 34, 52-64 


\section{Inhaltsverzeichnis}

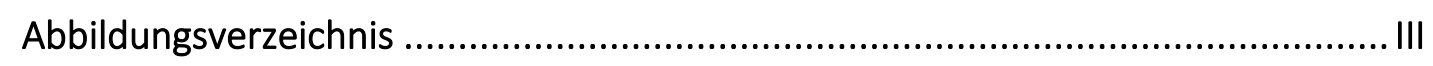

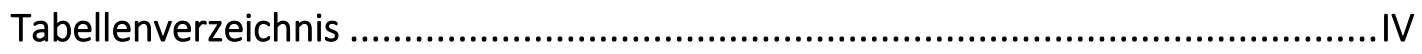

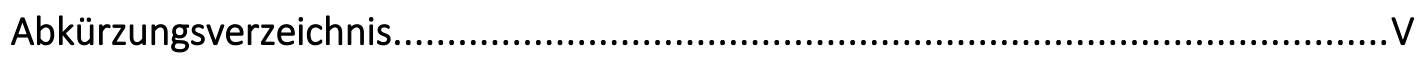

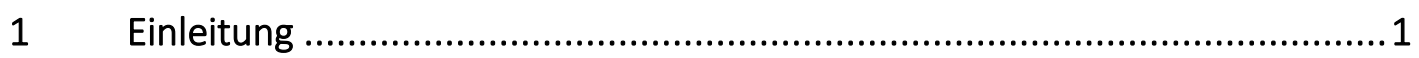

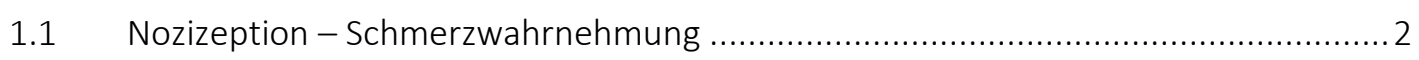

1.2 Auswirkungen von Schmerzen auf den Heilungsverlauf ........................................... 4

1.3 Stand der Forschung zur Qualität der Schmerztherapie ..................................... 6

1.3.1 Qualitätsmanagement und Benchmarking ....................................................

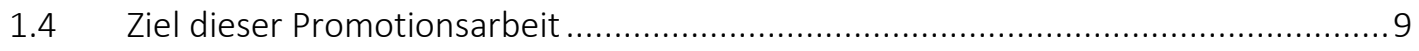

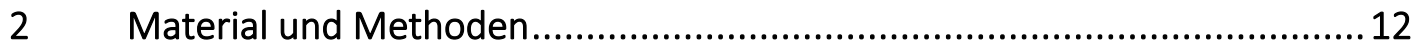

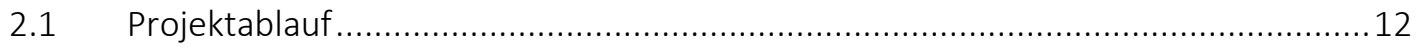

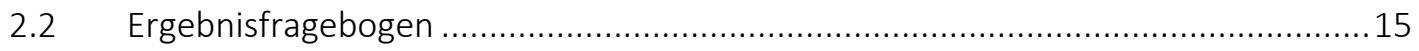

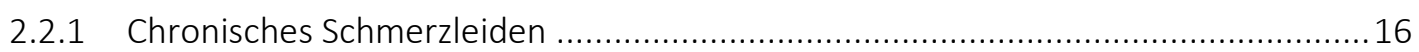

2.2.2 Schmerzentitäten und Schmerzintensitäten ................................................... 16

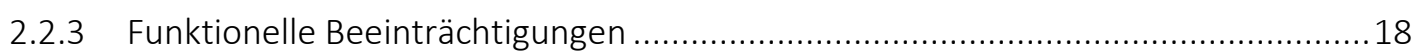

2.2.4 Therapiebedingte Nebenwirkungen oder weitere Symptome ...............................19

2.2.5 Patienteninformation, Patientenbewertung und Zufriedenheit ..............................20

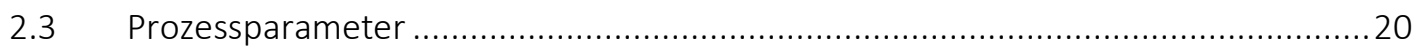

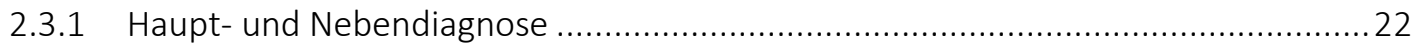

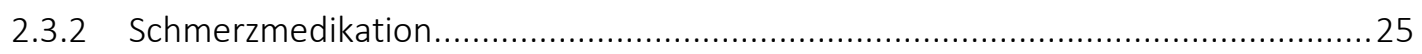

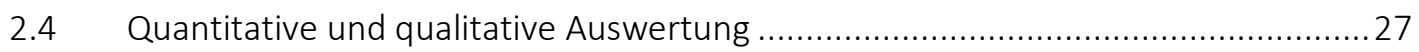

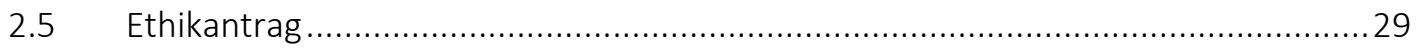

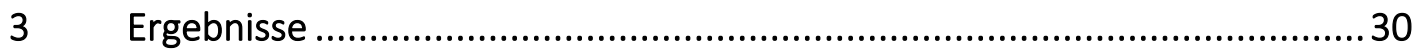

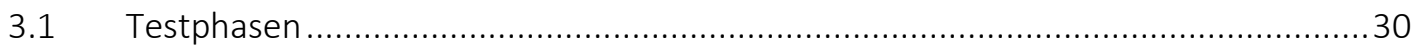

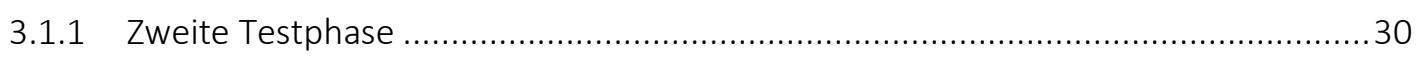

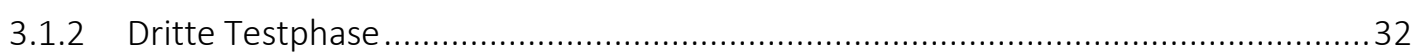

3.2 Projektphase: Quantitative Auswertung des Ergebnisfragebogens .........................32

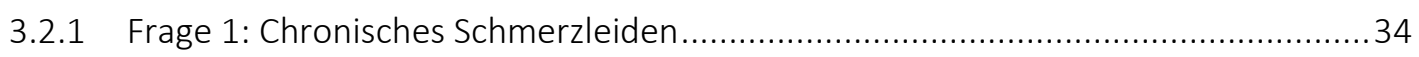

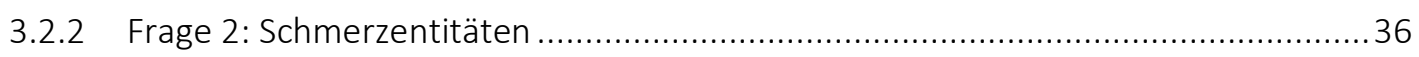

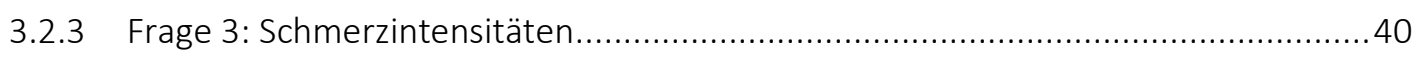

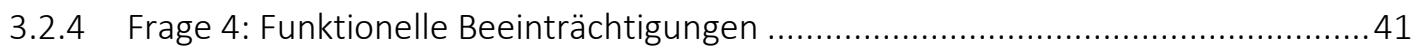

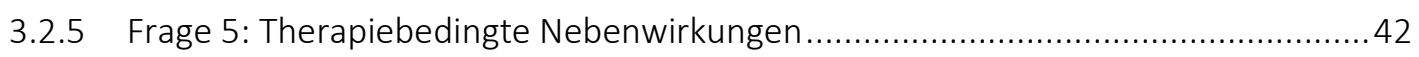

3.2.6 Frage 6, 7 und 8: Patienteninformation und Zufriedenheit .................................42

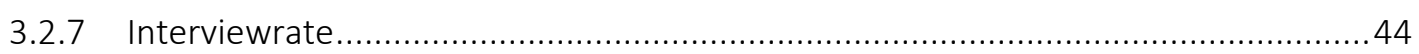

3.2.8 Subjektive Einschätzungen der Befragenden ................................................... 45 
3.3 Projektphase: Quantitative Auswertung der Prozessparameter ............................45

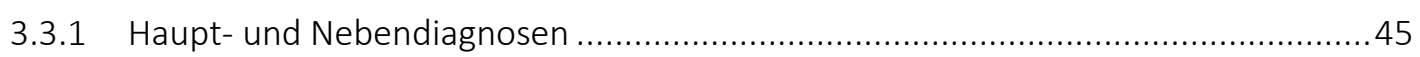

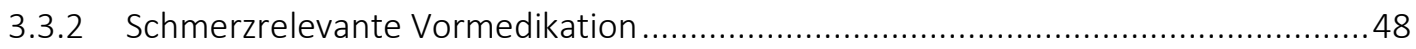

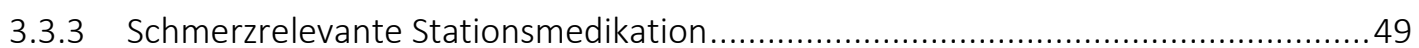

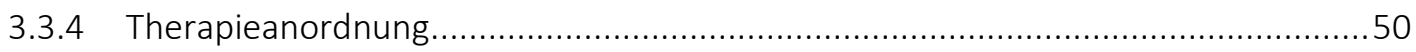

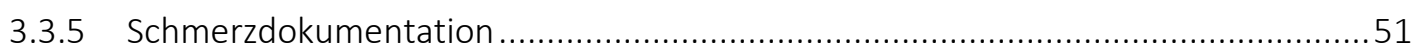

3.4 Qualitative Auswertung des Erhebungsinstruments ................................................52

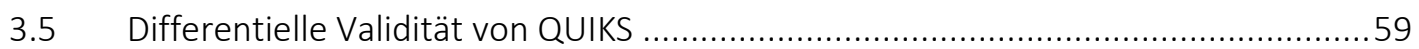

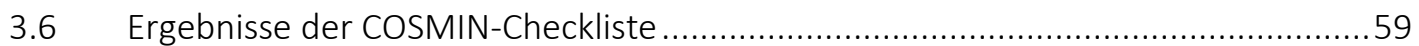

3.7 Chronische Schmerzen als Beispiel einer inhaltlichen Auswertung ........................61

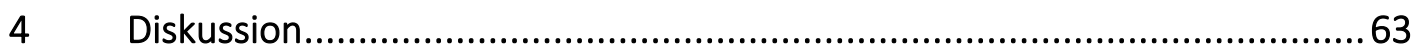

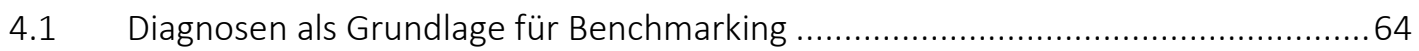

4.2 Spezielle Analyse im Literaturvergleich ...................................................................66

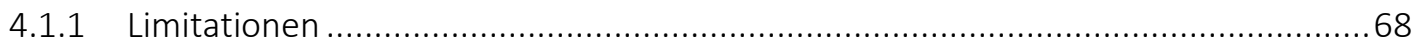

4.1.2 Bewertung mit Hilfe der COSMIN-Checkliste ........................................................69

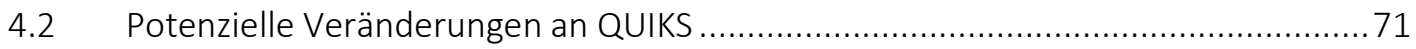

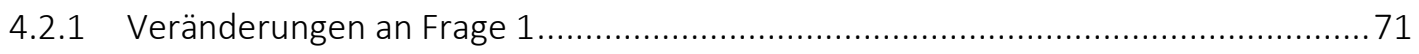

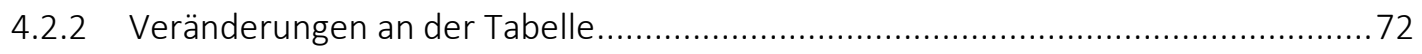

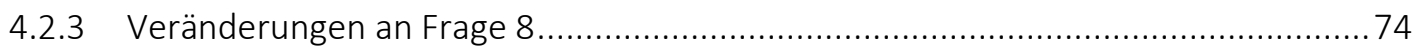

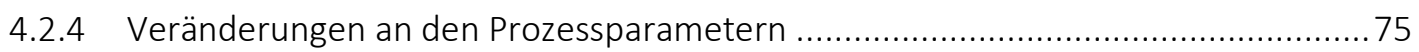

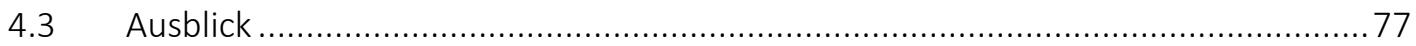

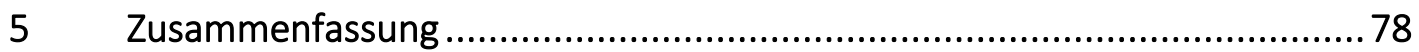

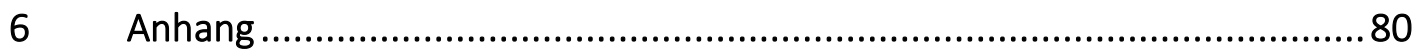

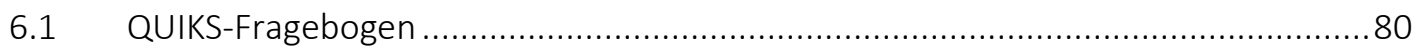

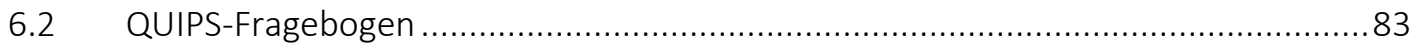

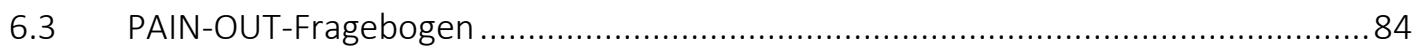

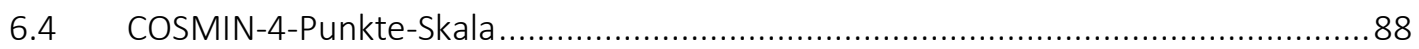

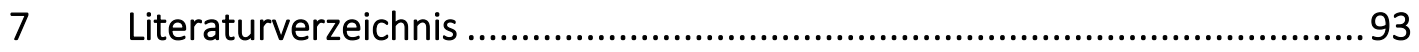




\section{Abbildungsverzeichnis}

Abbildung 1: Frage zu vorbestehenden chronischen Schmerzen mit drei abhängigen Teilfragen

Abbildung 2: Frage $2 a$ und die Liste der möglichen Schmerzentitäten .................................17

Abbildung 3: Angaben zu empfundenen Schmerzintensitäten......................................... 18

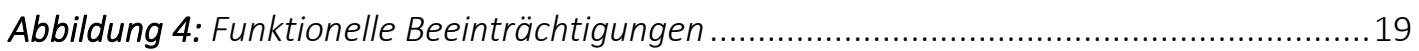

Abbildung 5: Therapiebedingte Nebenwirkungen oder weitere Symptome .........................19

Abbildung 6: Fragen zur Patienteninformation und Patientenzufriedenheit .......................20

Abbildung 7: Erhobene Prozessparameter........................................................21

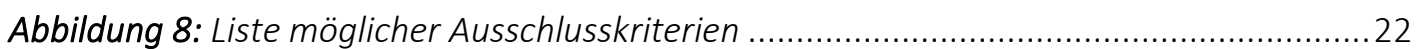

Abbildung 9: Übersicht der Wortdiagnosen innerhalb der spezifischen Organsystem.........24

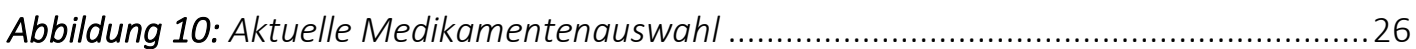

Abbildung 11: Individuelle Therapieanordnung (Basis- und/oder Bedarfsmedikation) und Schmerzdokumentation .................................................................... 27

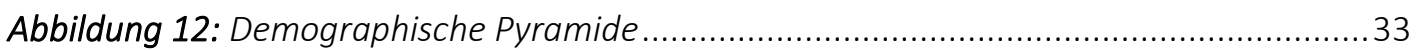

Abbildung 13: Geschlechtsspezifische Angaben zu empfundenen Schmerzintensitäten bei der Klinikaufnahme und bei Prozeduren während des Klinikaufenthalts .................39

Abbildung 14: Geschlechterspezifische Darstellung für "Ruhe-, Belastungs- und stärkster Schmerz" in den vergangenen 24 Stunden vor der Befragung

Abbildung 15: Angaben zur Patientenzufriedenheit mittels NRS, jeweils bei Patienten mit chronischen Schmerzen, mit Schmerzen bei der Aufnahme und mit einer Schmerzangabe in der Indikatorfrage $3 c$.

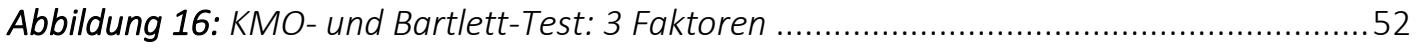

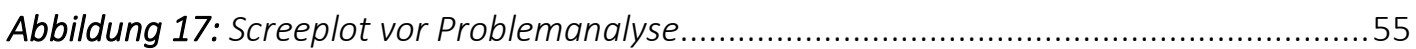

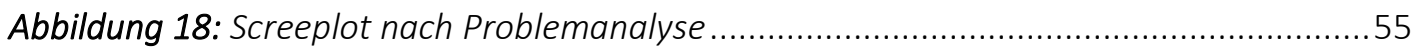

Abbildung 19: Grafische Darstellung der Schmerzintensitäten der Ruhe- und Belastungsschmerzen zwischen Patienten mit und ohne chronische Schmerzen ....................62

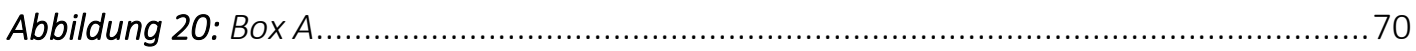

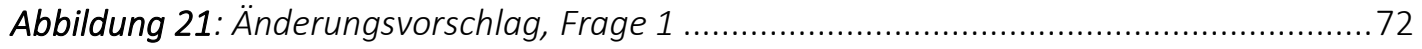

Abbildung 22: Überarbeitete Liste der möglichen Schmerzentitäten .................................74

Abbildung 23: Überarbeitete Frage zur Patientenzufriedenheit ......................................75 


\section{Tabellenverzeichnis}

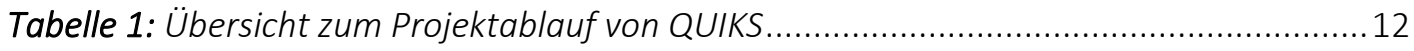

Tabelle 2: Übersicht über die Teilnahmekriterien ................................................................14

Tabelle 3: Patientenverteilung innerhalb der verschiedenen Fachdisziplinen der Inneren Me-

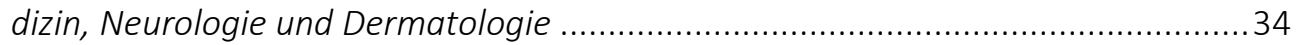

Tabelle 4: Gesamtauswertung der Frage 1: Vorbestehendes chronisches Schmerzleiden ....35

Tabelle 5: Eine Übersicht mit den Angaben zu den empfundenen Schmerzentitäten in der Klinik unabhängig von der Aufnahmediagnose.

Tabelle 6: Zugehörigkeiten der jeweiligen Hauptdiagnosen zu den spezifischen Organsystemen.

Tabelle 7: Übersicht der spezifischen Hauptdiagnosen >1\% Häufigkeit ...............................48

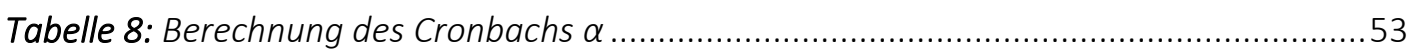

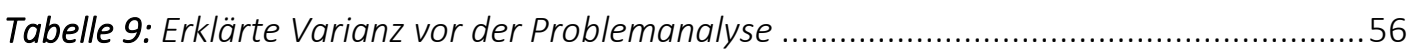

Tabelle 10: Erklärte Varianz nach der Problemanalyse .........................................................56

Tabelle 11: Deskriptive Statistik und Faktorenanalyse mit drei festgelegten Faktoren .........57

Tabelle 12: Deskriptive Statistik und Faktorenanalyse nach Entfernung der Fragen 6 bis 8.58

Tabelle 13: Ergebnis des Mann-Whitney-U-Tests .60

Tabelle 14: Übersicht über die Beantwortung von möglichen Schmerzindikatorfragen (2a, 2b, 3c und 8) durch Patienten mit chronischen und nicht chronischen Schmerzen ..62 


\section{Abkürzungsverzeichnis}

3-MRGN

4-MRGN

AWMF

BDA

$B D C$

BMG

COSMIN

COX

DGAI

DGCH

DGP

DRG

GG

IASP

ICB

ICD-10

M.

MRSA

N.

$\mathrm{Ncl}$

Ncll.

NRS

NSAR

OPS

PCA

Q

SOP

$\mathrm{StGB}$

TÜV

VRE

WDR
Gramnegative Stäbchen mit Resistenzen gegen 3 der 4 Antibiotikaleitsubstanzen

Gramnegative Stäbchen mit Resistenzen gegen alle 4 Antibiotikaleitsubstanzen

Arbeitsgemeinschaft der Wissenschaftlichen Medizinischen Fachgesellschaften e.V.

Berufsverband Deutscher Anästhesisten

Berufsverband Deutscher Chirurgen

Bundesministerium für Gesundheit

Consensus-based standards for the selection of health measurement instruments

Cyclooxygenase

Deutsche Gesellschaft für Anästhesiologie und Intensivmedizin

Deutsche Gesellschaft für Chirurgie

Deutsche Gesellschaft für Pneumologie und Beatmungsmedizin

Diagnosis-Related Groups

Grundgesetz

International Association for the Study of Pain

Intracranielle Blutung

International Statistical Classification of Diseases and Related Health Problems

Morbus

Methicilin-resistenter Staphylococcus aureus

Nervus

Nucleus

Nuclei

Numerische Ratingskala

Nichtsteroidale Antirheumatika

Operationen- und Prozedurenschlüssel

Patientenkontrollierte Analgesie

Quartil

Standard Operating Procedure

Strafgesetzbuch

Technischer Überwachungsverein

Vancomycin-resistente Enterokokken

Wide Dynamic Range 


\section{$1 \quad$ Einleitung}

Die International Association for the Study of Pain (IASP) definiert Schmerz als unangenehmes Sinnes- und Gefühlserlebnis, das mit aktueller oder potentieller Gewebeschädigung verknüpft ist oder mit Begriffen einer solchen Schädigung beschrieben wird (IASP Terminology 2020). Margo McCaffery beschreibt in ihrem Handbuch für die Pflegepraxis diese Wahrnehmung mit den Worten, „Schmerz ist das, was immer ein Patient darunter versteht, und Schmerz ist vorhanden, wann immer ein Patient ihn wahrnimmt" (McCaffery et al. 1997).

Physiologischerweise ist der Schmerz eine lebenswichtige Sinneswahrnehmung, die den Körper vor Gefahren und Verletzungen warnt (Schmidt et al. 2010; Benrath et al. 2012; Larsen 2013; Rothaug 2013). Diese subjektive Sinnesempfindung spiegelt aber nicht immer nur das Ausmaß einer Gewebeschädigung wider, sondern wird durch zusätzliche individuelle Faktoren wie Erwartungen und Emotionen beeinflusst (Meißner 2015; Larsen 2013).

Ursachen für ein akutes Schmerzempfinden können sehr vielfältig sein und müssen nicht zwangsläufig durch eine Operation oder ein Trauma ausgelöst werden, sondern können auch durch andere Erkrankungen (z. B. Herpes Zoster oder Migräne) induziert werden (Diener et al. 2011; Meißner 2015).

Der Schmerz kann sich aber auch von seiner physiologischen Warnfunktion entkoppeln und als eigenes Krankheitsbild auftreten. Neuroplastische Veränderungen, kortikale Lernprozesse wie klassische oder operante Konditionierung und vielfältige andere Chronifizierungsfaktoren können diese Dissoziation begünstigen (Schmidt et al. 2010). Als Folge kann man einen „akuten Schmerz" und einen „chronischen Schmerz" unterscheiden. Der „chronische Schmerz" besteht definitionsgemäß nach verschiedenen Quellen über einen Zeitraum von mindestens drei Monaten und verliert somit seine ursprüngliche physiologische Schutzfunktion. Die Deutsche Schmerzgesellschaft e. V. bezeichnet aktuell einen Schmerz als chronisch, wenn „dessen Dauer über das Ausmaß einer akuten Ursache hinaus nicht nachvollziehbar lange anhält" (Nobis et al. 2016).

Wird ein akutes Schmerzereignis nicht ausreichend behandelt, können zentrale neuroplastische Vorgänge begünstigt werden, die eine Sensibilisierung des Schmerzempfindens mit sich bringen und neben weiteren psychologischen, situativen und individuellen 
Faktoren die Entwicklung chronischer Schmerzen begünstigen (Nau 2010; Pogatzki-Zahn 2010; Schnabel und Pogatzki-Zahn 2010; Pogatzki-Zahn 2011; Woolf 2011).

Die anatomischen und physiologischen Zusammenhänge der Schmerzentstehung und Schmerzwahrnehmung sind sehr komplex. Die eigentliche subjektive Sinnesempfindung „Schmerz" entsteht letztlich erst durch die integrative Verarbeitung im Kortex (Schmidt et al. 2010). Da es in dieser Promotionsarbeit um die Entwicklung eines schmerztherapeutisch relevanten Qualitätssicherungsinstruments geht, werden diese komplexen Zusammenhänge nur kurz zur besseren Verständlichkeit erwähnt.

\subsection{Nozizeption - Schmerzwahrnehmung}

Die Nozizeption ist die Wahrnehmung von Reizen, die den Körper potenziell oder tatsächlich schädigen. Diese Reize werden von Nozizeptoren auf freien Nervenendigungen registriert und über afferente Schmerzfasern über das Rückenmark ins Gehirn geleitet.

Die Nozizeptoren kommen in großer Dichte vor allem in der Haut und nahezu in allen anderen Geweben des Körpers vor. Sie können meist polymodal oder rezeptorabhängig spezifisch auf mechanische, thermische und chemische Reize reagieren (Schmidt et al. 2010). Ihre Aktivierung führt dann entweder über ionotrope Mechanismen oder über metabotrope Mechanismen (z. B. durch Entzündungsmediatoren wie Prostaglandin-E2) zu einer Änderung der Membranleitfähigkeit und zu einer Depolarisation (Schmidt et al. 2010). Die Kenntnis über diese verschiedenen Aktivierungsmechanismen ist für eine optimale medikamentöse und physikalische Schmerztherapie von Bedeutung.

Im Allgemeinen können die nozizeptiven Nervenfasern entsprechend ihrer Myelinisierung und der daraus resultierenden Leitungsgeschwindigkeit klassifiziert werden. Für die Nozizeption sind hier vor allem die dünn myelinisierten Aठ-Fasern mit mittlerer Leitungsgeschwindigkeit und die kaum myelinisierten, langsam leitenden C-Fasern relevant. Entsprechend dieser unterschiedlichen Leitungsgeschwindigkeiten kommt es im unmittelbaren zeitlichen Verlauf zu unterschiedlichen Schmerzwahrnehmungen. A $\delta$-Fasern vermitteln einen früh wahrgenommenen Schmerz und C-Fasern einen eher späten Schmerz (Schmidt et al. 2010; Speckmann et al. 2013; Meißner 2015). Die Schmerzweiterleitung über diese nozizeptiven afferenten Bahnen benötigt wie jede somatosensorische Bahn drei Neurone. Das erste Neuron liegt im Spinalganglion und reicht mit dem peripheren 
Axonende in die Haut, Gewebe des muskuloskelettalen Systems und Organe. Über die Hinterwurzel gelangen die Fasern in das Hinterhorn des Rückenmarks.

Dort befindet sich in der Substantia gelatinosa, aber vor allem in den Laminae I und II das zweite Neuron (Trepel 2012). Zudem befinden sich in der Lamina V Wide-dynamic-rangeNeurone (WDR), die unterschiedliche somatosensorische Impulse (z. B. Schmerz oder aber auch Berührung) aus der Peripherie aufnehmen. Neben den nozizeptiven Sinnesimpulsen der A $\delta$ - und C-Fasern konvergieren hier auch die nicht nozizeptiven Impulse der myelinisierten, schnell leitenden $A \beta$-Fasern. Die $A \beta$-Fasern übertragen vor allem mechanische Reize niedriger Intensität wie Druck oder Berührung aus der Haut. Durch diese Konvergenz kann es jedoch dazu kommen, dass durch zentrale Sensibilisierung nicht nozizeptive Reize verstärkt oder sogar als schmerzhaft empfunden werden und Einfluss auf eine mögliche Chronifizierung nehmen können (Nau 2010; Woolf 2011; Speckmann et al. 2013; Bernateck et al. 2017).

Die Fasern kreuzen dann vom entsprechenden Neuron auf Segmentebene in der Comissura alba zur Gegenseite und verlaufen überwiegend über den Vorderseitenstrang (v. a. Tractus spinothalamicus lateralis) zum Thalamus (Nau 2010; Trepel 2012).

In den lateralen ( $\mathrm{Ncl}$. ventralis posterolateralis) und medialen Thalamuskernen (u. a. Ncll. intralaminares und centromedianus) befindet sich das dritte Neuron. Die Fasern der lateralen Kerne ziehen im Verlauf in den primären und sekundären somatosensorischen Kortex (Gyrus postcentralis). Hier findet vermutlich die sensorische und somatotopische Zuordnung der bewussten Schmerzwahrnehmung statt.

Die medialen Kerne sind vor allem mit dem Limbischen System verbunden, wodurch die Schmerzempfindung eine affektive Komponente erhält (Trepel 2012). Innerhalb des Gehirns besteht so eine sehr starke Vernetzung zwischen der Nozizeption, der emotionalen Empfindung und der kognitiven Komponente im präfrontalen Kortex. Diese engen Zusammenhänge wirken sich auch positiv wie negativ auf das resultierende individuelle Schmerzerleben aus. Insbesondere Wechselwirkungen zwischen dem subjektiven Schmerzerleben und den psychosozialen Einflüssen, spielen beim chronischen Schmerz eine immense Rolle (Nobis et al. 2016).

Im Allgemeinen ist der menschliche Körper sogar in der Lage, empfundene Schmerzen in gewissem Ausmaß selbst zu lindern. Neben den absteigenden hemmenden Nervenbahnen kommt hier auch den körpereigenen Opioidpeptiden (z. B. Enkephaline, Endorphine 
und Dynorphine) durch Bindung an die entsprechenden Opioidrezeptoren eine besondere Bedeutung zu. Durch sie wird eine Reizweiterleitung unterbunden, bzw. abgeschwächt (Schmidt et al. 2010; Trepel 2012; Larsen 2013; Meißner 2015). Die endogene Schmerzhemmung ist jedoch begrenzt und reicht in den meisten Fällen akuter Schmerzen nicht aus, um eine suffiziente Analgesie hervorzurufen, wodurch der Einsatz einer adäquaten Schmerztherapie meist notwendig bleibt. Eine mögliche Rolle spielt dafür auch eine Erschöpfung der körpereigenen Schmerzhemmung bei der Schmerzchronifizierung.

\subsection{Auswirkungen von Schmerzen auf den Heilungsverlauf}

Für schmerzleidende Patienten besteht daher nicht nur aus ethischen und rechtlichen Gründen (Berufsordnung der Bundesärztekammer (2015) § 1 Abs. 2; Grundgesetz (GG) Artikel 2 Abs. 2; Strafgesetzbuch (StGB) §§ 13, 223 Abs. 1, 229 und 323c) eine Verpflichtung, diese mit allen verfügbaren ärztlichen und pflegerischen Möglichkeiten fachgerecht zu lindern, sondern auch um den Heilungsverlauf zu fördern und die Entwicklung chronischer Schmerzen möglichst zu verhindern (Kutzer 2008; Meissner et al. 2008; Diener et al. 2011; Korczak et al. 2013; Larsen 2013; Gerbershagen et al. 2014; Meißner 2015; Nobis et al. 2016). So kann eine konsequente Schmerztherapie sowohl die Patientenzufriedenheit steigern als auch die stationäre Verweildauer verkürzen und die schmerzbedingte Morbidität und Mortalität reduzieren. Eine konsequente Schmerztherapie kann im Kontext des DRG-Systems auch potentiell positive wirtschaftliche Auswirkungen für entsprechend engagierte Kliniken bedeuten.

Neben einer möglichen Chronifizierung können Schmerzen grundsätzlich Funktionsstörungen in nahezu allen Organsystemen hervorrufen (Larsen 2013; Heck et al. 2017).

Somatische Komplikationen können u. a. durch schmerzbedingte eingeschränkte Bewegungen oder reduzierte Motivation begünstigt werden. So steigen beispielsweise die Risiken eine Thrombose (begünstigt durch die Virchow'sche Trias) oder einen Dekubitus zu entwickeln deutlich an.

Schmerzbedingte respiratorische Störungen können durch eingeschränkte Atemarbeit (z. B. Schonatmung) zu Atelektasen und so zu einem gestörten Gasaustausch führen. Die 
Folge wäre eine Begünstigung von Infektionen und einer Pneumonie. Je nach Allgemeinzustand und Morbidität erhöht eine nosokomial erworbene Pneumonie die Letalität und die Behandlungsdauer eines Patienten (Larsen et al. 2016; DGP 2017; Heck et al. 2017). Schwere Erkrankungen und große operative Eingriffe führen grundsätzlich zu einer gesteigerten Stressreaktion und Cortisolausschüttung. Diese Stressreaktion wird durch Schmerzen zusätzlich verstärkt, wodurch gleichzeitig die Wundheilung beeinträchtigt und das Infektionsrisiko steigen kann. Sehr starke Schmerzen führen über Aktivierung des Sympathikotonus zu einer Belastung des kardiovaskulären Systems mit Tachykardie, Hypertonie und peripherer Vasokonstriktion. Die Folgen sind eine Zunahme der Herzarbeit und ein deutlich gesteigerter myokardialer Sauerstoffverbrauch (Larsen 2013). Vor allem ältere und bereits kardial vorerkrankte Patienten können darunter zunehmend belastet werden.

Der Gastrointestinaltrakt kann auf Schmerzen mit unangenehmen vegetativen Begleitsymptomen wie Übelkeit und Erbrechen reagieren. Zusammen mit einer starken Stressreaktion kann so auch das Risiko einer Ulzeration im Gastrointestinaltrakt (z. B. Ulcus ventriculi oder Ulcus duodeni) ansteigen, die wiederum gleichzeitig mit einem möglichen Blutungs- und Perforationsrisiko einhergeht (Bornemann und Gaber 2013; Striebel 2017). Die Überaktivität des Sympathikus beeinträchtigt dazu die Motilität, die im schlimmsten Fall sogar zu einem paralytischen lleus führen kann (Larsen 2013), der mit weiteren möglichen Komplikationen und einer längeren Krankenhausverweildauer einhergeht.

Neben der Gefahr solcher somatischen Komplikationen, können durch die bereits genannte komplexe Entstehung und Beeinflussung des Schmerzerlebens das seelische Wohlbefinden und die gesamte Zufriedenheit des Patienten eingeschränkt werden. Inadäquat schmerztherapierte Patienten können so unter einer eingeschränkten Lebensqualität leiden und z. B. zu Schlafstörungen oder depressiven Verstimmungen neigen (Schnabel und Pogatzki-Zahn 2010; Menche und Asmussen-Clausen 2011; Nobis et al. 2016). Zudem kann so auch in der Folge die Compliance dieser schmerzleidenden Patienten mit dem therapeutischen Team negativ beeinträchtigt werden (Menche und Asmussen-Clausen 2011; Nobis et al. 2016). Vor allem für länger anhaltende Schmerzen besteht eine potentiell sehr komplexe Vernetzung von Komorbiditäten auf somatischer und psychischer Ebene. 
Die Entwicklung chronischer Schmerzen gilt nach aktueller wissenschaftlicher Meinung als ein multifaktorielles Geschehen. Neben einigen individuellen und unbeeinflussbaren Faktoren wie dem Alter oder dem Geschlecht, besteht beispielsweise durch anhaltende akute Schmerzen die Gefahr der Entstehung eines chronischen postoperativen Schmerzes. Auch bereits präoperativ bestehende Schmerzen können eine Chronifizierung vermutlich auf Basis einer vorab eingetretenen Sensibilisierung und neuronaler Plastizität begünstigen. (Nau 2010; Pogatzki-Zahn 2010; Schnabel und Pogatzki-Zahn 2010; Pogatzki-Zahn 2011; Gerbershagen et al. 2014). Der Heilungsverlauf kann daher bereits präoperativ sowohl positiv wie negativ durch adäquate oder mangelhafte Schmerztherapie beeinflusst werden. Um solche schmerzbedingten und schmerzassoziierten, teils schwerwiegenden Komplikationen zu reduzieren und die dafür angemessenen ärztlichen und pflegerischen Prophylaxen zu ermöglichen, ist es notwendig eine optimale subjektive Schmerzerfassung am Patientenbett und eine dafür angemessene Schmerztherapie durchführen zu können.

\subsection{Stand der Forschung zur Qualität der Schmerztherapie}

Grundsätzlich sollten heute in den meisten Krankenhäusern adäquate Möglichkeiten für eine ausreichende Schmerztherapie vorhanden sein und dennoch beklagen viele Patienten postoperativ oder im Rahmen einer konservativen Behandlung starke und nicht akzeptable Schmerzen. Ausgehend von dieser Beobachtung dazu wurde im Jahr 2003 das Projekt „Schmerzfreies Krankenhaus“ gestartet, mit dem bundesweit das Schmerzmanagement verbessert werden soll und mittlerweile von verschiedenen Initiativen der Zertifizierung, wie von Certkom oder dem TÜV weiterentwickelt wurde (Maier et al. 2010; Böhmer et al. 2012; Maier et al. 2013).

In einer großen anonymisierten Datenerhebung im Rahmen einer Zertifizierung im Zeitraum von 2004 bis 2006 konnte gezeigt werden, dass mehr als 50\% der behandelten Patienten über Schmerzen unter Belastung klagten. Ebenfalls waren mehr als die Hälfte dieser Patienten sehr unzufrieden mit der erhaltenen Schmerztherapie (Maier et al. 2010). In dieser Studie konnte auch gezeigt werden, dass der Anteil nicht ausreichend wirksamer Schmerzbehandlungen bei konservativ behandelten Patienten signifikant höher war als nach Operationen (Maier et al. 2010). 


\subsubsection{Qualitätsmanagement und Benchmarking}

Um solche Ungleichgewichte besser erfassen und minimieren zu können finden auch bereits zunehmend Qualitätssicherungsinstrumente im klinischen Alltag Anwendung. Diese sind beispielsweise die Anwendung von einfachen Mitteln wie der numerischen Ratingskala (NRS) zur Schmerzmessung, klinikinterne Verfahrensanweisungen (SOP) zum Umgang mit schmerzleidenden Patienten oder der Einsatz von speziellen Techniken und weitergebildeten Ärzten und Pflegekräften im Rahmen eines Akutschmerzdienstes (Meißner 2015; Erlenwein 2016). Auf der Homepage der Arbeitsgemeinschaft der Wissenschaftlichen Medizinischen Fachgesellschaften e. V. (AWMF) sind zudem alle klinikübergreifenden Leitlinien veröffentlicht und frei einsehbar.

Die Arbeitsgruppe um Winfried Meissner aus Jena reagierte bereits ebenfalls im Jahr 2003 auf die unklare bis unzureichende Versorgungssituation und entwickelte das Benchmarking-Projekt QUIPS zur besseren Erfassung der Qualität der Akutschmerztherapie bei postoperativen Patienten. QUIPS steht für „Qualitätsverbesserung in der postoperativen Schmerztherapie" und bietet dem Anwender ein umfangreiches, aber auch kostenpflichtiges Qualitätssicherungsinstrument basierend auf der Idee des Benchmarking und nicht der Zertifizierung (Meissner et al. 2006; Meissner et al. 2008).

Der Begriff Benchmarking stammt ursprünglich aus der Wirtschaft und ist eine Möglichkeit zur Wettbewerbsanalyse und Qualitätsverbesserung. Es wird nun auch zunehmend im Gesundheitswesen angewendet, um bestehende Unterschiede mit den dafür möglicherweise vorliegenden Gründen zu analysieren und geeignete Verbesserungsmöglichkeiten anhand der besten Ergebnisse herauszufinden. Innerhalb dieser Analysen kann zwischen einem internen (klinikintern auch als interventionsbezogene Vor- und Nachbeobachtung) und externen Benchmarking (zwischen verschiedenen Krankenhäusern) unterschieden werden (Ettorchi-Tardy et al. 2012; Meißner 2016; Gabler Wirtschaftslexikon - Benchmarking 2017). Auf der Homepage von QUIPS wird das Projektziel formuliert als „Verbesserung der Ergebnisqualität in der postoperativen Schmerztherapie durch eine standardisierte Erhebung weniger Qualitätsindikatoren, ihre Analyse und Rückmeldung an die beteiligten Kliniken. Ein webbasiertes automatisiertes Feedback ermöglicht internes und externes Benchmarking sowie eine kontinuierliche Verlaufsbeobachtung". 
Den teilnehmenden Kliniken ist es mit QUIPS möglich, ihre erhobenen Daten selbstständig in die Benchmarking-Datei einzugeben und eine direkte Analyse zu erhalten. Gleichzeitig können so auch die Maßnahmen anderer Kliniken anonymisiert eingesehen werden.

Dieses Projekt wurde in der anfänglichen Förderphase von 2003 bis 2006 umfangreich vom Bundesministerium für Gesundheit (BMG) unterstützt und steht seit 2005 unter der Schirmherrschaft der Deutschen Gesellschaft für Anästhesiologie und Intensivmedizin (DGAI) und dem Berufsverband Deutscher Anästhesisten (BDA) und finanziert sich seitdem aus den Beiträgen der Kliniken. Die Deutsche Gesellschaft für Chirurgie (DGCH) und der Berufsverband Deutscher Chirurgen (BDC) kooperieren seit 2007 ebenfalls mit dem Projekt. Mit derzeit mehr als 200 teilnehmenden Kliniken und bereits über einer halben Million an Datensätzen ist es das weltweit größte Akutschmerzregister (Stand September 2017, QUIPS-Homepage 2017).

Diese Erhebung trägt die Ergebnisse der Gesamtqualität der Versorgung mithilfe eines Fragebogens einerseits aus Patientensicht zusammen, andererseits ergänzt durch fallbezogene Parameter zu Prozessen und eingeschränkt zu Strukturen. Die Ergebnis- und die Prozessparameter können daraufhin zum spezifischen Vergleich der jeweiligen Erkrankungen und der operativen Eingriffe/Prozeduren genutzt werden (Meissner et al. 2008; Gerbershagen et al. 2014).

Als internationales Pendant zu QUIPS wurde im Zeitraum von 2009 bis 2012 das Projekt „Improvement in postoperative PAIN OUTcome“ von der Europäischen Union (EU) gefördert. PAIN OUT basiert u.a. auf den Erfahrungen mit QUIPS und wird mehrsprachig an 60 Kliniken in 17 Staaten weltweit eingesetzt (Stand 2014). Zusätzlich zum internen und externen Benchmarking bietet PAIN OUT noch zwei weitere Möglichkeiten zur Qualitätssicherung an. Zum einen wird für die Teilnehmer in der „Electronic Knowledge Library" eine Übersicht über relevante Leitlinien zur postoperativen Akutschmertherapie bereitgestellt und zum anderen kann ein fallbasiertes Auswertungssystem bei individuellen Therapieentscheidungen weiterhelfen („Clinical Decision Support System“). Die Koordination des Projektes erfolgt analog zu QUIPS durch das Universitätsklinikum in Jena (Rothaug et al. 2013; Zaslansky et al. 2014; Zaslansky et al. 2015). Aufgrund dieser vielen Gemeinsamkeiten wurden im Jahr 2016 QUIPS und PAIN OUT zusammengeführt, um dem Anwender 
so nicht nur eine nationale, sondern auch eine internationale Vergleichsplattform anbieten zu können (Meißner 2016).

\subsection{Ziel dieser Promotionsarbeit}

Die Prävalenz von vorbestehenden chronischen Schmerzen variiert je nach Fachrichtung im Bereich zwischen 46\% und 75\%, bei über 65-jährigen Patienten liegt diese sogar zwischen 67\% und 80\% (Kindler und Maier 2008). Besonders diese Patientengruppe macht einen Großteil der Patienten auf nicht operativen Stationen aus und wird durch den demographischen Wandel in Zukunft weiter zunehmen. In der Folge werden somit auch chronische Schmerzen als Komorbidität im klinischen Alltag weiter ansteigen (PogatzkiZahn et al. 2008; Pogatzki-Zahn 2011; Maier et al. 2013).

Zudem bemängeln viele ärztliche und pflegerische Kollegen, trotz der zahlreichen bereits vorhandenen Qualitätssicherungsinstrumente neben Defiziten in der schmerzspezifischen Aus- und Fortbildung, unklare Zuständigkeiten oder mangelnde Therapiekonzepte. Dazu komme das Fehlen einfach anwendbarer Mittel, um den subjektiven Bedarf und die Qualität einer angewendeten Schmerztherapie aus Patientensicht sicher erfassen und decken zu können (Nestler und Osterbrink 2009; Nestler et al. 2013).

Da sich das QUIPS-Projekt und PAIN OUT rein auf die postoperativen Patienten bezieht, besteht auch hier weiterhin der Mangel, dass konservativ behandelte Patienten in der klinischen Forschung in diesem Zusammenhang unterrepräsentiert sind (Maier et al. 2010; Maier et al. 2013). Es wurde daher als Projektmodul QUIKS (Qualitätsverbesserung in der konservativen (nicht operativen) Schmerztherapie) entwickelt.

Das Ziel des QUIKS-Moduls soll sein, ein vergleichbares und leicht anwendbares Benchmarking-Tool für die Qualitätssicherung im klinischen Alltag konservativer (nicht operierter) Patienten zu gestalten. Das Prinzip und die Gestaltung des QUIKS-Fragebogens basieren weitgehend, bis auf wenige Anpassungen an nicht operative Patienten, auf dem von QUIPS. Der deutschsprachige Fragebogen gliedert sich daher auch in die Bereiche Schmerzintensität, funktionelle Beeinträchtigung, therapiebedingte Nebenwirkungen und die subjektive Zufriedenheit mit der Therapie durch den Patienten. Langfristig gesehen ist ebenfalls eine Verschmelzung beider Projekte vorgesehen. 
Die ersten Maßnahmen zur Entwicklung von QUIKS wurden bereits im Vorfeld zu dieser Promotionsarbeit im Sinne einer ersten Machbarkeitsstudie eingeleitet. Im Rahmen dieser Promotionsarbeit soll nun eine erste quantitative und qualitative Auswertung vorgenommen werden, um danach QUIKS in die tägliche klinische Praxis zu übernehmen. Da das aktuelle Befragungsmodell nur eine einmalige und anonymisierte Erfassung vorsieht und aufgrund der stattfindenden Begleittherapie eine zweite Befragung methodisch fraglich ist, ist eine Validierung im engeren Sinne (z. B. durch Test-Retest-Validität) zum aktuellen Zeitpunkt dieser Untersuchung nicht umsetzbar und sollte ggfs. in zukünftigen Projekten angestrebt werden.

In insgesamt vier Phasen werden die Inhalte des Fragebogens bzw. innerhalb der Prozessparameter an einer größeren Patientengruppe in drei verschiedenen Kliniken getestet (Tabelle 1). Die erste Testphase fand als Voruntersuchung an 114 Patienten an der Universitätsmedizin Göttingen und dem Krankenhaus Martha-Maria in Halle vor dieser Promotionsarbeit statt. Die Ergebnisse dieser Machbarkeitsstudie dürfen mit freundlicher Genehmigung der Klinik für Schmerzmedizin unter der Leitung von Professor Dr. Frank Petzke an der Universitätsmedizin Göttingen in dieser Promotionsarbeit verwendet werden.

Hier wurde der Inhalt des Ergebnisfragebogens auf Vollständigkeit und Verständlichkeit geprüft. Die in dieser Zeit befragten Patienten waren alle in einer Fachdisziplin der nicht operativen Medizin stationär in die Klinik aufgenommen worden. In dieser Voruntersuchung wurden Formulierungen einzelner Fragen überarbeitet und es wurden Schwierigkeiten mit der praktischen Durchführung, bezüglich eines einheitlichen Befragungstags und der Diagnoseerfassung zwischen den beiden Kliniken deutlich, da zum Beispiel eine konkrete ICD-10-Kodierung nicht bei allen Patienten am Befragungstag vorlag. Eine einheitliche, standardisierte Erfassung der jeweiligen Hauptdiagnose mithilfe der ICD-10-Kodierung, erschien somit im Vergleich zur OPS-Kodierung in QUIPS als nicht geeignet.

Ausgehend von den Erkenntnissen der Vorerhebung wurden die weiteren Testphasen dann innerhalb dieser Promotionsarbeit bearbeitet. Zielsetzungen waren in der ersten Phase innerhalb der Prozessparameter eine von der ICD-10-Kodierung unabhängige Wortdiagnoseliste und eine erste Medikamentenauswahl zu entwerfen und abschließend eine großflächigere Testung durchzuführen. 
Ein Ziel der Promotionsarbeit war somit Diagnosen und Medikamente, die besonders häufig vorkommen bzw. welche selten bis nie erfasst werden, für die jeweilige Dokumentation vor der klinischen Einführung zu prüfen und noch einmal anzupassen. So sollte geprüft werden, ob bei konservativ behandelten Patienten eine Wortdiagnoseliste als Benchmarking-Faktor grundsätzlich einsetzbar ist, oder ob aufgrund einer zu starken Heterogenität der klinischen Situation eine andere Herangehensweise angestrebt werden sollte.

Ein weiteres Ziel war den Ergebnisfragebogen selbst, vor allem in Bezug auf die Verständlichkeit der einzelnen Fragen und die inhaltliche Konsistenz zu prüfen, um auch hier möglicherweise Optimierungen umsetzen zu können. Mögliche Beispiele hierfür werden im Ergebnisteil dieser Arbeit aufgezeigt.

Zusätzlich wurden Teilaspekte einer formalen Validierung (z. B. Faktorenanalyse) analog zur Vorarbeit zum QUIPS-Modul für postoperative Patienten durchgeführt und die Ergebnisse mit QUIPS verglichen.

Nach Abschluss der Projektphasen werden alle am Projekt beteiligten Fachkräfte der teilnehmenden Kliniken zur praktischen Umsetzung von QUIKS befragt. Es soll besonders von den späteren, klinischen Anwendern ein breites Spektrum an Feedback zur Anwendbarkeit eingeholt werden, um ein möglichst optimales Projektergebnis erzielen zu können und Anweisungen für eine vergleichbare Durchführung der Erhebung in einem zukünftigen Benchmark-Prozess sicher zu stellen. 


\section{Material und Methoden}

Das Befragungsmodell basiert auf der Grundlage des QUIPS-Projekts zum Benchmarking in der postoperativen Schmerztherapie. Als passendes Modul dazu wurde der QUIKS-Fragebogen gestaltet, um auch die konservativen (nicht operativen) Patienten befragen zu können. Durch diesen standardisierten Fragebogen können so die Ergebnis- und Prozessparameter optimal erfasst und später mit der QUIPS-Datenbank verbunden werden. Beim Fragebogen- und Studiendesign wurde daher auch vor allem auf die Methodik der Analysen zum QUIPS-Fragebogen von Meißner et al. (2008) und von Rothaug et al. (2013) zurückgegriffen, die sich umfangreich mit der Gestaltung und Validierung von QUIPS und PAIN OUT befasst haben. Zum einfacheren Vergleich mit QUIKS befinden sich die jeweils aktuellsten Versionen von QUIPS und PAIN OUT im Anhang (6.1, 6.2 und 6.3) dieser Arbeit.

Tabelle 1: Übersicht zum Projektablauf von QUIKS

\begin{tabular}{|c|l|c|l|}
\hline Phase & Bezug zur Promotionsarbeit & Anzahl & Schwerpunkte \\
\hline 1 & $\begin{array}{l}\text { Vorab durch Universität } \\
\text { Göttingen }\end{array}$ & 114 & $\begin{array}{l}\text { Testung des Fragebogens auf Vollständigkeit und Verständlich- } \\
\text { keit in Göttingen und Halle }\end{array}$ \\
\cline { 1 - 1 } 2 & $\begin{array}{l}\text { Bestandteile der vorliegen- } \\
\text { den Promotionsarbeit }\end{array}$ & 42 & $\begin{array}{l}\text { Entwicklung der Prozessparameter: Demographische Parame- } \\
\text { ter, Wortdiagnoseliste und Medikamentenauswahl }\end{array}$ \\
\cline { 1 - 1 } 3 & 30 & Ergänzung zum Fragebogen: Tabelle mit Schmerzentitäten \\
& & 1317 & $\begin{array}{l}\text { Hauptprojektphase: Keine inhaltlichen Änderungen mehr. Groß- } \\
\text { flächige Testung an den Kliniken in Göttingen, Jena und Halle }\end{array}$ \\
\hline
\end{tabular}

\subsection{Projektablauf}

Mit Beginn dieser Promotionsarbeit wurde auf Grundlage der Erkenntnisse aus der Voruntersuchung die zweite Testphase im März 2016 an 42 Patienten in Göttingen durchgeführt. Ziel dieser Testphase war die Erstellung und Erprobung der zum Ergebnisfragebogen entwickelten Prozessparameter und deren Überprüfung auf Vollständigkeit, insbesondere der neu entwickelten Wortdiagnoseliste, an einem zunächst kleinen Patientenkollektiv. In dieser Erprobungsphase der Promotionsarbeit galt es auch Schwierigkeiten in der Dokumentation ausfindig zu machen, um die abschließende Projektphase vorzu- 
bereiten. Eines dieser Probleme zeigte sich bei der Dokumentation der Krankenhausverweildauer der Patienten bereits während der Voruntersuchung. Um ein validierbares Ergebnis zu erhalten, wurde ab dieser Phase der dritte Krankenhaustag als einheitlicher Befragungstag festgelegt.

Aus der zweiten Testphase entstand die Idee zu erfragen, welche Schmerzentitäten den jeweiligen Patienten während des Krankenhausaufenthalts am schlimmsten beeinträchtigen. Ein ähnliches Vorgehen war bereits in der Zertifizierungsstrategie von Certkom e. V. aufgenommen worden. Davon ausgehend wurde eine tabellarische Auswahl verschiedener Schmerzentitäten in den Ergebnisfragebogen aufgenommen. In einer sehr kurzen, abschließenden dritten Testphase wurde in Göttingen an 30 Patienten die neu eingefügte Tabelle zur Differenzierung der empfundenen Schmerzentitäten getestet. Die Prozessparameter wurden in diesem abschließenden Zeitraum nicht miterfasst.

Die Hauptprojektphase der Patientenbefragungen erfolgte danach im Zeitraum von April bis Oktober 2016. In dieser Zeit wurden auf den Stationen insgesamt 1317 konsekutiv geeignete Patienten ( $\mathrm{n}=$ Anzahl) befragt, die sich auf die Universitätsmedizin Göttingen ( $n=1050)$, das Universitätsklinikum Jena $(n=149)$ und das Krankenhaus Martha-Maria in Halle $(n=118)$ aufteilten.

Die Befragungen in Jena und Halle wurden für die Projektphase, entsprechend einer schriftlichen Verfahrensanweisung, von den dort beschäftigten algesiologischen Fachassistenten übernommen. So konnte zum einen die Stichprobengröße erweitert werden und zum anderen wurde gleichzeitig die allgemeine Anwendbarkeit des Fragebogens durch projektferne Fachkräfte getestet. Alle beteiligten ärztlichen und nicht ärztlichen Personen unterlagen standortübergreifend der Schweigepflicht.

Die Befragungen wurden an diesen Kliniken bei Patienten der konservativen (nicht operativen) Disziplinen der Inneren Medizin, Neurologie und Dermatologie durchgeführt. Zur Inneren Medizin zählen hier die Kardiologie, Pulmologie, Gastroenterologie, Hämatologie/Onkologie, Nephrologie und Rheumatologie. Die Neurologie teilte sich am Befragungsort Göttingen entsprechend der dortigen Spezialisierung in die Neurologie und die Klinische Neurophysiologie auf. 
Als validierbare Selektionsfaktoren wurden für diese Patienten die Volljährigkeit und die Durchführung der Befragung am dritten Krankenhaustag festgelegt. Der Tag der Aufnahme wurde dabei einheitlich als Tag 0 gewertet. Eine vorherige Bewertung oder Selektion entsprechend der Aufnahmediagnose oder einer bereits bestehenden chronischen Schmerzerkrankung wurde für die Teilnahme an der Befragung nicht durchgeführt.

Für den Standort Göttingen erfolgte die tägliche Selektion der zu befragenden Patienten mittels Bettenbelegungsplänen aus den jeweiligen Kliniken (z. B. Case Management) und über das klinikinterne Computerprogramm ixserv.4. In Jena und Halle wurden die Patienten nach Akteneinsicht auf der jeweiligen Station ausgewählt.

Tabelle 2: Übersicht über die Teilnahmekriterien

\begin{tabular}{|c|c|c|}
\hline Einschlusskriterien & $\begin{array}{l}\text { - } \quad \text { Einwilligung durch den Patienten } \\
\text { - } \quad \text { Volljährigkeit } \\
\text { - } \quad \text { Krankenhaustag } 3(\text { Aufnahme = Tag 0) } \\
\text { - } \quad \text { Aufnahme in eine nicht operative Fachabteilung }\end{array}$ & $n=973$ \\
\hline Ausschlusskriterien & $\begin{array}{l}\text { - Ablehnung durch den Patienten } \\
\text { - Abweichungen vom definierten Aufnahmetag } \\
\text { - Abwesenheit oder Entlassung } \\
\text { - Gesundheitliche Gründe } \\
\text { - Sprachbarrieren }\end{array}$ & $n=344$ \\
\hline
\end{tabular}

In der allgemeinen praktischen Durchführung wurde nach einer kurzen Projektvorstellung von den jeweilig selektierten Patienten am dritten Aufnahmetag das Einverständnis eingeholt, dass sie befragt und ihre Daten anonymisiert erhoben werden durften. Eine erneute Befragung des Patienten im Verlauf war nicht vorgesehen. Die Patienten wurden darauf hingewiesen, dass die Teilnahme freiwillig war und jederzeit von Ihnen beendet werden konnte ohne Auswirkungen auf ihre klinische Versorgung und weitere Behandlung.

Während der Befragung wurden keine persönlichen Daten, wie Name, Geburtsdatum oder die Adresse schriftlich erfasst. Es bestand hier nur ein kurzer Moment, in dem der Befragende auf der Station während der Dokumenteneinsicht einen direkten Bezug vom Patienten zu dessen spezifischen Daten besaß. Nach der anonymisierten Eingabe in die 
Benchmarking-Datei war keine Rückverfolgung mehr von den Daten zum jeweiligen Patienten möglich. Ein Widerruf der Zustimmung zur Nutzung der Daten war aufgrund dieser anonymisierten Eingabe zu einem späteren Zeitpunkt ebenfalls nicht mehr möglich. Der Patient erklärte sich damit schriftlich einverstanden oder hatte nach der Aufklärung die Möglichkeit eine Erhebung abzulehnen.

Diese Bestätigung wurde separat vom Fragebogen zur Vollständigkeit der Studie abgeheftet, ohne dass hierdurch nachträglich ein Zusammenhang von der jeweiligen Einwilligung zum ausgefüllten Fragebogen hergestellt werden konnte (keine Pseudonymisierung). Sofern eine Einwilligung erfolgte, wurde dem Patienten ein Ergebnisfragebogen ausgeteilt, dem zuvor numerisch ein entsprechender Prozessparameterbogen zugeordnet wurde.

Nach einer kurzen Instruktion durch den Befragenden wurde den Patienten ausreichend Zeit eingeräumt, um den Fragebogen selbstständig auszufüllen. Der Zeitaufwand für den Patienten betrug in der Regel etwa 5 bis 10 Minuten.

Alternativ bestand auch die Möglichkeit, dass die Befragung in Form eines Interviews durchgeführt wurde, sofern der Patient dies wünschte oder gesundheitlich nicht in der Lage war, den Bogen selbst auszufüllen. In so einem Fall wurde aufgrund der Erfahrungen im Projekt von einem deutlich längeren und individuell variierenden zeitlichen Rahmen pro Patienten ausgegangen.

\subsection{Ergebnisfragebogen}

Der sich in der Entwicklung an QUIPS orientierende und ausgehändigte Ergebnisfrageboden umfasst insgesamt zehn Fragen, die sich zum Teil aus mehreren Teilfragen zusammensetzen. Die Beantwortung der einzelnen Fragen war entweder mithilfe einer 11-stufigen NRS oder in binärer Form durch „ja oder nein“ möglich. Die erste umfangreiche Testung des Ergebnisfragebogens wurde schon vor dieser Promotionsarbeit durchgeführt. Hierbei wurde insbesondere auf die allgemeine Anwendbarkeit und Verständnisschwierigkeiten in der Praxis geachtet (Tabelle 1). In den sich anschließenden zwei Testphasen wurde der Fragebogen um die Schmerzentitätentabelle ergänzt und bis zur endgültigen Fassung für die Hauptprojektphase weiter getestet.

In der Folge werden die einzelnen Fragen aus der endgültigen Fragebogenversion im Detail erläutert. 


\subsubsection{Chronisches Schmerzleiden}

Im Ergebnisfragebogen selbst stellt die erste Frage eine abhängige Frage dar (Abbildung 1). Nur wenn der Patient Frage 1a mit „ja“ beantwortet, werden die beiden folgenden Fragen relevant.

Zu Beginn (Frage 1a) wurde zunächst gefragt, ob beim Patienten vor der aktuellen Klinikeinweisung bereits ein chronischer Schmerz vorlag, definiert als ständig über einen Zeitraum von mindestens drei Monaten.

Wenn die Patienten im Vorfeld bereits einen chronischen Schmerz hatten, sollten sie eine Aussage über die typische Schmerzintensität (Frage 1b) mittels NRS machen und beurteilen, ob sie aktuell wegen dieser Schmerzen oder aus einem anderen Grund in die Klinik aufgenommen (Frage 1c) worden sind.

1a. Hatten Sie ständige Schmerzen, die drei Monate oder länger andauerten, bevor Sie ins Krankenhaus gekommen sind?

口 Ja a Nein

1b. Falls ja, wie stark waren diese Schmerzen meistens?

\begin{tabular}{l|l|l|l|l|l|l|l|l|c|c|}
\hline $\mathbf{0}$ & $\mathbf{1}$ & $\mathbf{2}$ & $\mathbf{3}$ & $\mathbf{4}$ & $\mathbf{5}$ & $\mathbf{6}$ & $\mathbf{7}$ & $\mathbf{8}$ & $\mathbf{9}$ & $\mathbf{1 0}$ \\
\hline $\begin{array}{l}\text { Kein } \\
\text { Schmerz }\end{array}$ & \multicolumn{10}{|c|}{$\begin{array}{r}\text { Stärkster } \\
\text { vorstellbarer Schmerz }\end{array}$}
\end{tabular}

1c. Falls ja, waren diese Schmerzen Grund für die Krankenhausaufnahme?

$\square$ ja $\square$ nein $\square$ weiß nicht

Abbildung 1: Frage zu vorbestehenden chronischen Schmerzen mit drei abhängigen Teilfragen

\subsubsection{Schmerzentitäten und Schmerzintensitäten}

In einer tabellarischen Übersicht über verschiedenste Schmerzentitäten (Abbildung 2) konnten die Patienten ihre möglicherweise in der Klinik empfunden Schmerzen, auch als Mehrfacheingabe, eingruppieren. Zusätzlich wurden sie aufgefordert von diesen empfundenen Schmerzen den für sie wichtigsten oder „schlimmsten Schmerz" festzulegen. Diese neue Tabelle wurde nach dem Vorbild der Vorerhebungen zur Zertifizierung von Certkom weiter modifiziert und findet sich nicht in QUIPS, da die jeweilige Schmerzursache bei QUIPS in Bezug zur konkreten OP gemessen wird.

In den darauffolgenden Teilfragen von den Fragen 2 und 3 wurde mittels NRS erfragt, wie stark der Patient am Aufnahmetag, während Untersuchungen bzw. Behandlungen, und innerhalb der vergangenen 24 Stunden in Ruhe, bei Belastung und als Spitzenwert Schmerzen empfunden hat (Abbildung 3). Diese Fragen weichen in ihrer Form nicht 
grundsätzlich von QUIPS ab, neu jedoch ist der Bezug auf Schmerzen nach Prozeduren oder Maßnahmen.

2a. Haben Sie bisher während Ihres Aufenthaltes im Krankenhaus an einer oder mehreren der folgenden Schmerzarten gelitten?

\begin{tabular}{|l|c|c|}
\hline $\begin{array}{c}\text { Bitte kreuzen Sie in der ersten Spalte die Kästchen der Schmerzarten an, die bei } \\
\text { Ihnen zutreffen. Geben Sie bitte auch die Schmerzen an, die eventuell in keinem } \\
\text { Zusammenhang mit ihrer aktuellen Erkrankung stehen. Dann kreuzen Sie in der } \\
\text { zweiten Spalte an, welche der Schmerzarten für Sie am schlimmsten war/ist. }\end{array}$ & $\begin{array}{c}\text { Ja } \\
\text { (hier alles } \\
\text { ankreuzen, } \\
\text { was bei Ihnen } \\
\text { zutrift) }\end{array}$ & $\begin{array}{c}\text { Der } \\
\text { schlimmste } \\
\text { Schmerz } \\
\text { (hier bitte nur } \\
\text { eines } \\
\text { ankreuzen!) }\end{array}$ \\
\hline Gesichtsschmerzen & $\square$ & $\square$ \\
\hline Kopfschmerzen & $\square$ & $\square$ \\
\hline Nackenschmerzen & $\square$ & $\square$ \\
\hline Rücken- Oder Kreuzschmerzen & $\square$ & $\square$ \\
\hline Mund-/ Halsschmerzen (z.B. beim Schlucken/Essen) & $\square$ & $\square$ \\
\hline Herzschmerzen (z.B. Angina pectoris) & $\square$ & $\square$ \\
\hline Schmerzen im Brustkorb (z.B. beim Husten, Atmen) & $\square$ & $\square$ \\
\hline Bauchschmerzen & $\square$ & $\square$ \\
\hline Leistenschmerzen & $\square$ & $\square$ \\
\hline Blasen- Oder Nierenschmerzen & $\square$ & $\square$ \\
\hline Gelenk- Oder Knochenschmerzen & $\square$ & $\square$ \\
\hline Muskelschmerzen & $\square$ & $\square$ \\
\hline Nervenschmerzen (z.B. Ischias, Nervenverletzungen oder & $\square$ & $\square$ \\
\hline Polyneuropathie bei Zuckerkrankheit, Gürtelrose) & $\square$ & $\square$ \\
\hline Schmerzen bei Durchblutungsstörungen & $\square$ & $\square$ \\
\hline Wund- oder Narbenschmerzen & $\square$ & $\square$ \\
\hline Schmerz bei Untersuchung, Eingriff, Punktion, Behandlung & $\square$ & $\square$ \\
\hline Andere Schmerzen (bitte benennen): & $\square$ & $\square$ \\
\hline
\end{tabular}

Abbildung 2: Frage 2a und die Liste der möglichen Schmerzentitäten 
2b. Wie stark waren Ihre Schmerzen bei der Aufnahme ins Krankenhaus?

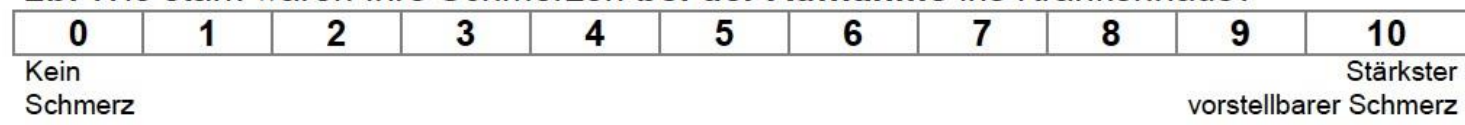

2c. Bitte kreuzen Sie hier den stärksten Schmerz an, den Sie seit Ihrem Krankenhausaufenthalt bei schmerzhaften Prozeduren/Maßnahmen (z.B. Untersuchung, Eingriff, Punktion, Behandlung) hatten.

\begin{tabular}{|l|l|l|l|l|l|l|l|l|l|l|}
\hline $\mathbf{0}$ & $\mathbf{1}$ & $\mathbf{2}$ & $\mathbf{3}$ & $\mathbf{4}$ & $\mathbf{5}$ & $\mathbf{6}$ & $\mathbf{7}$ & $\mathbf{8}$ & $\mathbf{9}$ & $\mathbf{1 0}$ \\
\hline $\begin{array}{l}\text { Kein } \\
\text { Schmerz }\end{array}$
\end{tabular}

3a. Bitte kreuzen Sie hier den Schmerz an, den Sie innerhalb der letzten 24 Stunden hatten, wenn Sie sich ruhig verhalten haben.

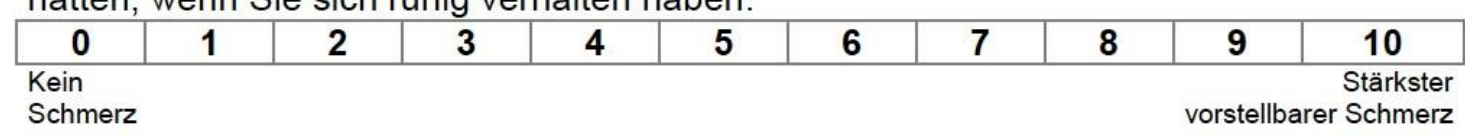

3b Wie stark waren Ihre Schmerzen innerhalb der letzten 24 Stunden, wenn Sie sich belastet haben, zum Beispiel bei Mobilisierung, Bewegen, Waschen, Husten, Durchatmen?

\begin{tabular}{|c|c|c|c|c|c|c|c|c|c|c|}
\hline $\mathbf{0}$ & $\mathbf{1}$ & $\mathbf{2}$ & $\mathbf{3}$ & $\mathbf{4}$ & $\mathbf{5}$ & $\mathbf{6}$ & $\mathbf{7}$ & $\mathbf{8}$ & $\mathbf{9}$ & $\mathbf{1 0}$ \\
\hline $\begin{array}{l}\text { Kein } \\
\text { Schmerz }\end{array}$
\end{tabular}

3c. Bitte kreuzen Sie hier den stärksten Schmerz an, den Sie innerhalb der letzten $24 \mathrm{~h}$ hatten.

\begin{tabular}{|l|l|l|l|l|l|l|l|l|l|r|}
\hline $\mathbf{0}$ & $\mathbf{1}$ & $\mathbf{2}$ & $\mathbf{3}$ & $\mathbf{4}$ & $\mathbf{5}$ & $\mathbf{6}$ & $\mathbf{7}$ & $\mathbf{8}$ & $\mathbf{9}$ & $\mathbf{1 0}$ \\
\hline $\begin{array}{l}\text { Kein } \\
\text { Schmerz }\end{array}$
\end{tabular}

Abbildung 3: Angaben zu empfundenen Schmerzintensitäten

\subsubsection{Funktionelle Beeinträchtigungen}

Da diese empfundenen Schmerzen zum Teil auch andere wesentliche und für den Heilungsverlauf relevante Beeinträchtigungen mit sich bringen können, wurden auch diese in Frage 4 in dichotomer Form mit „ja oder nein“ Antworten erfragt (Abbildung 4). Der Fokus lag dabei auf der Einschätzung der aktuellen Schmerzen. Der Patient konnte angeben, ob er sich in den letzten 24 Stunden durch seine Schmerzen in der Bewegung, beim Husten oder tiefen Luftholen, beim Schlafen oder in seiner Stimmung beeinträchtigt gefühlt hat.

Die Inhalte der Fragen 4 und 5 entsprechen in der konkreten Formulierung den Fragen im QUIPS-Fragebogen, wenn auch dort wieder ein engerer Bezug zur Operation besteht. 
Die nächsten vier Fragen beziehen sich darauf, ob bestimmte Tätigkeiten oder Ihre Stimmung innerhalb der letzten 24 Stunden durch den Schmerz beeinträchtigt waren. Mit ",beeinträchtigt" ist gemeint: die Tätigkeit ist unmöglich oder nur unter großer Mühe möglich. Waren Sind Sie durch die Schmerzen beeinträchtigt:

\section{4a. bei Bewegung?}

口 Ja a Nein

4b. beim Husten oder tiefen Luftholen?
口 Ja
Nein

4c. beim Schlafen?

$$
\text { a Ja Nein }
$$

4d. in Ihrer Stimmung?

口 Ja a Nein

Abbildung 4: Funktionelle Beeinträchtigungen

\subsubsection{Therapiebedingte Nebenwirkungen oder weitere Symptome}

Zur Bewertung des aktuellen Allgemeinzustand des Patienten, wurde in Frage 5 erfasst, ob der Patient innerhalb der letzten 24 Stunden unter starker Müdigkeit, Übelkeit, Schwindel oder Verstopfungen gelitten hatte (Abbildung 5). Die möglicherweise empfundenen Beeinträchtigungen und Nebenwirkungen dienen einer ganzheitlichen Einschätzung der Gesamtqualität der Schmerztherapie und der allgemeinen Symptomlast der befragten Patienten.

Die nächsten 4 Fragen beziehen sich auf Ihren Allgemeinzustand in den letzten 24 Stunden:

5a. Haben Sie sich sehr müde gefühlt?
口 Ja
a Nein

5b. Haben Sie unter Übelkeit gelitten?
口 Ja
Nein

5c. Haben Sie unter Schwindel gelitten?

a Ja a Nein

5d. Leiden Sie derzeit unter Verstopfung?
a Ja
Nein

Abbildung 5: Therapiebedingte Nebenwirkungen oder weitere Symptome 


\subsubsection{Patienteninformation, Patientenbewertung und Zufriedenheit}

Die abschließenden Fragen 6 bis 8 bezogen sich auf die Patienteninformation zum Thema Schmerztherapie durch das Stationsteam und auf die subjektive Bewertung der Qualität einer möglicherweise erhaltenen Schmerztherapie (Abbildung 6). Gleichzeitig hatte der Patient hier die Gelegenheit den Wunsch oder Bedarf an mehr Schmerzmitteln anzugeben. Diese Frage hat sich in QUIPS als relevanter Hinweis auf eine ausreichende oder nicht ausreichende Schmerztherapie erwiesen, unabhängig von definierten Interventionsgrenzen der subjektiv berichteten Schmerzstärke.

Nachdem der Fragebogen vollständig ausgefüllt wurde, konnte der Befragende abschließend noch vermerken, ob der Patient den Fragebogen selbstständig ausgefüllt hat oder ob ein Interview durchgeführt wurde.

6. Wurden Sie über die verschiedenen Möglichkeiten Ihrer Schmerztherapie informiert?
口 Ja
ㅁ Nein

7. Hätten Sie gewünscht, mehr Mittel gegen Schmerzen zu bekommen? 口 Ja a Nein

8. Wenn Sie Schmerzen während Ihres Aufenthaltes im Krankenhaus hatten bzw. Schmerztherapie erhalten haben (z.B. Medikamente, Schmerzsalben, Schmerzkatheter, Krankengymnastik, Wärme, Kälte, Gespräch usw.) kreuzen Sie bitte an, wie zufrieden Sie mit dem Ergebnis der Behandlung Ihrer Schmerzen sind.

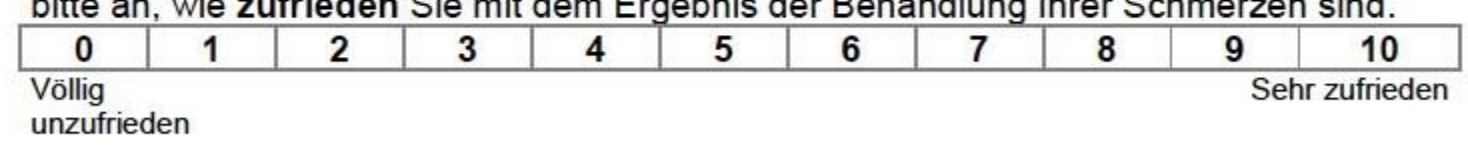

$\square$ Ich hatte keine Schmerzursache/nie Schmerzen und habe auch keine Schmerzbehandlung erhalten.

Abbildung 6: Fragen zur Patienteninformation und Patientenzufriedenheit

\subsection{Prozessparameter}

Die Prozessparameter wurden mithilfe der Patientenkurve und Patientenakte erhoben. Hier wurden zuerst grundsätzlich allgemeine demographische Angaben erfasst, wie z. B. die Station, die jeweilige Fachdisziplin, das Alter und Geschlecht und der Aufnahmetag (Abbildung 7). Das Alter wurde in diesem Zusammenhang nur als Dekade und nicht als konkrete Jahreszahl erfasst. 
D2 Geschlecht: $\square$ männlich $\square$ weiblich

\begin{tabular}{|c|c|c|}
\hline $\begin{array}{l}3 \text { Alter: } \\
61-70\end{array}$ & $\begin{array}{l}\text { 18-20 } \\
\text { 71-80 }\end{array}$ & $\begin{array}{l}\square \text { 21-30 } \\
\text { 口 81-90 }\end{array}$ \\
\hline
\end{tabular}

D4 Kalenderwoche Aufnahme:

_

D6 Fachabteilung:

Innere Medizin ohne Spezialisierung

Kardiologie

Gastroenterologie

- Nephrologie

口 Pulmologie

Rheumatologie

口 Häm./ Onkologie

Endokrinologie

- Neurologie

Psychiatrie

P Psychosomatik

Dermatologie

- Palliativmedizin

sonstige:

D7 Befragungstag nach stationärer Aufnahme:
$\square 1 \square 2$
물
口 4
$\square 5$
$\square 6$
口 7 nicht erhebbar

Abbildung 7: Erhobene Prozessparameter

Neben diesen demographischen Daten wurden die Haupt- und Nebendiagnosen sowie eine eventuelle Schmerzmedikation erhoben. Hier wurde auch der Grund vermerkt, warum ein Patient möglicherweise nicht befragt werden konnte (Abbildung 8).

Zur Erfassung der Diagnosen und Vormedikationen wurden vor allem die aktuelle Stationsdokumentation, sowie neue und ältere Arztbriefe genutzt.

Auf der ersten Seite der Prozessparameter bestand für den Befragenden auch die Möglichkeit, zusätzlich interessante individuelle Parameter oder Fragestellungen über den Patienten in acht Freifelder eintragen zu können. Dies konnten z. B. spezielle Infektionskrankheiten wie MRSA, 3-/4-MRGN oder VRE sein, die zu Isolationsgründen führten und dienten auch zur Überprüfung der Dokumentation in Bezug auf potentiellen Änderungsund Anpassungsbedarf. 


\section{D8 Einschluss des Patienten nicht möglich aus folgendem Grund:}

Patient befindet sich auf einer anderen Station

Patient lehnt die Befragung ab

$\square$ Patient ist verwirrt/hat Verständnisschwierigkeiten

$\square$ Patient ist sediert / schläft

falls ja, bitte auswählen:

$\square$ leicht schläfrig

häufig müde, leicht erweckbar

tief schlafend, schwer erweckbar (Bitte unbedingt Prozessparameter erheben!)

- Sonstige Gründe
Patient ist nicht anwesend / bereits entlassen

$\square$ Patient hat bereits teilgenommen

Patient spricht kein Deutsch

Abbildung 8: Liste möglicher Ausschlusskriterien

\subsubsection{Haupt- und Nebendiagnose}

Für die Erfassung der Haupt- und Nebendiagnose wurde eine Wortdiagnoseliste entwickelt, in der den einzelnen Organsystemen bereits verschiedene „häufige " und „typische“ Diagnosen zugeordnet waren (Abbildung 9). Die verschiedenen Organsysteme verteilten sich auf Herz, Kreislauf, Magen/Darm, Leber/Pankreas/Milz, Stoffwechsel/Hormone, Niere, Lunge, Nervensystem, Haut, Bewegungsapparat, Infektionen, Blut und Maligne Neoplasien.

Hier wurde per Konvention festgelegt, dass die aktuell vorliegende Hauptdiagnose, die zur Aufnahme geführt hat, umkreist und mögliche Nebendiagnosen angekreuzt werden. Sollte eine Hauptdiagnose zum gegenwärtigen Zeitpunkt noch nicht klar zu differenzieren sein, wurde vermerkt, dass diese momentan „nicht erhebbar“ war und es wurde die aktuell vorliegende Symptomatik (z. B. Gangunsicherheit), mit welcher sich der Patient in der Klinik vorgestellt hat, dokumentiert. Für den Fall, dass ein Patient unter einer klar definierten Erkrankung litt, die aber nicht in der Wortdiagnoseliste geführt wurde, wurde diese unter der Rubrik „sonstige Hauptdiagnose“ ergänzt. Dies soll in Zukunft eine weitere Optimierung des Dokuments ermöglichen.

Viele Diagnosen eines konservativen (nicht operativen) Patienten sind nicht klar als Haupt- und Nebendiagnose voneinander abzugrenzen, wodurch hier die Erfassung als Benchmarking-Faktor komplizierter erscheint. Zudem ändert sich häufig bei vielen konservativ behandelten Patienten im stationären Verlauf die Hauptdiagnose, wodurch es zu Abweichungen von Aufnahme- und Entlassungsdiagnose kommt. Die Diagnose-Verschlüsselung für die DRG erfolgt mittels ICD-10, die auch meist erst zur oder nach Entlassung des Patienten endgültig erfolgt. Zum Vergleich werden im QUIPS-Fragebogen die mithilfe des Operationen- und Prozedurenschlüssel (OPS) kodierten Interventionen in der 
Klinik erfasst. Diese OPS definieren klar, warum und auf welche Art und Weise der Patient operiert wurde und sind so für ein Benchmarking in Bezug auf postoperative Schmerzen sinnvoll und geeignet. Die OPS stehen bei konservativ behandelten Patienten dagegen meist nicht im Zusammenhang mit der erlebten Schmerzsymptomatik.

Zudem sind auf den Stationen auch nicht immer die korrekten individuellen ICD-10-Kodierungen einsehbar, wodurch eine genaue Diagnosezuordnung nach dieser Methode nicht möglich ist. Bei QUIKS wurde deshalb nach der ersten Testphase entschieden mit Wortdiagnosen statt mit dem ICD-10 zu arbeiten. Für die Wortdiagnosen ergibt sich bei der Befragung ein größerer Ermessensspielraum durch den Befragenden, um einen Patienten am Befragungstag am zutreffendsten einordnen zu können. 


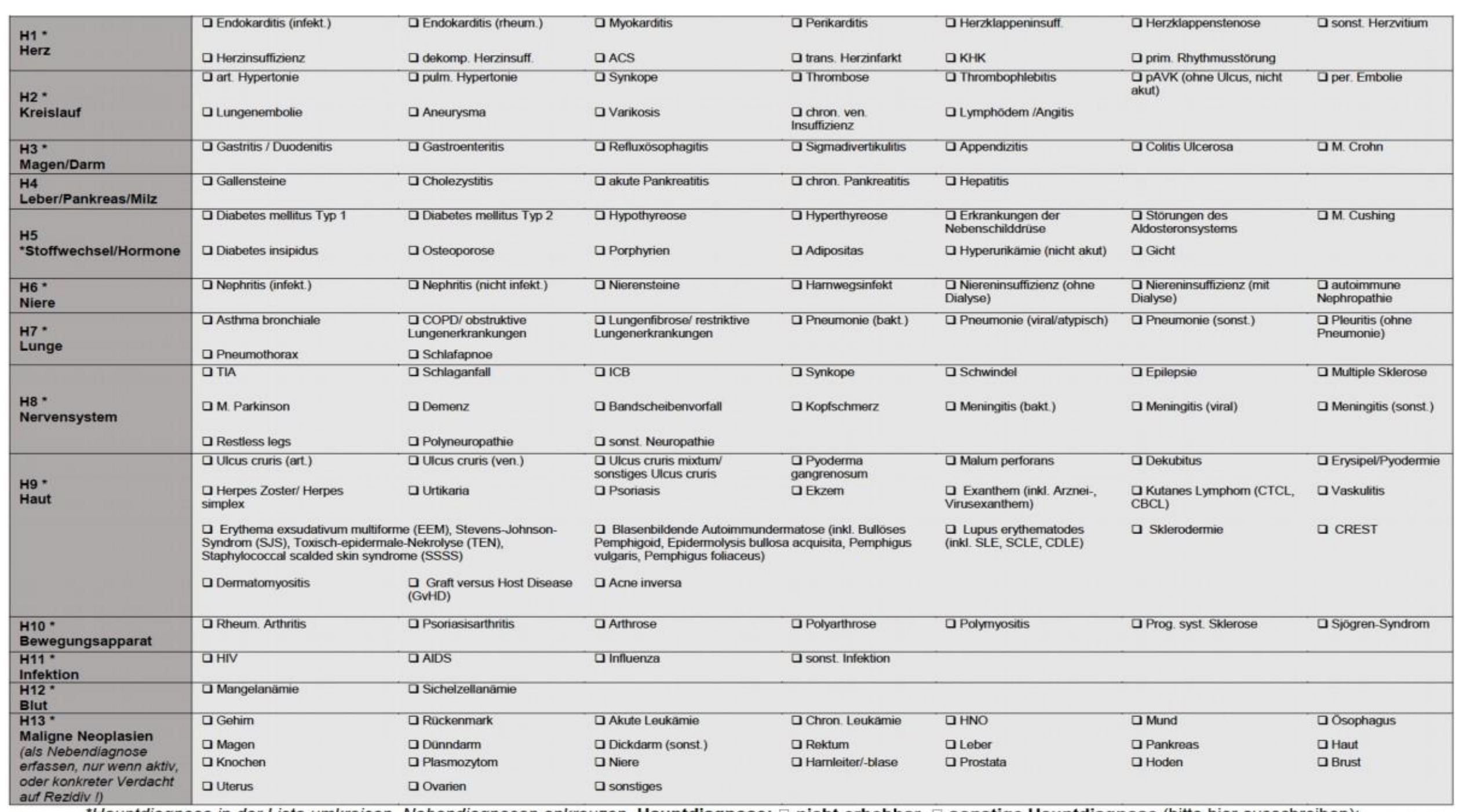

*Hauptdiagnose in der Liste umkreisen, Nebendiagnosen ankreuzen Hauptdiagnose: $\square$ nicht erhebbar $\square$ sonstige Hauptdiagnose (bitte hier ausschreiben):

Abbildung 9: Übersicht der Wortdiagnosen innerhalb der spezifischen Organsystem 


\subsubsection{Schmerzmedikation}

In Bezug auf die Schmerzmedikation (Abbildung 10) wurden sowohl eine schmerzrelevante Vormedikation als auch die stationäre Medikation erfasst. Bei der stationären Schmerzmedikation konnte noch zusätzlich der Vermerk angekreuzt werden, ob diese der Vormedikation entsprach. Grundsätzlich wurde auch erhoben, ob bereits Opioide aufgrund chronischer Schmerzen eingenommen wurden. Des Weiteren konnten auch spezielle schmerztherapeutische Verfahren (z. B. PCA-Pumpen), wie sie beispielsweise in der postoperativen Schmerztherapie häufig eingesetzt werden, aufgenommen werden. Die Wirkstoffgruppen der Medikamente wurden in „Nicht-Opioid-Analgetika“ (z. B. Metamizol, Ibuprofen, Diclofenac oder Paracetamol), in „Opioide“ (z. B. Tilidin oder Fentanyl) und in „Ko-Analgetika“ (z. B. Pregabalin oder Gabapentin) unterteilt. Alternativ konnten auch nicht aufgelistete Medikamente unter der Rubrik „andere“ aufgenommen werden. Als Dosierung wurde die gesamte Tagesdosierung vom Vortag vor der Befragung eingetragen. Zur weiteren Differenzierung wurden neben den jeweiligen Dosierungen auch die unterschiedlichen Applikationsmöglichkeiten (z. B. per os oder intravenös) unterschieden.

Einen Sonderfall stellte die Gabe des Nicht-Opioids ASS in einer Dosierung kleiner $500 \mathrm{mg}$ dar. ASS besitzt in solch einer niedrigen Dosierung keine analgetische Wirkung, sondern wird in der Regel zur Thrombozytenaggregationshemmung (z. B. 100 mg p. o.) eingesetzt (Höllt und Allgaier 2013). Daher wurde ASS in dieser Dosierung zwar tabellarisch erfasst, aber nicht als verabreichtes, analgetisch wirksames Nicht-Opioid gewertet. 
Welche systemische Schmerztherapie wurde vor stationärer Aufnahme durchgeführt (feste Vormedikation?

Hat der Patient vor seinem Krankenhausaufenthalt Opioide gegen chronische Schmerzen genommen? $\square$ ja $\square$ nein $\square$ nicht erhebbar

\begin{tabular}{|c|c|c|c|c|c|c|c|c|}
\hline A1 Nicht-Opioid & \multicolumn{4}{|c|}{$\square$ keins gegeben } & \multicolumn{4}{|c|}{ nicht erhebbar } \\
\hline Applikation & \multicolumn{2}{|c|}{ p.o. } & \multicolumn{2}{|c|}{ i.v. } & \multicolumn{2}{|c|}{ i.m. } & \multicolumn{2}{|c|}{ supp. } \\
\hline Celecoxib & 口 & $\mathrm{mg}$ & & & & & & \\
\hline Etoricoxib & a & $\mathrm{mg}$ & & & & & & \\
\hline Parecoxib & & & $\square$ & $\mathrm{mg}$ & & & & \\
\hline ASS & Q & $\mathrm{mg}$ & D & $\mathrm{mg}$ & & & & \\
\hline Ibuprofen & 口 & $\mathrm{mg}$ & & & & & ב & $\mathrm{mg}$ \\
\hline Diclofenac & 口 & $\mathrm{mg}$ & ב & $\mathrm{mg}$ & ב & $\mathrm{mg}$ & $\square$ & $\mathrm{mg}$ \\
\hline Indometacin & D & $\mathrm{mg}$ & & & & & 口 & $\mathrm{mg}$ \\
\hline Meloxicam & 口 & $\mathrm{mg}$ & & & & & & \\
\hline Naproxen & 口 & $\mathrm{mg}$ & & & & & & \\
\hline Metamizol & 口 & $\mathrm{mg}$ & 口 & $\mathrm{mg}$ & 口 & $\mathrm{mg}$ & 口 & $\mathrm{mg}$ \\
\hline Paracetamol & 口 & $\mathrm{mg}$ & 口 & $\mathrm{mg}$ & ב & $\mathrm{mg}$ & ב & $\mathrm{mg}$ \\
\hline
\end{tabular}

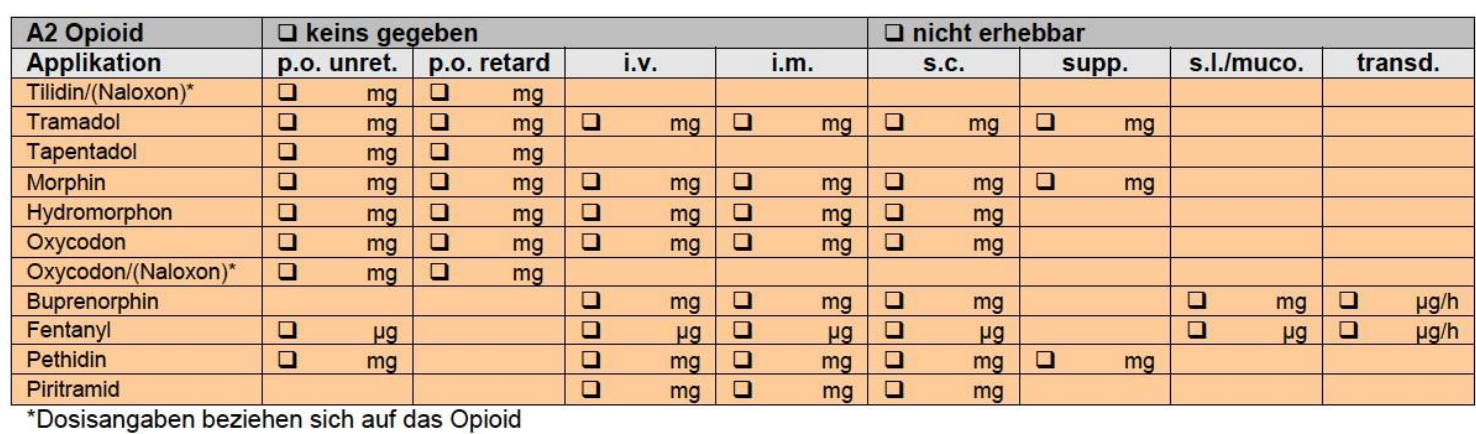

\begin{tabular}{|c|c|c|c|c|c|c|c|c|c|c|c|c|}
\hline \multirow{3}{*}{$\begin{array}{l}\text { A3 Ko-Analgetika } \\
\text { Applikation } \\
\text { Pregabalin }\end{array}$} & \multicolumn{4}{|c|}{$\square$ keins gegeben } & \multicolumn{8}{|c|}{$\square$ nicht erhebbar } \\
\hline & \multicolumn{2}{|c|}{ p.o. } & \multicolumn{2}{|c|}{ retard } & \multicolumn{2}{|c|}{ i.v. } & \multicolumn{2}{|c|}{ i.m. } & \multicolumn{2}{|c|}{ s.c. } & \multicolumn{2}{|c|}{ supp } \\
\hline & $\square$ & $\mathrm{mg}$ & & & & & & & & & & \\
\hline Gabapentin & $\square$ & $\mathrm{mg}$ & & & & & & & & & & \\
\hline Carbamazepin & $\square$ & $\mathrm{mg}$ & $\square$ & $\mathrm{mg}$ & & & & & & & & \\
\hline Amitryptilin & $\square$ & $\mathrm{mg}$ & $\square$ & $\mathrm{mg}$ & $\square$ & $\mathrm{mg}$ & $\square$ & $\mathrm{mg}$ & $\square$ & $\mathrm{mg}$ & & \\
\hline Trimipramin & $\square$ & $\mathrm{mg}$ & & & & & & & & & & \\
\hline Duloxetin & $\square$ & $\mathrm{mg}$ & & & & & & & & & & \\
\hline Citalopram & $\square$ & $\mathrm{mg}$ & & & $\square$ & $\mathrm{mg}$ & & & & & & \\
\hline Sertralin & $\square$ & $\mathrm{mg}$ & & & & & & & & & & \\
\hline Doxepin & 口 & $\mathrm{mg}$ & & & $\square$ & $\mathrm{mg}$ & $\square$ & $\mathrm{mg}$ & & & & \\
\hline Lorazepam & $\square$ & $\mathrm{mg}$ & & & 口 & $\mathrm{mg}$ & & & & & & \\
\hline Diazepam & 口 & $\mathrm{mg}$ & & & 口 & $\mathrm{mg}$ & $\square$ & $\mathrm{mg}$ & & & $\square$ & $\mathrm{mg}$ \\
\hline Flupirtin & $\square$ & $\mathrm{mg}$ & $\square$ & $\mathrm{mg}$ & $\square$ & $\mathrm{mg}$ & $\square$ & $\mathrm{mg}$ & & & $\square$ & $\mathrm{mg}$ \\
\hline
\end{tabular}

andere:

Abbildung 10: Aktuelle Medikamentenauswahl 
Abschließend wurde anhand der Patientenkurve auch erfasst, ob für den Patienten generell eine Schmerztherapieanordnung bestand und diese eine Basis- und/oder eine Bedarfsmedikation beinhaltete (Abbildung 11). Durch Sichtung der Patientenkurve wurde ebenfalls erfasst, ob in den vergangenen 24 Stunden eine Schmerzdokumentation durch das Stationsteam durchgeführt wurde (z. B. mittels NRS).

Ist eine Individuelle Therapieanordnung für die Schmerztherapie auf der Station vorhanden?

\begin{tabular}{|l|c|c|}
\hline S4 Therapieanordnung & $\square \mathrm{Ja}$ & $\square \mathrm{Nein}$ \\
\hline Basisanalgesie angeordnet & $\square \mathrm{Ja}$ & $\square \mathrm{Nein}$ \\
\hline Bedarfsmedikation angeordnet & $\square \mathrm{Ja}$ & $\square \mathrm{Nein}$ \\
\hline
\end{tabular}

Ist eine Schmerzdokumentation (z.B. Kurve) auf der Station erfolgt? \begin{tabular}{|l|l|l|}
\hline S5 Schmerzdokumentation & $\square \mathrm{Ja}$ & $\square$ Nein \\
\hline
\end{tabular}

Abbildung 11: Individuelle Therapieanordnung (Basis- und/oder Bedarfsmedikation) und Schmerzdokumentation

Sollte der Patient bei der persönlichen Rückgabe einzelne Fragen nicht beantwortet haben, wurden die dafür vorliegenden Gründe erfragt. Wenn es bei der jeweiligen Frage Verständnisschwierigkeiten gab, konnten so noch einmal Hilfestellungen gegeben werden. Die subjektive Patienteneinschätzung wurde hierbei selbstverständlich nicht beeinflusst, um keine Verfälschungen der Ergebnisse zu verursachen. Für den Fall, dass ein Patient bei der Rückgabe nicht mehr persönlich anzutreffen war, wurden die unvollständig ausgefüllten Fragebögen eingesammelt und die betroffenen Felder bei der stets unmittelbar folgenden Digitalisierung als „fehlend“ eingetragen.

Von den Patienten, die eine Befragung abgelehnt haben, wurden zur Vollständigkeit der Studie nur die demographischen Daten und das Ausschlusskriterium (z. B. „Patient ist nicht anwesend" oder "lehnt die Befragung ab") aufgenommen (Abbildung 7 und Abbildung 8).

\subsection{Quantitative und qualitative Auswertung}

Die Auswertung der erhobenen Daten erfolgte mithilfe von Microsoft Excel 2016 und SPSS Statistics Version 24 von der Firma IBM.

Zu Beginn wurde eine rein deskriptive Auswertung der erhobenen quantitativen Daten vorgenommen. Die jeweiligen NRS-Werte wurden mit dem Median und in Klammern mit dem 25\% bzw. 75\% Quartil (Q1 bzw. Q3) angegeben. In der anschließenden qualitativen 
Bewertung der Konstruktvalidität wurde eine explorative Faktorenanalyse und eine Hauptkomponentenanalyse mit Promax-Rotation durchgeführt. Dieses Vorgehen wurde mit dem Kaiser-Meyer-Olkin-Kriterium (KMO) und dem Bartlett-Test gerechtfertigt. Diese Analyse folgte dem Vorgehen bei der Validierung von QUIPS und PAIN OUT (Rothaug et al. 2013).

Die Reliabilität wurde mithilfe der internen Konsistenz nach Cronbachs a getestet. Zur Überprüfung der klinischen oder externen Validität wurden die Schmerzintensitäten und funktionellen Beeinträchtigungen von bekanntermaßen klinisch sehr unterschiedlichen Hauptdiagnosen (in Bezug auf begleitende Schmerzsymptomatik) mit dem Mann-Whitney-U-Test verglichen. Um eine etwa gleich große Anzahl von Patienten vergleichen zu können wurden die Hauptdiagnosen Schlaganfall und TIA zu einer Gruppe zusammengefasst und mit der Gruppe von allen zusammengefassten malignen Erkrankungen verglichen. Für das Signifikanzniveau wurde den Messungen ein p-Wert von 0,05 zugrunde gelegt.

Eine Auswertung der Ergebnisse der Zufriedenheit des gesamten Patientenkollektiv wurde als nicht sinnvoll erachtet und nicht durchgeführt. Um ein möglichst aussagekräftiges Ergebnis über die subjektive Qualität der Schmerztherapie erhalten zu können, wurde daher überlegt welche Patientengruppe mit einer konkreten Schmerzangabe im Fragebogen am ehesten von dieser Situation betroffen sein könnte. Es wurden zum einen Patienten mit vorbestehenden chronischen Schmerzen und zum anderen mit Schmerzen bei der Aufnahme oder während des stationären Aufenthaltes ausgewählt. Für QUIKS ergaben sich daher mehrere verschiedene Möglichkeiten zur Auswertung.

Es muss vorab festgehalten werden, dass es bei dieser differenzierten Auswertung auch Überschneidungen geben konnte.

Um mit QUIKS eine gute methodische Qualität abbilden zu können, wurde das Fragebogen- und Studiendesign mit der COSMIN-Checkliste („COnsensus-based Standards for the selection of health Measurement INstruments"; Stand 2012) überprüft. 


\subsection{Ethikantrag}

Die Ethik-Kommission der Universität Göttingen hat das vorliegende Projekt mit der Antragsnummer 34/2/16 am 18.02.2016 beraten und demgegenüber keine ethischen oder rechtlichen Bedenken geäußert.

Gleichzeitig bestehen für die Ethik-Kommission keine Bedenken gegen die Mitarbeit des Doktoranden bei einem medizinischen Forschungsprojekt und dem vorliegenden Dissertationsvorhaben (Antragsnummer DOK_59_2016).

Bereits für die erste Projektphase vor dieser Promotionsarbeit, zur initialen Testung des Fragebogens auf Verständlichkeit und Umsetzbarkeit, wurde ein positives Ethik-Votum an der Universität Göttingen eingeholt (Antragsnummer 10/2/14 vom 05.02.2014). 


\section{Ergebnisse}

Die Ergebnisse gliedern sich in einen deskriptiven Teil über die Test- und Projektphasen, sowie einen an die Hauptprojektphase anschließenden ersten qualitativen Bewertungsversuch des neuen Erhebungsinstruments. Vor der eigentlichen Projektphase wurden drei Testphasen durchgeführt, um Verständnisprobleme und Schwierigkeiten innerhalb des Ergebnisfragebogens und den Prozessparametern zu detektieren. Dazu gehörten auch die Erprobung von Durchführungs- und Dokumentationsabläufen auf den jeweiligen Stationen und in der Excel-Benchmarking-Datei durch den Befragenden selbst. Innerhalb dieser Testphasen wurden auch die späteren Einschlusskriterien (z. B. Alter oder Aufnahmetag; Tabelle 2) für die Patienten festgelegt und erprobt. Gleichzeitig konnte auch so ein erster Eindruck von der Umsetzbarkeit und Anwendbarkeit des Projekts gewonnen werden.

\subsection{Testphasen}

\subsubsection{Zweite Testphase}

In der ersten Voruntersuchung zeigte sich, dass zum gegenwärtigen Zeitpunkt kein einheitlicher Befragungstag vorgelegen hat. Um jedoch ein validierbares Ergebnis zu erhalten, wurde ab dieser zweiten Phase der einheitlich dritte Krankenhaustag als Befragungstag festgelegt. Insgesamt konnten in dieser Phase 42 Patienten befragt werden, von denen 4 eine Befragung abgelehnt haben. Da der Zugriff auf die Bettenbelegungspläne durch den Befragenden zu Beginn noch eingeschränkt war, konnten hier nur 59\% der Patienten korrekt am dritten Aufnahmetag befragt werden. Die Ergebnisse der übrigen Patienten wurden jedoch nicht verworfen, sondern wurden unter Berücksichtigung dieser Einschränkung mit einbezogen.

Die in dieser Testphase teilnehmenden Kliniken waren die Klinische Neurophysiologie ( $n=23)$, die Dermatologie $(n=11)$ und die Gastroenterologie $(n=8)$ an der Universitätsmedizin Göttingen.

In der demographischen Verteilung teilten sich die Patienten wie bereits in der Voruntersuchung und auch in der abschließenden Hauptprojektphase nahezu gleichmäßig in 52\% männlich und 48\% weiblich auf. Der größte Teil der befragten Patienten (26\%) befand 
sich ebenfalls in der Altersdekade von 51 bis 60 Jahre. Insgesamt wurde bei 24\% der Patienten die Befragung in Form eines Interviews durchgeführt.

Eine einwandfreie Dokumentation der Wortdiagnosen in die Benchmarking-Datei stellte zu Beginn eine Schwierigkeit dar, die bis zur Projektphase verfeinert und optimiert wurde. Die allgemeine praktische Anwendbarkeit der Wortdiagnoseliste vor Ort wurde jedoch sehr schnell deutlich. Die aktuell gültige Hauptdiagnose konnte gut in umkreisender Form und laufenden Nebendiagnosen durch ankreuzen dokumentiert werden. Dieses Vorgehen wurde in allen Phasen dieses Projekts beibehalten und wird auch voraussichtlich so abschließend in der QUIKS-Verfahrensanweisung implementiert werden.

Die am häufigsten anzutreffenden Hauptdiagnosen waren, unter Berücksichtigung der teilnehmenden Fachdisziplinen, die Epilepsie $(n=4)$, M. Parkinson $(n=3)$ und die Multiple Sklerose $(n=2)$. In zehn Fällen wurde eine sonstige Hauptdiagnose angegeben.

Im Rahmen der Dokumentation der Schmerzmedikation gab es keine Schwierigkeiten und die Vorlage konnte problemlos umgesetzt werden. Auf allen Stationen konnten unmissverständlich die jeweilige Medikation, Dosierung und Applikationsform erfasst werden. Metamizol, Diclofenac und Ibuprofen waren die gebräuchlichsten Nicht-Opioid-Analgetika der Vormedikation, im Rahmen der innerklinischen Schmerztherapie wurde noch zusätzlich Paracetamol eingesetzt.

In der Vormedikation wurden bereits in sechs Fällen Opioide gegen Schmerzen eingenommen. Im Vergleich hierzu wurde drei Patienten mehr Opioide auf den Stationen verordnet. Als Wirkstoffe wurden vor allem Oxycodon in Kombination mit Naloxon und retardiertes Tilidin, Tramadol, Morphin, Buprenorphin, Pethidin und Fentanyl eingesetzt. Koanalgetisch fanden sowohl in der Vormedikation als auch in der Klinikmedikation vor allem Pregabalin, Gabapentin und Citalopram Anwendung.

Die Rubrik der schmerzrelevanten „anderen Medikamente“ wurde im Verlauf der Testphase konkret festgelegt. Im vorliegenden Fall sollten die Wirkstoffgruppen Valproinsäure, Topiramat, Flunarizin, Mirtazapin, Venlafaxin, Tizanidin, Baclofen, Tetrahydrocannabinol, Triptane und Oxcarbazepin miterfasst werden. Am häufigsten wurde hier jeweils Mirtazapin eingesetzt. 


\subsubsection{Dritte Testphase}

In dieser Zeit stand unabhängig von den Prozessparametern die Testung der neu eingefügten Tabelle zu verschiedenen Schmerzentitäten an 30 Patienten im Fokus, von der jedoch insgesamt 11 Patienten keinen Gebrauch machten. In der Auswertung der anderen 19 Patienten wurde die Problematik deutlich, dass sich die Patienten auf einen einzigen Schmerz festlegen sollten, den sie als „schlimmsten Schmerz" bezeichnen würden. Vielfach kam es hier zu einer nicht vollständig korrekten Beantwortung der Frage $(n=15)$, da die Patienten mehrere oder gar keine Angaben machten.

Als generelle Probleme zur Durchführung wurden dem Befragenden mehrfach Verständnisschwierigkeiten einzelner zu komplexer Fragestellungen, ein insgesamt reduzierter Allgemeinzustand und Sprachbarrieren zurückgemeldet. Ein dermatologischer Patient gab ebenfalls an, Schwierigkeiten mit der "Definition Schmerz" in der Frage selbst zu haben, da er unter starkem Juckreiz leiden würde und sich nicht sicher sei, ob er diese Bewertung dann mit in die Beantwortung einfließen lassen solle oder nicht. Die weiteren Ergebnisse dieser Testphase entsprechen weitgehend denen der übrigen Phasen. Die Tabelle mit der Schmerzeinschätzung wurde trotz der Schwierigkeiten in die Projektphase übernommen.

\subsection{Projektphase: Quantitative Auswertung des Ergebnisfragebogens}

Der Ergebnisfragebogen und die Prozessparameter wurden nach den drei Testphasen für die abschließende Projektphase im Rahmen dieser Promotionsarbeit freigegeben. Hier zeigte sich auch an der nun größeren Stichprobe, dass die Patienten mit einem Teil der Fragen völlig problemlos umgehen konnten, bei anderen Fragen entstanden häufige Nachfragen oder inkonsistente Antworten in der Folge. Besonders die komplexeren Fragestellungen, die korrekte Anwendung der neuen Tabelle für die empfundenen Schmerzentitäten und Angaben zur Patientenzufriedenheit stellten eine wiederkehrende Fehlerquelle dar. Diese Problemfälle werden im Verlauf detailliert aufgeführt.

Insgesamt war es in der Hauptprojektphase möglich, dass 99\% der Patienten auch letztlich am 3. Aufnahmetag befragt wurden. Aufgrund von z. B. schlechter Dokumentation auf Station bzw. durch Missverständnisse wurden weniger als 1\% der Patienten an einem falschen Tag befragt. 
Die Fehlerrate konnte durch gut geführte Belegungspläne und mit Hilfe von ixserv.4 sehr gering gehalten und im Vergleich zur Testphase deutlich verbessert werden.

In der demographischen Pyramide (Abbildung 12) verteilten sich die Geschlechter mit 51\% Männern und 49\% Frauen nahezu gleich. Das Alter der Patienten zeigte, wie in den Testphasen zuvor, eine linksschiefe Verteilung $(-0,627)$ vor allem zwischen der 50 . und 70. Lebensdekade, mit dem Modalwert von 27\% beider Geschlechter im Alter von 71 bis 80 Jahren.

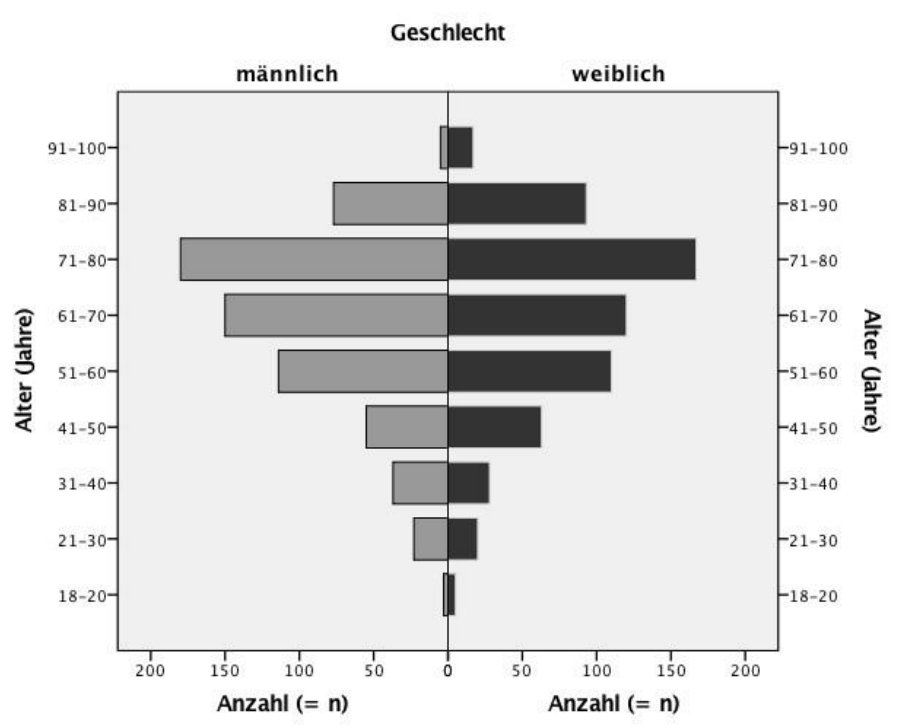

Abbildung 12: Demographische Pyramide

Die im Zeitraum von April bis Oktober 2016 befragten 1317 Patienten verteilten sich auf die konservativen Fächergruppen (Tabelle 3 ) der Inneren Medizin ( $=537)$, Neurologie ( $n=564)$ und Dermatologie $(n=216)$.

Zur Inneren Medizin zählten hier die 12\% der Patienten in der Kardiologie, 3\% in der Pulmologie, 19\% in der Gastroenterologie, 1\% in der Hämatologie/Onkologie, 4\% in der Nephrologie und 3\% in der Rheumatologie. Die 564 neurologischen Patienten ergaben 34\% in der Neurologie und 9\% in der Klinischen Neurophysiologie.

Von den gesamten 1317 Patienten haben 74\% einer Befragung zugestimmt, die restlichen $26 \%(n=344)$ konnten aus unterschiedlichen Gründen (Tabelle 2$)$ nicht eingeschlossen werden. Mit 187 Patienten (14\%) war die größte Gruppe der Patienten diese, die am dritten Befragungstag entweder bereits wieder entlassen oder nicht auf der Station anzutreffen waren, trotz in der Regel mehrmaliger Versuche. Gefolgt wurde diese Gruppe von den Patienten ( $n=75 \bumpeq 6 \%$ ), die eine Befragung bewusst abgelehnt haben. Diese 
Patienten gaben dafür unterschiedlichste Beweggründe an, trotz ausführlicher Erklärungen durch den Befragenden. Meistens war es entweder ein mangelndes Interesse, Skepsis gegenüber der Anonymität der Befragung oder der Befragungshintergründe selbst.

Die drittgrößte Gruppe schließt die Patienten ein, die verwirrt waren oder Verständnisschwierigkeiten bei der Befragung hatten $(n=45 \bumpeq 3 \%)$. In diese Gruppe wurden auch alle Patienten aufgenommen, die aus den unterschiedlichsten gesundheitlichen Gründen aktuell nicht in der Lage waren, befragt zu werden.

Im Verlauf wurden die Daten dieser 344 Patienten in den tabellarischen Auswertungen zur besseren Übersicht herausgenommen.

Tabelle 3: Patientenverteilung innerhalb der verschiedenen Fachdisziplinen der Inneren Medizin, Neurologie und Dermatologie

\begin{tabular}{|l|c|c|c|c|}
\hline \multirow{2}{*}{ Fachabteilung } & \multicolumn{2}{|c|}{ alle Patienten } & \multicolumn{2}{|c|}{ kein Einschluss } \\
\cline { 2 - 6 } & $\mathrm{n}$ & Prozent & $\mathrm{n}$ & Prozent \\
\hline Dermatologie & 216 & 16,4 & 47 & 13,7 \\
\hline Gastroenterologie & 250 & 19,0 & 62 & 18,0 \\
\hline Hämatologie/Onkologie & 10 & 0,8 & 4 & 1,2 \\
\hline Kardiologie & 151 & 11,5 & 40 & 11,6 \\
\hline Nephrologie & 55 & 4,2 & 24 & 7,0 \\
\hline Neurologie & 445 & 33,8 & 124 & 36,0 \\
\hline Neurophysiologie & 119 & 9,0 & 39 & 11,3 \\
\hline Pulmologie & 33 & 2,5 & 3 & 0,9 \\
\hline Rheumatologie & 38 & 2,9 & 1 & 0,3 \\
\hline Gesamt (fehlend) & - & - & 344 & 100,0 \\
\hline Gesamt & 1317 & 100,0 & & - \\
\hline
\end{tabular}

\subsubsection{Frage 1: Chronisches Schmerzleiden}

Die abhängige erste Frage, in der die Patienten in Frage 1a angeben sollten, ob vor der Klinikaufnahme bereits ein chronischer Schmerz von einer Länge über 3 Monate bestand, führte ebenfalls an dem großen Patientenkollektiv zu widersprüchlichen Angaben, da die Fragen $1 b$ und $1 c$ nur relevant waren, wenn Frage 1a mit ,ja“ beantwortet wurde. 
Nahezu gleich viele Patienten beantworteten die Frage 1a mit , ja" ( $n=393 \bumpeq 51 \%$ ) oder „nein“ ( $n=383$ ^ 49\%) (Tabelle 4). Auffällig war hierbei jedoch, dass auch insgesamt 197 Patienten (15\%) die gesamte abhängige Frage „falsch“ beantworteten. Hierzu gehörten sowohl Patienten (gültig 196: 109 männlich und 87 weiblich; einmal Geschlecht nicht erfasst), die nicht alle Teilfragen beantworteten, oder die trotz der Angabe „nein“ in Frage 1a ebenfalls entweder Frage $1 \mathrm{~b}$ und/oder Frage 1c beantworteten. Von den Patienten, die Frage 1 korrekt beantworteten und somit einen bestehenden chronischen Schmerz in ihrer Anamnese angaben, waren 174 männlich und 216 weiblich.

Tabelle 4: Gesamtauswertung der Frage 1: Vorbestehendes chronisches Schmerzleiden

\begin{tabular}{|l|c|c|c|}
\hline & Anzahl (n) & Prozent & Gültig (\%) \\
\hline Ja & 393 & 29,8 & 50,6 \\
\hline Nein & 383 & 29,1 & 49,4 \\
\hline Gesamt & 776 & 58,9 & 100,0 \\
\hline Frage falsch beantwortet & 197 & 15,0 & \\
\hline
\end{tabular}

In der Auswertung wurden in Frage 1a alle die Angaben rausgerechnet, die gar nicht angekreuzt wurden und wenn eine oder beide Folgefragen falsch beantwortet wurden. In diesem Fall wurde dann die gesamte Frage als „falsch beantwortet" gewertet. Auffallend war hierbei, dass die meisten Fehler dann entstanden sind, wenn der Patient die Frage 1 a mit „nein" beantwortet hat. Es ergab sich, dass in 109 der 196 gültigen Fälle diese Fragen von Männern als „falsch beantwortet“ gewertet wurden, bei 3 Patienten davon wurde die Frage 1a zuvor mit „ja“ beantwortet. Bei den betreffenden 87 Frauen war dies nur bei 2 Patientinnen der Fall.

Gleichzeitig ließ sich aber festhalten, dass das Alter der Patienten einen auffallenden Einfluss auf die Fehlerquote bei dieser Frage hatte. Die häufigsten Fehler fanden sich in den Lebensdekaden zwischen 50 und 80 Jahren. Die größte Häufigkeit lag hier mit 56 Patienten im Alter von 71 bis 80 Jahre (18 bis 20 Jahre = 3 Patienten, 21 bis 30 Jahre $=7$ Patienten, 31 bis 40 Jahre = 7 Patienten, 41 bis 50 Jahre = 14 Patienten, 51 bis 60 Jahre = 39 Patienten, 61 bis 70 Jahre $=48$ Patienten, 81 bis 90 Jahre $=17$ Patienten und 91-100 Jahre $=2$ Patienten und insgesamt 4 Patienten ohne Altersangaben ). 
In Frage 1b konnten die Patienten die Schmerzintensität der vorbestehenden chronischen Schmerzen mittels NRS angeben. Der Median war hier bei Männern 5 (4 - 7) und bei Frauen $6(5-8)$. Insgesamt lagen in dieser Projektphase knapp 80\% der befragten Patienten mit korrekten Angaben in den vorangegangenen drei Monaten über einem NRS-Wert von 3.

In Frage 1c gaben 170 Patienten (43\%) an, auch wegen dieser chronischen Schmerzen aktuell in die Klinik aufgenommen worden zu sein. Bei 216 (55\%) der befragten Patienten lag ein anderer Aufnahmegrund vor, 7 Patienten (2\%) konnten hierzu keine Aussage geben.

\subsubsection{Frage 2: Schmerzentitäten}

Um die vorliegenden Schmerzen besser lokalisieren zu können, gaben die Patienten mit der eingefügten Tabelle in Frage 2a ihre aktuellen Schmerzentitäten (Abbildung 2 und Tabelle 5) und ihren am schlimmsten empfundenen Schmerz während des Klinikaufenthaltes an. Insgesamt machten 755 (78\%) Patienten von dieser Tabelle Gebrauch, 218 (22\%) Patienten nutzten sie nicht.

Insgesamt machten aber auch 577 Patienten in dieser Frage ungültige Aussagen. Diese wählten entweder gar keinen „schlimmsten Schmerz" aus, oder legten sich nicht auf eine einzelne Aussage dazu fest und kreuzten hier z. B. mehrere Schmerzentitäten an. In der folgenden Auswertung von Frage 2a wurden diese ungültigen Angaben nicht mitberücksichtigt.

Dies bedeutete, dass lediglich 24\% $(n=178)$ der Patienten die Tabelle in der korrekten Form nutzten. Dieser Trend zeichnete sich bereits in der dritten Testphase im kleinen Patientenkollektiv ab und wurde in der großen Projektphase bestätigt.

Von den Patienten, die die Tabelle anwendeten, sind die häufigsten Angaben zu ihren grundsätzlich, unabhängig von ihrer Aufnahmediagnose empfundenen Schmerzen vor allem Rückenschmerzen (48\%), Gelenkschmerzen (39\%), Kopfschmerzen (32\%) und Nackenschmerzen (29\%).

Von diesen Patienten wurde auch der Rückenschmerz (19\%), der Gelenkschmerz (15\%) und der Kopfschmerz (11\%) mit auffallender Mehrheit als „schlimmster Schmerz" empfunden. Mit etwas Abstand folgten dann Angaben zu am schlimmsten empfundenen 
Bauchschmerzen (9\%), Muskelschmerzen (7\%) und mit je 6\% Nervenschmerzen, Schmerzen bei Untersuchungen und andere Schmerzen. Die übrigen Angaben wurden hier in der Auflistung vernachlässigt, da sie nur einen Anteil von weniger als 5\% umfassten.

Unter der Rubrik „andere Schmerzen“ war es den Patienten möglich nicht aufgelistete Schmerzlokalisationen/-entitäten individuell einzutragen. Die Ergebnisse zeigten, dass sich in den meisten Fällen die Eintragungen bereits bestehenden Gruppierungen zuordnen ließen und somit wurde deutlich, dass die Patienten hier sehr große Schwierigkeiten bei der korrekten Anwendung hatten. Als jedoch häufiger genannte fehlende Obergruppe zeigten sich unterschiedliche Angaben zu Schmerzen der Haut.

In den Fragen 2b und 2c konnten die Patienten mittels NRS Aussagen darüber treffen, wie stark die Schmerzen bei der aktuellen Aufnahme in die Klinik waren, bzw. wie stark der stärkste Schmerz bei Behandlungen während dieser Zeit war (Abbildung 13). 
Tabelle 5: Eine Übersicht mit den Angaben zu den empfundenen Schmerzentitäten in der Klinik unabhängig von der Aufnahmediagnose

\begin{tabular}{|l|c|c|c|}
\hline Schmerzentität & $n$ & Antworten (\%) & Fälle (\%) \\
\hline Gesichtsschmerz & 65 & 3,0 & 8,9 \\
\hline Kopfschmerzen & 235 & 10,7 & 32,1 \\
\hline Nackenschmerzen & 211 & 9,6 & 28,8 \\
\hline Rücken-/Kreuzschmerzen & 352 & 16,0 & 48,1 \\
\hline Mund-/Halsschmerzen & 72 & 3,3 & 9,8 \\
\hline Herzschmerzen & 50 & 2,3 & 6,8 \\
\hline Schmerzen im Brustkorb & 105 & 4,8 & 14,3 \\
\hline Bauchschmerzen & 128 & 5,8 & 17,5 \\
\hline Leistenschmerzen & 49 & 2,2 & 6,7 \\
\hline Blasen-/Nierenschmerzen & 59 & 2,7 & 8,1 \\
\hline Gelenk-/Knochenschmerzen & 285 & 12,9 & 38,9 \\
\hline Muskelschmerzen & 177 & 8,0 & 24,2 \\
\hline Nervenschmerzen & 134 & 6,1 & 18,3 \\
\hline Schmerzen bei Durchblutungsstörungen & 59 & 2,7 & 8,1 \\
\hline Wund-/Narbenschmerzen & 90 & 4,1 & 12,3 \\
\hline Schmerzen bei Untersuchung, Eingriff, Punktion, Behandlung & 131 & 5,9 & 17,9 \\
\hline Gesamt & 2202 & 100,0 & 300,8 \\
\hline
\end{tabular}



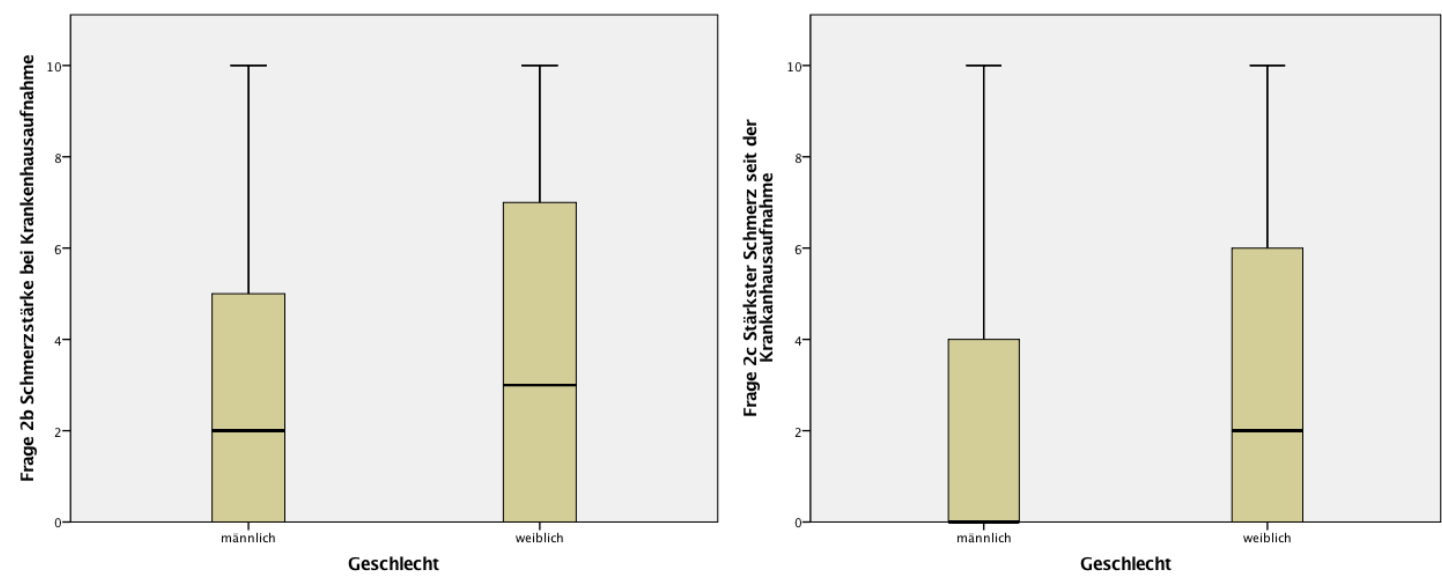

Abbildung 13: Geschlechtsspezifische Angaben zu empfundenen Schmerzintensitäten bei der Klinikaufnahme und bei Prozeduren während des Klinikaufenthalts

Aus beiden Fragen konnte man erkennen, dass ein Großteil der Patienten ( $n=392 \bumpeq 41 \%$ ) bei der Klinikaufnahme akut keine Schmerzen (NRS 0) empfanden. Bei Frage 2b war der Median bei Männern $2(0-5)$ und bei Frauen $3(0-7)$. Insgesamt wurde nach NRS 0 der mittlere Wert bei NRS 5 am häufigsten angegeben (11\%).

Während diagnostischer oder therapeutischer Maßnahmen gaben sogar 47\% der Patienten an, subjektiv keine Schmerzen empfunden zu haben (NRS 0). Hier waren die akuten Schmerzen im Median von männlichen Patienten $0(0-4)$ und bei weiblichen Patienten $2(0-6)$ im von der Leitlinie zur Akutschmerztherapie (DGAI 2007) gewünschten Bereich zur Interventionsgrenze von NRS 3 geblieben. Interventionsgrenzen sollten jedoch möglichst nicht zwischen akuten und chronischen Schmerzen verglichen werden oder pauschal anhand von NRS-Werten festgelegt werden. Stattdessen sollten sie primär am subjektiven Patientenbedarf orientiert werden (Gerbershagen et al. 2011). Deutlich herausstechende Werte, wie bei Frage $2 b$, waren hier nicht zu erkennen. Während dieser Interventionen in den Kliniken musste somit eine von den Patienten subjektiv empfundene gute Information zu möglichen Schmerzen und eine entsprechend suffiziente, bedarfsgerechte Analgesie stattgefunden haben. Auf der anderen Seite wurde mit der Befragung nicht erfasst, ob in der Therapie beim jeweiligen Patienten überhaupt eine schmerzbehaftete Intervention stattfand. 


\subsubsection{Frage 3: Schmerzintensitäten}

In Frage 3 konnten die Patienten ebenfalls mittels NRS Angaben zur Schmerzintensität innerhalb der vergangenen 24 Stunden vor der Befragung in Ruhe, unter Belastungen und als Maximalschmerz machen (Abbildung 14).

52\% der männlichen Patienten gaben im Median an, in Ruhe keine Schmerzen oder einen NRS von $0(0-3)$ zu empfinden. Der Belastungsschmerz bei Männern war im Median mit $1(0-4)$ nur minimal erhöht. 49\% gaben auch hier an, weiterhin keine Schmerzen empfunden zu haben. Der Maximalschmerz innerhalb der vergangenen 24 Stunden wurde von den männlichen Patienten im Median mit 2 (0 - 5) angegeben. Knapp 33\% der Angaben lagen oberhalb von NRS 3.

Frauen gaben in allen drei Teilfragen etwas höhere Schmerzintensitäten an. In Ruhe war der Median $2(0-5)$ und damit über dem der männlichen Patienten. Auch gaben im Vergleich nur 41\% der Patientinnen an, keine Schmerzen (NRS 0) empfunden zu haben. Insgesamt lagen hier knapp 36\% über NRS 3. Unter Belastungen waren subjektiv auch nur noch 38\% der Frauen schmerzfrei (Median 2; 0 - 5). Der maximal empfundene Schmerz war im Median $3(0-6)$. Insgesamt gaben 27\% der Männer und 41\% der Frauen an, unter Belastungen einen Schmerz größer NRS 3 empfunden zu haben.

Die Werte des maximalen Schmerzes wurden in der Auswertung als Kennwert genommen, um zu definieren, wie viele Patienten letztlich überhaupt Schmerzen angaben. Hier zeigte sich, dass erstmal unabhängig von der Intensität, möglichen Folgeeinschränkungen oder Einflüssen auf die Zufriedenheit insgesamt 605 Patienten (63\%) einen Schmerz $(\mathrm{NRS} \geq 1$ ) angaben. 


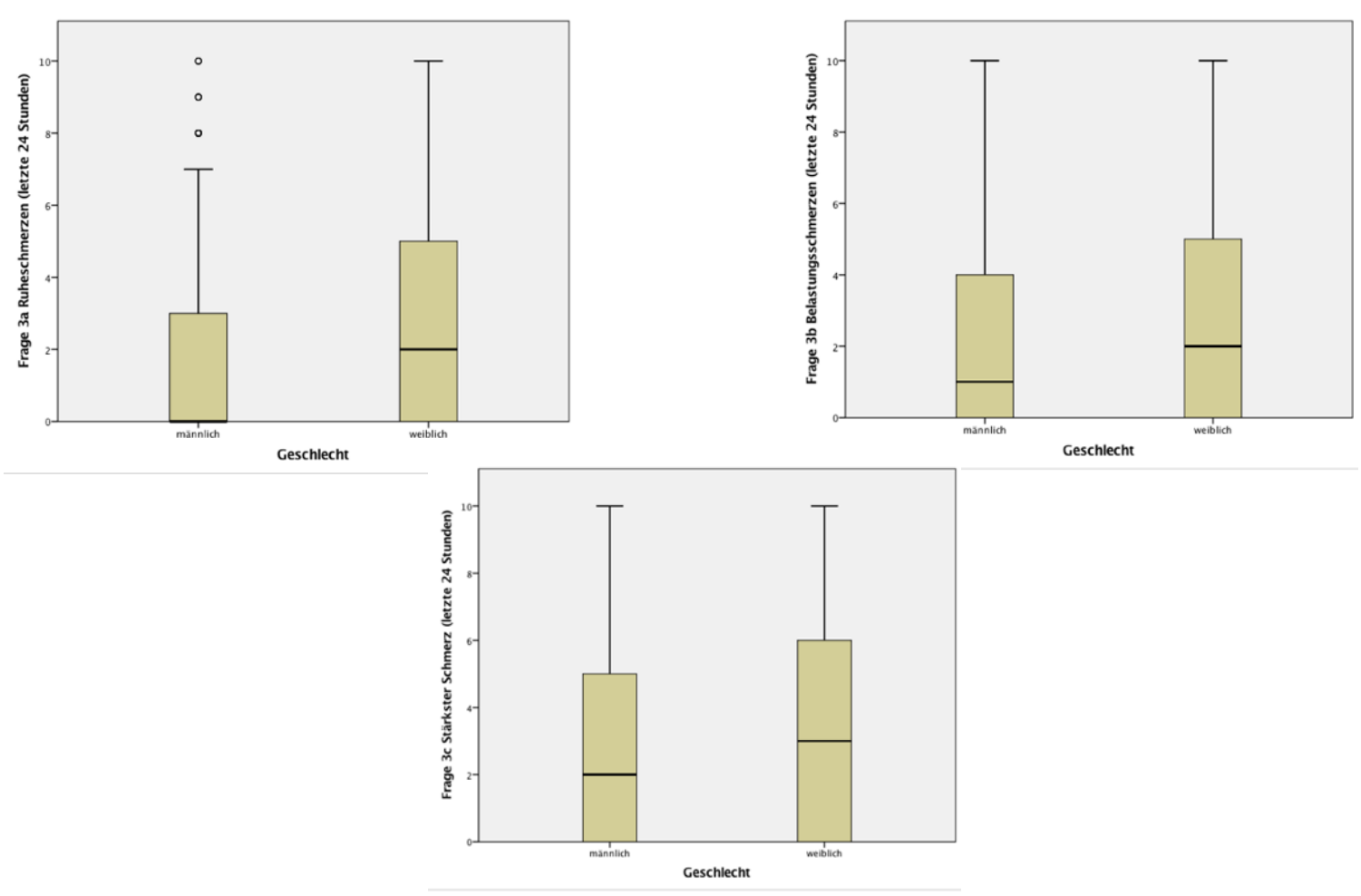

Abbildung 14: Geschlechterspezifische Darstellung für "Ruhe-, Belastungs- und stärkster Schmerz" in den vergangenen 24 Stunden vor der Befragung

\subsubsection{Frage 4: Funktionelle Beeinträchtigungen}

Bei den Fragen 4 und 5 wurde vor allem in Interviews immer wieder deutlich, dass die Patienten Schwierigkeiten hatten, die erfragten Beeinträchtigungen rein auf möglicherweise erlebte Schmerzen zurückzuführen. Vielfach wurden sie eher als Symptome einer Grunderkrankung oder des allgemeinen Krankenhausaufenthaltes interpretiert. Es ist nicht auszuschließen, dass diese Schwierigkeiten bei allen befragten Patienten, einschließlich derer, die den Fragebogen selbstständig ausfüllten, vorlagen. Die Ergebnisse sind daher kritisch zu betrachten, da unklar ist, ob hier trotz ausführlicher Erklärungen zu Beginn, wirklich eine reine Schmerzursache der mögliche Auslöser für die angegebenen Beschwerden war.

In Frage 4 wurden zusammengefasst funktionelle Beeinträchtigungen im Alltag durch Schmerzen in den vergangenen 24 Stunden abgefragt. Mit insgesamt 40\% der Patienten stellten die Beeinträchtigungen bei Bewegungen (Frage 4a) die größte Gruppe dar. 26\% der Patienten gaben eine negative Beeinflussung ihrer Gemütslage (Frage 4d) und 24\% einen schlechten Schlaf (Frage 4c) durch die empfundenen Schmerzen an. Lediglich 13\% fühlten sich beim Husten oder tiefen Luftholen (Frage 4b) beeinträchtigt. 
Wurden diese Daten nur auf die 605 Patienten reduziert, die in der Indikatorfrage 3c einen NRS-Wert $\geq 1$ angaben, ergaben sich für diese "eigentlichen" Patienten mit Schmerzen jeweils höhere Anteile von 58\% (Frage 4a), 18\% (Frage 4b), 36\% (Frage 4c) und 39\% (Frage 4d).

\subsubsection{Frage 5: Therapiebedingte Nebenwirkungen}

Diese Frage zielte auf den Allgemeinzustand der Patienten innerhalb der vergangenen 24 Stunden ab, der zum Beispiel durch Nebenwirkungen einer Schmerzmedikation beeinflusst worden sein konnte.

So fühlten sich 51\% der Patienten in den abgelaufenen 24 Stunden sehr müde (Frage 5a). Insgesamt gaben auch 14\% an unter Übelkeit (Frage 5b) gelitten zu haben, 27\% kreuzten dies auch bei Schwindel (Frage 5c) und 16\% bei einer Obstipation (Frage 5d) an. Die Werte in Bezug auf die Patienten mit Schmerzen (NRS Maximalschmerz >1) variierten hiervon analog zu Frage 4 ebenfalls etwas nach oben auf 62\% (Frage 5a), 17\% (Frage 5b), 32\% (Frage 5c) und 19\% in Frage 5d. Ein sicherer Bezug zur Medikation ließ sich aus den Fragen nicht ableiten. Allenfalls können diese Daten einen Vergleich der Häufigkeit von Symptomen bei Patienten mit und ohne Schmerzen, bzw. mit und ohne Medikation ermöglichen.

\subsubsection{Frage 6, 7 und 8: Patienteninformation und Zufriedenheit}

Insgesamt gaben vom gesamten Patientenkollektiv lediglich 350 von 958 Patienten an, über Möglichkeiten einer Schmerztherapie informiert worden zu sein. Dies ist ein Anteil von 37\%. Bei differenzierter Betrachtung auf die 605 Patienten mit Schmerzen während des Aufenthaltes reduziert, ergaben sich hier immerhin 44\%, die Informationen erhielten. Interessanterweise gaben aber auch nur $13 \%$ aller Patienten $(n=126)$ und $19 \%$ der Patienten mit Schmerzen an, dass sie in dieser Zeit mehr Schmerzmitteln gewünscht hätten. Die Zufriedenheit mit dem Ergebnis der erhaltenen Schmerztherapie konnten die Patienten ebenfalls auf einer NRS von 0 (völlig unzufrieden) bis 10 (sehr zufrieden) angeben. Bei der durchgeführten Auswertung wurde sich auf die Patienten mit vorbestehenden chronischen Schmerzen, auf Patienten mit einem akuten Schmerz bei der Klinikaufnahme und auf die bereits definierten Patienten mit einer Angabe zum akuten Maximalschmerz 24 Stunden vor der Befragung (Indikatorfrage 3c; NRS $\geq 1$ ) beschränkt (Abbildung 15). 
Es zeigte sich, dass von den 393 Patienten mit chronischen Schmerzen (Kapitel 3.2.1) insgesamt 315 eine Aussage zur Zufriedenheit mit der Schmerztherapie tätigten. 11 Patienten machten hier keine Angabe und für 67 kam subjektiv keine Therapie zur Anwendung. 10 Patienten machten hier jedoch auch doppelte und damit widersprüchliche Angaben, indem sie eine Zufriedenheitsangabe abgegeben haben und danach zusätzlich ankreuzten keine Therapie erhalten zu haben.

Insgesamt gab die große Mehrheit der Patienten mit chronischen Schmerzen zur Zufriedenheit Werte von NRS 5 und besser an (66\%), der Median lag hier bei 8 (5 - 10).

Wurden nur die Patienten betrachtet, die eine Aussage über einen Schmerz bei der Aufnahme (NRS $\geq 1$ ) machten, so gaben hier 69\% von 452 Patienten eine Zufriedenheit über NRS 5 an, der Median war hier ebenfalls $8(5-10)$.

Auch in diesem Fall gab es von 13 Patienten widersprüchliche Angaben zwischen einer Zufriedenheit und dem subjektiven Gefühl keine Therapie erhalten zu haben.

Von den 605 Patienten, die in der Indikatorfrage einen Schmerz mit NRS $\geq 1$ angaben, machten insgesamt 491 Gebrauch von einer Angabe zur Zufriedenheit. Diese war im Median 8 (5 - 10). 15 Patienten nutzten sie nicht und 99 gaben an keine Therapie benötigt zu haben. Hierzu gab es wie bei den Auswertungen zuvor schon ebenfalls 12 doppelte Angaben.

Den Befragenden wurde insgesamt sehr häufig zurückgemeldet, dass manche Patienten keine Schmerzen angaben, obwohl sie z. T. sehr wohl Schmerzen hatten, aber entsprechend keine Schmerztherapie erhielten oder einforderten. Die aktuelle Formulierung der Auswahlmöglichkeit in Frage 8 (Abbildung 6) kann diesen Tatbestand jedoch so nicht abbilden, wodurch es hier immer wieder zu Missverständnissen kam. Eine Möglichkeit wäre hier zu ergänzen, dass zwar Schmerzen bestehen, aber keine entsprechende Therapie gewünscht ist (weil der Schmerz nicht als beeinträchtigend eingeschätzt wird oder als nicht relevant für die aktuelle Behandlung. Das wurde auch darin deutlich, dass es in diesem Zusammenhang zu doppelten bzw. widersprüchlichen Angaben kam. Ebenfalls wurde den Befragenden häufig zurückgemeldet, dass die Patienten die NRS zur Zufriedenheit falsch anwendeten, weil die positive/negative Zahlenzuordnung in der NRS umgekehrt zur Schmerzskala war. 
Interessanterweise bestanden auch widersprüchliche Ergebnisse zwischen der Antwortmöglichkeit „keine Schmerzen“ in Frage 8 (Wunsch nach Schmerzmedikation) und allen vorangegangenen Fragen. Auch wenn die meisten Patienten hier inhaltlich korrekt antworteten, gab es auch Patienten die einen Wunsch nach mehr Schmerzmitteln äußerten, obwohl sie am Ende angaben, nie unter Schmerzen gelitten zu haben.
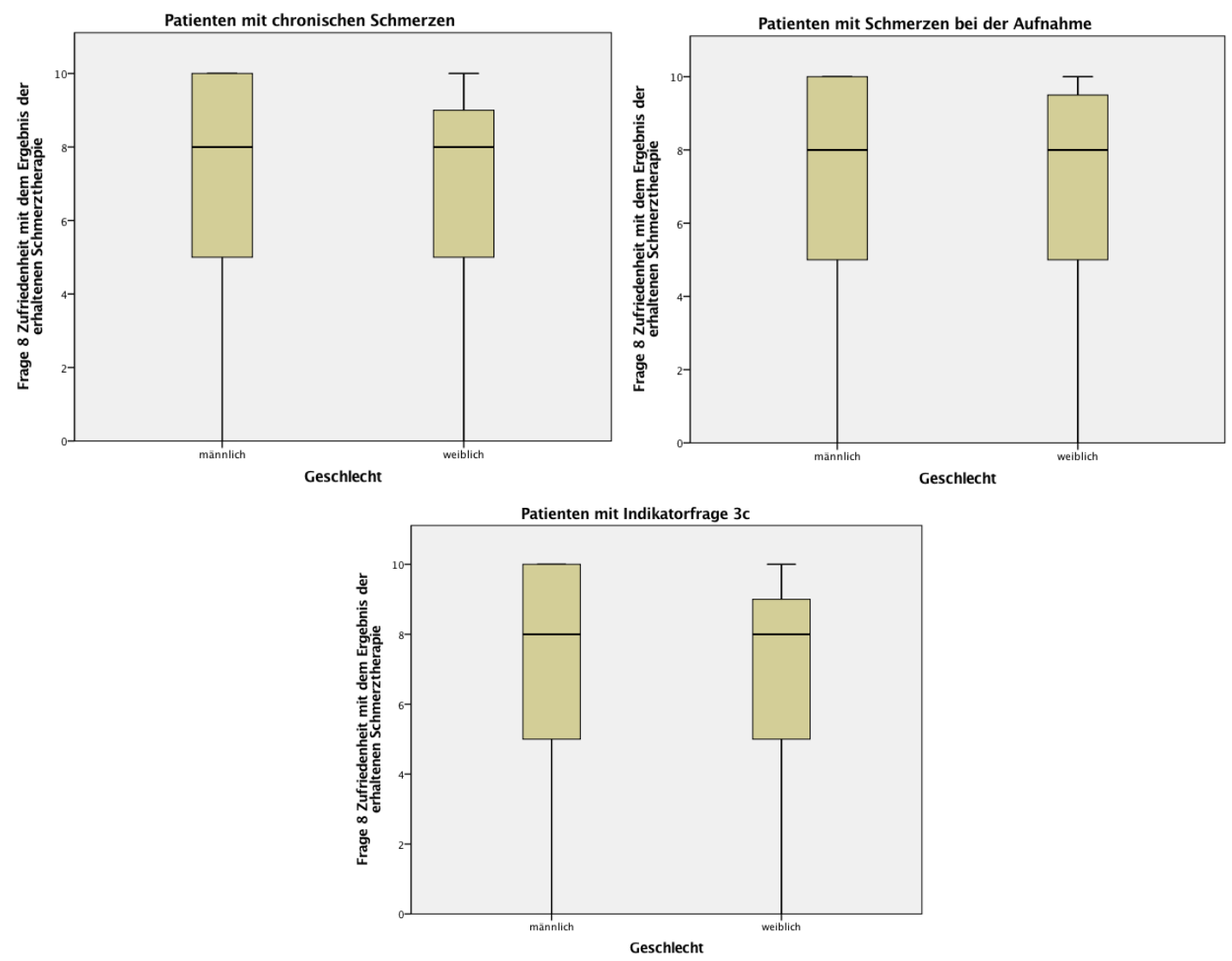

Abbildung 15: Angaben zur Patientenzufriedenheit mittels NRS, jeweils bei Patienten mit chronischen Schmerzen, mit Schmerzen bei der Aufnahme und mit einer Schmerzangabe in der Indikatorfrage $3 \mathrm{c}$

\subsubsection{Interviewrate}

Der Großteil der Patienten konnte den Fragebogen selbst ausfüllen, allerdings mit den bereits angesprochenen zum Teil widersprüchlichen Angaben. Bei insgesamt 310 (33\%) Patienten wurde der Fragebogen in Form eines Interviews durchgeführt. Die Geschlechterverteilung unterschied sich hier mit 160 Männern zu 147 Frauen nicht. 


\subsubsection{Subjektive Einschätzungen der Befragenden}

Die ersten Einschätzungen zum Befragungsmodell wurden in Göttingen nach der Voruntersuchung in Testphase 1 und mit den ersten beiden Testphasen zu Beginn dieser Promotionsarbeit getätigt. Nach dem Abschluss der letzten Projektphase zeigten sich in Rücksprache mit den algesiologischen Fachassistenten an allen nun hinzugekommenen Befragungsorten innerhalb des Ergebnisfragebogens wie schon in den vorangegangenen Testphasen übereinstimmende subjektive Schwierigkeiten und Schwächen. Standortübergreifend bestand der Eindruck, dass vor allem Patienten im höheren Lebensalter oder mit einer hohen Komorbidität häufiger überfordert waren. Eine formale Erfassung die Eindrücke erfolgt nicht.

Schwierigkeiten bei der Anwendung der Prozessparameter bestanden nicht. Gleichzeitig waren alle Beteiligten subjektiv von einer generellen Anwendbarkeit überzeugt.

\subsection{Projektphase: Quantitative Auswertung der Prozessparameter}

\subsubsection{Haupt- und Nebendiagnosen}

Zur einfacheren Erfassung der Hauptdiagnose wurde den einzelnen Organsystemen (Tabelle 6) innerhalb der Wortdiagnoseliste eine übergeordnete Vorauswahl zur Verfügung gestellt. In der tabellarischen Auflistung wurden alle Diagnosen von „nicht eingeschlossenen" Patienten und wenn keine Diagnose angekreuzt wurde $(n=9)$ herausgenommen. An den drei verschiedenen Befragungsstandorten waren die am häufigsten betroffenen Organsysteme das Nervensystem (27\%) und die Haut (15\%). Mit knapp 26\% nahm auch die Gruppe der bisher nicht aufgelisteten, aber klar definierten „sonstigen Hauptdiagnosen" einen großen Anteil ein und wies auf die Notwendigkeit hin die Wortdiagnoseliste weiter anzupassen und den Einsatz als Benchmarking-Faktor weiter zu prüfen.

Dieses Verteilungsmuster wurde aber auch durchaus aufgrund der unterschiedlichen Repräsentanz der verschiedenen Fachgebiete induziert, da einige Fachdisziplinen erst im laufenden Befragungszeitraum an dem Projekt beteiligt wurden.

Herzerkrankungen (8\%), sowie onkologische Hauptdiagnosen aus dem Kreise der Malignen Neoplasien (7\%) gehörten ebenfalls zu den häufiger vertretenen Hauptdiagnosen. $6 \%$ der Hauptdiagnosen waren am Befragungstag noch "nicht erhebbar" und wurden nur als die aktuell vorliegende Symptomatik erfasst. Es lässt sich somit festhalten, dass mit 
der aktuellen Wortdiagnoseliste am Befragungstag trotz aller Heterogenität in 94\% der Diagnosen eine klar definierte Hauptdiagnose zuzuordnen war, auch wenn nicht alle bereits namentlich hinterlegt waren und somit durch den Befragenden unter "Sonstiges" ergänzt werden mussten.

Sehr selten waren mit jeweils weniger als 1\% Erkrankungen der Organsysteme Stoffwechsel/Hormone, Niere, Infektionen und Blut.

Detailliert betrachtet (Tabelle 7) war mit anteilig knapp 9\% die häufigste Hauptdiagnose der Schlaganfall. Von den weiteren Vorgaben der Wortdiagnoseliste überschritt sonst keine mehr die 5\%-Grenze. Nachfolgend wurden mit anteilig je 3\% Primäre Herzrhythmusstörungen, Transitorisch ischämische Attacken (TIA), Psoriasis, Multiple Sklerose und Sklerodermie und mit je 2\% der M. Parkinson, Maligne Neoplasien des Pankreas, sonstige Maligne Neoplasien, Ekzem, Epilepsie, Kopfschmerz, Polyneuropathie, Schwindel und Rheumatische Arthritis angegeben. Alle weiteren Hauptdiagnosen waren mit nur etwa anteilig 1\% vertreten und wurden zur besseren Übersicht aus der Tabelle herausgenommen.

Unter der Rubrik „sonstige Hauptdiagnose“ wurde mit 5\% am häufigsten eine gastrointestinale Blutung eingetragen, gefolgt mit 3\% die Stauungsdermatitis und mit je $2 \%$ die Amyothrophische Lateralsklerose (ALS), die chronisch-inflammatorische demyelinisierende Polyneuropathie (CIDP), die Leberzirrhose, Myasthenia gravis und Prurigo nodularis. Einen Sonderfall stellen hier die dermatologischen Hauptdiagnosen „Hyposensibilisierung gegen Wespen (3\%) oder Bienen (1\%)“ dar. In der Projektphase wurden diese Patienten einheitlich als „sonstige Hauptdiagnose“ gewertet, wobei hier sicherlich zu diskutieren wäre, ob besser die zugrunde liegende Allergie als Diagnose erfasst werden sollte. 
Tabelle 6: Zugehörigkeiten der jeweiligen Hauptdiagnosen zu den spezifischen Organsystemen

\begin{tabular}{|l|c|c|c|}
\hline Organsystem & $n$ & Prozent & Gültig (\%) \\
\hline Herz & 79 & 6,0 & 8,2 \\
\hline Kreislauf & 24 & 1,8 & 2,5 \\
\hline Magen/Darm & 17 & 1,3 & 1,8 \\
\hline Leber/Pankreas/Milz & 19 & 1,4 & 2,0 \\
\hline Stoffwechsel/Hormone & 5 & 0,4 & 0,5 \\
\hline Niere & 8 & 0,6 & 0,8 \\
\hline Lunge & 27 & 2,1 & 2,8 \\
\hline Nervensystem & 264 & 20,0 & 27,4 \\
\hline Haut & 141 & 10,7 & 14,6 \\
\hline Bewegungsapparat & 22 & 1,7 & 2,3 \\
\hline Infektion & 8 & 0,6 & 0,8 \\
\hline Blut & 1 & 0,1 & 0,1 \\
\hline Maligne Neoplasien & 70 & 5,3 & 7,3 \\
\hline Nicht erhebbar & 219 & 16,6 & 2,7 \\
\hline Sonstiges & 19,6 & \\
\hline
\end{tabular}


Tabelle 7: Übersicht der spezifischen Hauptdiagnosen >1\% Häufigkeit

\begin{tabular}{|c|c|c|c|}
\hline Diagnose & $\mathrm{n}$ & Prozent & Gültig (\%) \\
\hline ACS & 13 & 1,0 & 1,3 \\
\hline Arterielle Hypertonie & 11 & 0,8 & 1,1 \\
\hline Blasenbildende Autoimmundermatose & 14 & 1,1 & 1,5 \\
\hline COPD/obstruktive Lungenerkrankungen & 12 & 0,9 & 1,2 \\
\hline Dekompensierte Herzinsuffizienz & 14 & 1,1 & 1,5 \\
\hline Ekzem & 19 & 1,4 & 2,0 \\
\hline Epilepsie & 17 & 1,3 & 1,8 \\
\hline Erysipel & 13 & 1,0 & 1,3 \\
\hline KHK & 10 & 0,8 & 1,0 \\
\hline Kopfschmerz & 18 & 1,4 & 1,9 \\
\hline M. Parkinson & 23 & 1,7 & 2,4 \\
\hline Maligne Neoplasie Leber & 11 & 0,8 & 1,1 \\
\hline Maligne Neoplasie Pankreas & 20 & 1,5 & 2,1 \\
\hline Maligne Neoplasie Sonstiges & 21 & 1,6 & 2,2 \\
\hline Multiple Sklerose & 24 & 1,8 & 2,5 \\
\hline Polyneuropathie & 21 & 1,6 & 2,2 \\
\hline Primäre Rhythmusstörung & 31 & 2,4 & 3,2 \\
\hline Psoriasis & 27 & 2,1 & 2,8 \\
\hline Rheumatische Arthritis & 17 & 1,3 & 1,8 \\
\hline Schlaganfall & 84 & 6,4 & 8,7 \\
\hline Schwindel & 22 & 1,7 & 2,3 \\
\hline Sklerodermie & 24 & 1,8 & 2,5 \\
\hline Sonstiges & 249 & 18,9 & 25,8 \\
\hline Synkope & 12 & 0,9 & 1,2 \\
\hline TIA & 30 & 2,3 & 3,1 \\
\hline
\end{tabular}

\subsubsection{Schmerzrelevante Vormedikation}

In der schmerztherapeutisch wirksamen Vormedikation wurde zwischen Nicht-Opioiden, Opioiden und Ko-Analgetika unterschieden.

Die Wirkstoffgruppen mit einem eingesetzten Anteil von weniger als $5 \%$ wurden zur besseren Übersicht in der Auswertung der Vor- und Stationsmedikation vernachlässigt. 
Bereits 114 Patienten hatten in der Vormedikation einen Bedarf an Opioiden (43 Männer, 71 Frauen). Zudem erhielten insgesamt 170 Patienten ein Nicht-Opioid und 143 Patienten ein Ko-Analgetikum.

Zu den anteilig am häufigsten eingesetzten Medikamenten in der Vormedikation zählten in der Gruppe der Nicht-Opioide mit knapp 61\% Metamizol und mit 22\% Ibuprofen. Diclofenac (7\%) und Paracetamol (5\%) folgten hier mit sehr großem Abstand. Nur einer von 220 Patienten nahm ASS in der analgetisch-wirksamen Dosierung von 500 mg p. o. ein.

Selektive COX-2-Inhibitoren wie beispielsweise Celecoxib oder Etoricoxib waren jeweils nur vier Patienten verordnet worden.

Im Bereich der Opioide fand sich ein ähnliches, aber nicht so deutlich aufgetrenntes Verteilungsmuster. Mit knapp 40\% erhielten die Patienten in der Vormedikation am häufigsten ein niedrigpotentes Opioid wie Tilidin (29\%) oder Tramadol (11\%).

Von den höher- bis hochpotenten Opioiden wurden vor allem Morphin (9\%), Oxycodon (11\%), Oxycodon kombiniert mit Naloxon (14\%) und Fentanyl (14\%) eingenommen.

Hydromorphon war mit knapp 6\% das letzte Opioid mit einem Anteil von größer 5\%.

Die am häufigsten eingesetzten Ko-Analgetika waren die Antikonvulsiva, mit knapp 32\% Pregabalin und mit 13\% Gabapentin. Aus der Gruppe der Antidepressiva fanden Amitryptilin (15\%), Citalopram (13\%) und Duloxetin (8\%) häufig Anwendung.

Das Benzodiazepin Lorazepam (6\%) als Anxiolytikum war ein weiteres Medikament mit einem Anteil von größer 5\%.

Aktuell nicht aufgelistete analgetisch bzw. koanalgetisch wirksame Medikamente wurden unter der Rubrik „andere“ hinzugefügt. Mit Abstand am häufigsten wurde hier Mirtazapin (36\%) eingesetzt. Darauf folgten Venlafaxin (15\%), Baclofen (14\%), Quensyl (10\%) und Topiramat und Valproinsäure (beide 7\%). Die in der zweiten Testphase festgelegten „anderen Wirkstoffe" werden vor der klinischen Einführung von QUIKS in die Medikamentenliste fest aufgenommen. Allerdings ist für viele dieser Wirkstoffe eine Zuordnung der Indikation als analgetisch nicht möglich.

\subsubsection{Schmerzrelevante Stationsmedikation}

Während des Klinikaufenthalts erhielten an den drei verschiedenen Befragungsorten insgesamt 150 Patienten Opioide. Im Vergleich zur Vormedikation waren es lediglich 7 männliche und 14 weibliche Patienten mehr. 
Mit anteilig 65\% wurde auch auf den Stationen Metamizol als das mit weitem Abstand häufigste Nicht-Opioid eingesetzt. Ibuprofen wurde mit 15\% etwas weniger, dafür Paracetamol mit 13\% etwas häufiger verordnet als in der Vormedikation. Die Verhältnisse bei den übrigen Nicht-Opioiden waren zur Vormedikation nahezu unverändert.

Auch die Verteilung der Opioide entsprach weitgehend der Anwendung in der Vormedikation.

Tilidin stellte als niedrigpotentes Opioid weiterhin mit anteilig 20\% das am häufigsten verabreichte Opioid dar. Anstelle von Fentanyl (13\%) folgten aber vorab Oxycodon (15\%), Tramadol (14\%) und Oxycodon kombiniert mit Naloxon mit ebenfalls 13\%.

Abschließend komplettierten Hydromorphon (7\%) und Morphin (8\%) die Gruppe der Opioide innerhalb der Stationsmedikation mit einem Anteil größer 5\%. Diese Verteilung reflektiert vermutlich die typischen Standards in den teilnehmenden Kliniken.

Bei den Ko-Analgetika war die Verteilung ebenfalls nahezu unverändert zur Vormedikation. Die Antikonvulsiva Pregabalin (34\%) und Gabapentin (13\%) wurden weiterhin bevorzugt eingesetzt. Aus der Gruppe der Antidepressiva wurde im Vergleich zur Vormedikation Citalopram (14\%) häufiger dem Amitryptilin (11\%) vorgezogen. Gleiches galt für Lorazepam (8\%) und Duloxetin (7\%).

Innerhalb der Rubrik „andere“ variierte die Verteilung ebenfalls nicht. Das Spektrum der verordneten Medikamente war zwar erweitert, aber die Anzahl der Verordnungen blieb nahezu durchgehend unter 5\%. Mirtazapin stellte mit 41\% erneut den häufigsten Wirkstoff dar, der mit großem Abstand von Baclofen (11\%) gefolgt wurde.

Quensyl, Topiramat und Valproinsäure waren die einzigen weiteren Medikamente knapp oberhalb der 5\%-Grenze. Insgesamt weisen die Daten zur Medikation nicht auf eine relevante Veränderung im Rahmen des Aufenthaltes hin, wobei allerdings Änderungen der Dosierung nicht erfasst wurden.

Abschließend konnte festgehalten werden, dass bei keinem der befragten nicht operierten Patienten ein spezielles Schmerztherapieverfahren (z. B. PCA-Pumpe) eingesetzt wurde.

\subsubsection{Therapieanordnung}

Bei der Frage nach einer individuellen Therapieanordnung wurde zusätzlich nochmals eine Unterteilung nach einer Basis- und Bedarfsmedikation durchgeführt. 
Hier stellte sich in einigen Fällen die Frage, wie damit verfahren wird, wenn ein Patient keine nichtsteroidalen Antirheumatika (NSAR) oder Opioide erhielt, dafür aber eine analgetisch wirksame Dosis von z. B. Pregabalin im Rahmen eines neuropathischen Schmerzes. Es wurden deshalb noch einmal die SOP von QUIPS als Vergleich herangezogen. Dort zeigte sich, dass diese umfangreiche Unterscheidung dort keine Rolle spielt und sich dort rein auf eine Handlungsfähigkeit der Pflegekräfte auf Station bezieht. Im dortigen Wortlaut sieht die Therapieanordnung eine individuelle Bedarfsmedikation vor. Eine zukünftige Unterscheidung zwischen einer individuellen Therapieanordnung und der Erfassung einer Bedarfsmedikation stellte sich daher auch voraussichtlich als doppelt und unnötig dar. Für die Projektphase wurde daraufhin einheitlich festgelegt, dass diesbezüglich nur NSAR und Opioide erfasst werden.

Unter dieser Berücksichtigung wurde auf den Stationen insgesamt 23\% der Patienten eine Basisanalgesie verabreicht und weniger als die Hälfte (48\%) hatten eine angeordnete Bedarfsmedikation. Bei Patienten mit einem in Frage 1a angegebenen chronischen Schmerz waren diese mit 34\% und 54\% etwas häufiger angeordnet.

\subsubsection{Schmerzdokumentation}

Ein Aspekt der Test- und Projektphasen war die mangelhafte Dokumentation der Schmerzen auf den jeweiligen Stationen, sowohl auf ärztlicher Seite, als auch im Bereich der Pflege. Während der Befragungen wurde jede mögliche Form einer Schmerzdokumentation (z. B. in Textform während der Visite oder mittels NRS) von den behandelnden Ärzten und/oder Pflegekräften gewertet. Alle klinischen Dokumentationssysteme, ob elektronisch oder in einer handschriftlichen Patientenkurve, hätten eine Schmerzerfassung grundsätzlich ermöglicht.

In der Projektphase wurde an allen drei Befragungsorten in den vergangenen 24 Stunden vor der Befragung nur bei 18\% der Patienten eine Erfassung der Schmerzen durchgeführt. In vielen Fällen wurde auch nur eine sehr lückenhafte Schmerzerfassung dokumentiert, die durch den Befragenden nur dann mit „ja“ erfasst wurde, wenn sie am Tag vor der Befragung stattgefunden hat. Auffällig war zudem das sehr viele Patienten eine Bedarfsmedikation erhalten haben und diese auch ordnungsgemäß dokumentiert wurde, obwohl kein nachvollziehbarer Schmerz (z. B. mittels NRS) in der Kurve ersichtlich war, der 
die Gabe einer Bedarfsmedikation rechtfertigte. Zudem wurde nach der Gabe einer Bedarfsmedikation in keinem Fall eine erneute Schmerzdokumentation durchgeführt, ob die verabreichte Schmerztherapie eine ausreichende Wirkung hatte.

\subsection{Qualitative Auswertung des Erhebungsinstruments}

Nach Abschluss der endgültigen Projektphase wurde ein erster Versuch der qualitativen Bewertung des neuen Erhebungsfragebogens durchgeführt. Zur besseren Vergleichbarkeit der Studie wurde hier eine mit Meißner et al. (2008) und Rothaug et al. (2013) vergleichbare Methodik angewendet. Aufgrund der hohen Variablenzahl im Datensatz wurde zur Variablenreduktion die explorative Faktorenanalyse und Hauptkomponentenanalyse mit Promax-Rotation ausgewählt. Zu Beginn der Auswertung wurde die Eignung dieser Herangehensweise durch das Kaiser-Meyer-Olkin-Kriterium (KMO-Wert = $0,856)$ und dem Bartlett-Test ( $<<0,001)$ gerechtfertigt (Abbildung 16).

\begin{tabular}{|c|c|c|}
\hline \multicolumn{3}{|c|}{ KMO- und Bartlett-Test } \\
\hline \multicolumn{2}{|c|}{ Maß der Stichprobeneignung nach Kaiser-Meyer-Olkin } & 0,856 \\
\hline \multirow[t]{3}{*}{ Bartlett-Test auf Sphärizität } & Ungefähres Chi-Quadrat & 2234,301 \\
\hline & Df & 120 \\
\hline & Signifikanz nach Bartlett & 0,000 \\
\hline
\end{tabular}

Abbildung 16: KMO- und Bartlett-Test: 3 Faktoren

Die Faktorenzahl wurde in Anlehnung an die Ergebnisse von Rothaug et al. 2013 zunächst für die Fragen 2b bis 8 auf drei Faktoren festgelegt. Nach dieser festen Vorgabe konnten 43,6\% der Varianz erklärt werden (Tabelle 9). Wenn rein das Kaiser-Guttmann-Kriterium zugrunde gelegt wurde, nach dem nur Eigenwerte $>1,0$ berücksichtigt werden dürfen, ergab die Faktorenanalyse eine vierfaktorielle Lösung, die 50,2\% der Varianz erklärte. Als entsprechende Gegenkontrolle wurde der Scree-Test (Ellenbogenkriterium) durchgeführt. Der daraus resultierende Screeplot machte jedoch auch graphisch deutlich, dass der vierte Faktor (Eigenwert 1,044) nur gering oberhalb des Niveaus der Zufallskorrelation lag (Abbildung 17). Aufgrund dessen und der besseren Vergleichsmöglichkeiten mit Rothaug et al. 2013 wurde die dreifaktorielle Lösung beibehalten. Die interne Konsistenz 
aller eingeschlossenen Fragen wurde mittels Cronbachs $\alpha$ überprüft und zeigte einen statistisch „noch grenzwertig akzeptablen“ Wert von 0,70 (Tabelle 8). Aufgrund des Cronbachs $\alpha$, des Screeplots und anschließender theoretischer Überlegungen wurde eine Problemanalyse durchgeführt, in der sich die sehr hohe Heterogenität der Patientengruppe als problematischer Faktor herausstellte.

Viele der befragten Patienten hatten bei der Beantwortung besonders die Frage 8 mit Angaben zur Zufriedenheit mit der Schmerztherapie nicht beantwortet, wodurch die Anzahl der für die Faktorenanalyse einzuschließenden Fälle ( $n=553$ von 973 Zustimmungen; Tabelle 11) stark reduziert wurde.

In diesem Zusammenhang wurden auch noch einmal die Rückmeldungen der Patienten deutlich, denen es schwer fiel eine Aussage über die Zufriedenheit zu treffen bzw. diese mit dem aktuellen Fragebogen passend angeben zu können, wenn sie keine Schmerztherapie (auch bei ggfs. bestehenden Schmerzen) benötigten oder nicht in Anspruch genommen hatten.

Tabelle 8: Berechnung des Cronbachs $\alpha$ (im Text gerundete Werte)

\begin{tabular}{|l|c|c|c|}
\hline Items & $n$ & Anzahl der Items & Cronbachs $\alpha$ \\
\hline Alle Fragen: Frage 2b bis 8 & 553 & 16 & 0,695 \\
\hline Fragen 2b bis 5d & 911 & 13 & 0,818 \\
\hline $\begin{array}{l}\text { Subskala 1: „Schmerzintensität und funktionellen Beein- } \\
\text { trächtigung“ }\end{array}$ & 926 & 9 & 0,836 \\
\hline $\begin{array}{l}\text { Subskala 2: „therapiebedingten Auswirkungen“ } \\
\text { Hypothetische Subskala: Nur die NRS-Fragen 2 und 3 }\end{array}$ & 956 & 4 & 0,484 \\
\hline Hypothetische Subskala: Nur die binären Fragen 4 und 5 & 940 & 5 & 0,888 \\
\hline
\end{tabular}

Vielfach wurde auch zurückgemeldet, dass hier bei den Patienten eine unterschiedliche Wahrnehmung bzw. sogar eine Art akzeptierte Voraussetzung, dass Schmerz bei einem Krankenhausaufenthalt zu erwarten ist. Trotz der inhaltlichen Nähe trug die Frage 7 mit dem Wunsch nach mehr Schmerzmitteln keine hohe Faktorenladung $(0,274)$ auf den Faktor mit der Patienteninformation $(0,719)$ und Patientenzufriedenheit $(0,720)$. Auch besteht für diese Fragen für QUIKS eine andere Situation als im Rahmen einer perioperativen Schmerztherapie, da nur ein Teil der Patienten Schmerzen hat. 
Als Lösung wurde daher eine erneute Faktorenanalyse (Frage $2 b$ bis $5 d$ ) ohne die betreffenden Fragen zur Patienteninformation und Patientenzufriedenheit durchgeführt, wodurch die einzuschließenden Fälle um ein Vielfaches anstiegen ( $n=911$ vs. 533; Tabelle 12) und der Screeplot etwas deutlicher wurde (Abbildung 18). Die Faktorenladungen ließen sich verbessert zuordnen und es ergaben sich letztlich nach der Eigenwert-Regel ohne feste Vorgabe nur zwei Faktoren mit einer erklärten Varianz von 48,7\% (Tabelle 10). Diese Faktoren konnten den Subskalen der „Schmerzintensität und funktionellen Beeinträchtigung“ und den „therapiebedingten Auswirkungen“ zugeordnet werden. Die Frage $4 \mathrm{~b}$ zur schmerzhaften Beeinflussung von Husten oder tiefem Luftholen zeigte für beide Faktoren eine auffällig niedrige Querladung $(<0,4)$ und konnte somit keinem der beiden Faktoren eindeutig zugeordnet werden.

Die interne Konsistenz verbesserte sich in der Folge für die Gesamtzahl der nun eingeschlossenen Fragen auf ein „gutes" Cronbachs $\alpha$ von 0,82. Für die einzelnen Subskalen zeigten sich hier „zufriedenstellende“ 0,84 für die „Schmerzintensitäten und schmerzbedingten Beeinträchtigung“ und nur „inakzeptable“ 0,48 für die „therapiebedingten Auswirkungen“ im Vergleich zu den Werten von Rothaug et al. (2013). Möglicherweise wurde bei der Beantwortung hier deutlich, dass einige Patienten fälschlicherweise Symptome oder Einschränkungen angaben, obwohl sie diese schmerzunabhängig empfunden hatten oder das im Kontext der konservativen Medizin diese Zuordnung heterogener ist. Eine jedoch ähnliche inhaltliche Konsistenz ließe sich aber theoretisch abbilden, wenn unabhängig von der Faktorenanalyse die NRS-Fragen 2 und 3 als eigene Subskala (Cronbachs $\alpha=0,89$ ) mit den dichotomen Fragen 4 und 5 als weitere Subskala (Cronbachs $\alpha=$ 0,63 ) berechnet würden (Tabelle 8). 


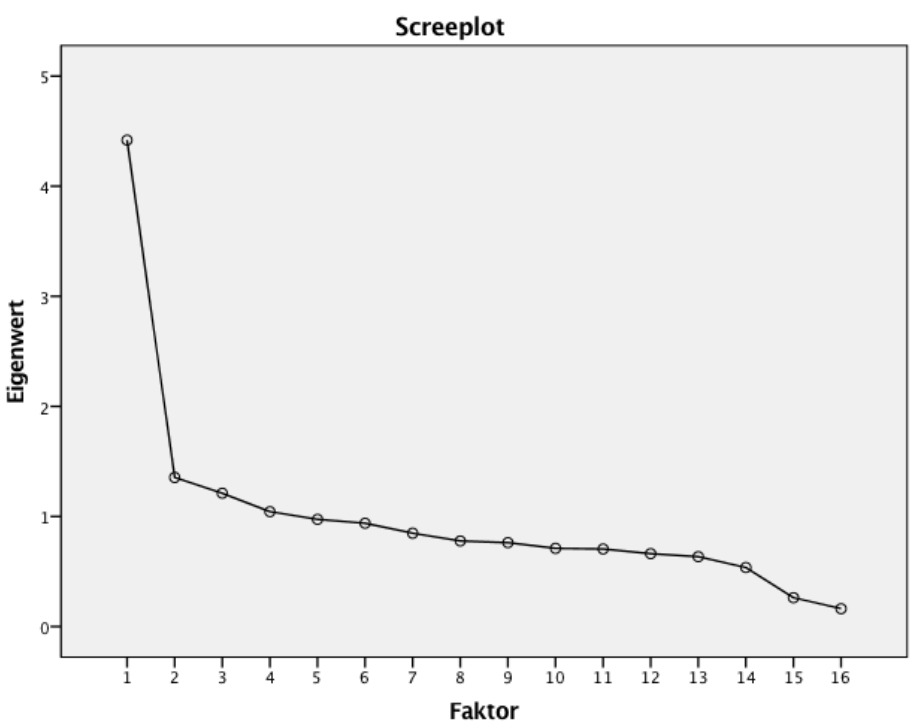

Abbildung 17: Screeplot vor Problemanalyse

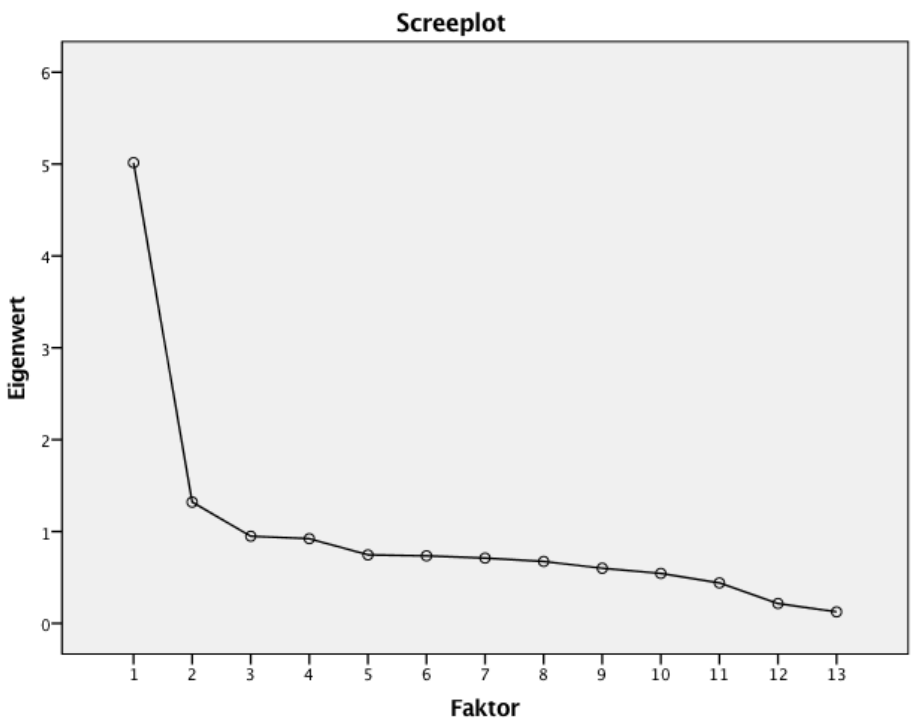

Abbildung 18: Screeplot nach Problemanalyse 
Tabelle 9: Erklärte Varianz vor der Problemanalyse

\begin{tabular}{|c|c|c|c|c|c|c|c|}
\hline \multirow[b]{2}{*}{ Faktoren } & \multicolumn{3}{|c|}{ Anfängliche Eigenwerte } & \multicolumn{3}{|c|}{$\begin{array}{l}\text { Summen von quadrierten Faktorladun- } \\
\text { gen für Extraktion }\end{array}$} & \multirow{2}{*}{$\begin{array}{l}\text { Rotierte Summe der } \\
\text { quadrierten Ladungen } \\
\text { Gesamt }\end{array}$} \\
\hline & Gesamt & $\%$ der Varianz & Kumulierte \% & Gesamt & $\%$ der Varianz & Kumulierte \% & \\
\hline 1 & 4,418 & 27,615 & 27,615 & 4,418 & 27,615 & 27,615 & 4,313 \\
\hline 2 & 1,355 & 8,467 & 36,082 & 1,355 & 8,467 & 36,082 & 2,148 \\
\hline 3 & 1,211 & 7,567 & 43,649 & 1,211 & 7,567 & 43,649 & 1,243 \\
\hline 4 & 1,044 & 6,524 & 50,174 & & & & \\
\hline 5 & 0,974 & 6,086 & 56,260 & & & & \\
\hline 6 & 0,938 & 5,864 & 62,124 & & & & \\
\hline 7 & 0,848 & 5,298 & 67,422 & & & & \\
\hline 8 & 0,778 & 4,860 & 72,283 & & & & \\
\hline 9 & 0,762 & 4,764 & 77,047 & & & & \\
\hline 10 & 0,710 & 4,440 & 81,487 & & & & \\
\hline 11 & 0,704 & 4,403 & 85,890 & & & & \\
\hline 12 & 0,662 & 4,138 & 90,028 & & & & \\
\hline 13 & 0,635 & 3,967 & 93,995 & & & & \\
\hline 14 & 0,536 & 3,351 & 97,346 & & & & \\
\hline 15 & 0,262 & 1,639 & 98,985 & & & & \\
\hline 16 & 0,162 & 1,015 & 100,000 & & & & \\
\hline \multicolumn{8}{|c|}{ Extraktionsmethode: Hauptkomponentenanalyse. } \\
\hline $\begin{array}{l}\text { a. Wenn K } \\
\text { Gesamtva }\end{array}$ & $\begin{array}{l}\text { mponent } \\
\text { anz zu erl }\end{array}$ & $\begin{array}{l}\text { en korreliert sin } \\
\text { halten. }\end{array}$ & d, können die S & Immen $d$ & er quadrierten $\mathrm{L}$ & -adungen nicht & ddiert werden, um eine \\
\hline
\end{tabular}

Tabelle 10: Erklärte Varianz nach der Problemanalyse

\begin{tabular}{|c|c|c|c|c|c|c|c|}
\hline \multirow[b]{2}{*}{ Faktoren } & \multicolumn{3}{|c|}{ Anfängliche Eigenwerte } & \multicolumn{3}{|c|}{$\begin{array}{l}\text { Summen von quadrierten Faktorladun- } \\
\text { gen für Extraktion }\end{array}$} & \multirow{2}{*}{$\begin{array}{c}\text { Rotierte Summe der } \\
\text { quadrierten Ladungen } \\
\text { Gesamt }\end{array}$} \\
\hline & Gesamt & $\%$ der Varianz & Kumulierte \% & Gesamt & \% der Varianz & Kumulierte \% & \\
\hline 1 & 5,015 & 38,578 & 38,578 & 5,015 & 38,578 & 38,578 & 4,921 \\
\hline 2 & 1,321 & 10,158 & 48,736 & 1,321 & 10,158 & 48,736 & 2,213 \\
\hline 3 & 0,949 & 7,302 & 56,037 & & & & \\
\hline 4 & 0,922 & 7,092 & 63,130 & & & & \\
\hline 5 & 0,746 & 5,739 & 68,869 & & & & \\
\hline 6 & 0,735 & 5,656 & 74,525 & & & & \\
\hline 7 & 0,711 & 5,473 & 79,998 & & & & \\
\hline 8 & 0,674 & 5,183 & 85,181 & & & & \\
\hline 9 & 0,600 & 4,616 & 89,797 & & & & \\
\hline 10 & 0,544 & 4,184 & 93,980 & & & & \\
\hline 11 & 0,441 & 3,389 & 97,369 & & & & \\
\hline 12 & 0,216 & 1,664 & 99,033 & & & & \\
\hline 13 & 0,126 & 0,967 & 100,000 & & & & \\
\hline \multicolumn{8}{|c|}{ Extraktionsmethode: Hauptkomponentenanalyse. } \\
\hline
\end{tabular}


Tabelle 11: Deskriptive Statistik und Faktorenanalyse mit drei festgelegten Faktoren

\begin{tabular}{|c|c|c|c|c|c|c|}
\hline & \multicolumn{3}{|c|}{ Faktoren } & \multicolumn{3}{|c|}{ Deskriptive Statistik } \\
\hline & 1 & 2 & 3 & Mittelwert & Standardabweichung & $\mathrm{n}$ \\
\hline $\begin{array}{l}\text { Frage } 2 b \text { Schmerzstärke bei Krankenhaus- } \\
\text { aufnahme }\end{array}$ & 0,629 & 0,184 & 0,122 & 4,44 & 3,239 & 553 \\
\hline $\begin{array}{l}\text { Frage 2c Stärkster Schmerz seit der Kran- } \\
\text { kenhausaufnahme }\end{array}$ & 0,534 & 0,224 & $-0,038$ & 3,76 & 3,356 & 553 \\
\hline $\begin{array}{l}\text { Frage 3a Ruheschmerzen (letzte } 24 \text { Stun- } \\
\text { den) }\end{array}$ & 0,834 & 0,239 & $-0,097$ & 3,06 & 2,720 & 553 \\
\hline $\begin{array}{l}\text { Frage } 3 b \text { Belastungsschmerzen (letzte } 24 \\
\text { Stunden) }\end{array}$ & 0,855 & 0,260 & $-0,025$ & 3,44 & 2,848 & 553 \\
\hline $\begin{array}{l}\text { Frage 3c Stärkster Schmerz (letzte } 24 \text { Stun- } \\
\text { den) }\end{array}$ & 0,880 & 0,259 & $-0,058$ & 4,18 & 2,959 & 553 \\
\hline $\begin{array}{l}\text { Frage } 4 \text { a Bewegung durch Schmerzen be- } \\
\text { einträchtigt (letzte } 24 \text { Stunden) }\end{array}$ & 0,591 & 0,285 & 0,083 & 2,54 & 0,499 & 553 \\
\hline $\begin{array}{l}\text { Frage } 4 b \text { Husten oder tiefes Luftholen } \\
\text { durch Schmerzen beeinträchtigt (letzte } 24 \\
\text { Stunden) }\end{array}$ & 0,242 & 0,401 & 0,060 & 2,39 & 0,791 & 553 \\
\hline $\begin{array}{l}\text { Frage 4c Schlafen durch Schmerzen beein- } \\
\text { trächtigt (letzte } 24 \text { Stunden) }\end{array}$ & 0,573 & 0,184 & 0,019 & 2,33 & 0,472 & 553 \\
\hline $\begin{array}{l}\text { Frage } 4 d \text { Stimmung durch Schmerzen be- } \\
\text { einträchtigt (letzte } 24 \text { Stunden) }\end{array}$ & 0,513 & 0,238 & $-0,199$ & 2,36 & 0,480 & 553 \\
\hline $\begin{array}{l}\text { Frage 5a Sehr müde gewesen (letzte } 24 \\
\text { Stunden) }\end{array}$ & 0,374 & 0,494 & 0,158 & 2,58 & 0,495 & 553 \\
\hline $\begin{array}{l}\text { Frage } 5 b \text { Unter Übelkeit gelitten (letzte } 24 \\
\text { Stunden) }\end{array}$ & 0,188 & 0,710 & $-0,029$ & 2,16 & 0,371 & 553 \\
\hline $\begin{array}{l}\text { Frage } 5 c \text { Unter Schwindel gelitten (letzte } \\
24 \text { Stunden) }\end{array}$ & 0,228 & 0,706 & $-0,151$ & 2,29 & 0,456 & 553 \\
\hline $\begin{array}{l}\text { Frage } 5 d \text { Unter Obstipation gelitten (letzte } \\
24 \text { Stunden) }\end{array}$ & 0,094 & 0,467 & $-0,064$ & 2,19 & 0,395 & 553 \\
\hline $\begin{array}{l}\text { Frage } 6 \text { Information über Möglichkeiten } \\
\text { der Schmerztherapie }\end{array}$ & 0,119 & 0,097 & 0,719 & 2,46 & 0,499 & 553 \\
\hline $\begin{array}{l}\text { Frage } 7 \text { Bestand der Wunsch nach mehr } \\
\text { Mitteln gegen Schmerzen }\end{array}$ & $-0,386$ & $-0,173$ & 0,274 & 2,82 & 0,385 & 553 \\
\hline $\begin{array}{l}\text { Frage } 8 \text { Zufriedenheit mit dem Ergebnis } \\
\text { der erhaltenen Schmerztherapie }\end{array}$ & $-0,186$ & $-0,186$ & 0,720 & 7,07 & 2,920 & 553 \\
\hline
\end{tabular}


Tabelle 12: Deskriptive Statistik und Faktorenanalyse nach Entfernung der Fragen 6 bis 8

\begin{tabular}{|c|c|c|c|c|c|}
\hline & \multicolumn{2}{|c|}{ Faktoren } & \multicolumn{3}{|c|}{ Deskriptive Statistik } \\
\hline & 1 & 2 & Mittelwert & Standardabweichung & $\mathrm{n}$ \\
\hline $\begin{array}{l}\text { Frage } 2 b \text { Schmerzstärke bei Kranken- } \\
\text { hausaufnahme }\end{array}$ & 0,742 & 0,253 & 3,18 & 3,293 & 911 \\
\hline $\begin{array}{l}\text { Frage 2c Stärkster Schmerz seit der Kran- } \\
\text { kenhausaufnahme }\end{array}$ & 0,656 & 0,283 & 2,75 & 3,227 & 911 \\
\hline $\begin{array}{l}\text { Frage 3a Ruheschmerzen (letzte } 24 \text { Stun- } \\
\text { den) }\end{array}$ & 0,859 & 0,272 & 2,17 & 2,638 & 911 \\
\hline $\begin{array}{l}\text { Frage } 3 b \text { Belastungsschmerzen (letzte } 24 \\
\text { Stunden) }\end{array}$ & 0,886 & 0,285 & 2,49 & 2,807 & 911 \\
\hline $\begin{array}{l}\text { Frage 3c Stärkster Schmerz (letzte } 24 \\
\text { Stunden) }\end{array}$ & 0,910 & 0,296 & 3,06 & 3,078 & 911 \\
\hline $\begin{array}{l}\text { Frage 4a Bewegung durch Schmerzen } \\
\text { beeinträchtigt (letzte } 24 \text { Stunden) }\end{array}$ & 0,700 & 0,249 & 2,40 & 0,489 & 911 \\
\hline $\begin{array}{l}\text { Frage } 4 \mathrm{~b} \text { Husten oder tiefes Luftholen } \\
\text { durch Schmerzen beeinträchtigt (letzte } \\
24 \text { Stunden) }\end{array}$ & 0,356 & 0,313 & 2,25 & 0,662 & 911 \\
\hline $\begin{array}{l}\text { Frage 4c Schlafen durch Schmerzen be- } \\
\text { einträchtigt (letzte } 24 \text { Stunden) }\end{array}$ & 0,626 & 0,162 & 2,23 & 0,424 & 911 \\
\hline $\begin{array}{l}\text { Frage 4d Stimmung durch Schmerzen } \\
\text { beeinträchtigt (letzte } 24 \text { Stunden) }\end{array}$ & 0,586 & 0,274 & 2,26 & 0,438 & 911 \\
\hline $\begin{array}{l}\text { Frage 5a Sehr müde gewesen (letzte } 24 \\
\text { Stunden) }\end{array}$ & 0,359 & 0,606 & 2,50 & 0,500 & 911 \\
\hline $\begin{array}{l}\text { Frage 5b Unter Übelkeit gelitten (letzte } \\
24 \text { Stunden) }\end{array}$ & 0,188 & 0,711 & 2,14 & 0,344 & 911 \\
\hline $\begin{array}{l}\text { Frage } 5 c \text { Unter Schwindel gelitten (letzte } \\
24 \text { Stunden) }\end{array}$ & 0,219 & 0,710 & 2,26 & 0,440 & 911 \\
\hline $\begin{array}{l}\text { Frage } 5 d \text { Unter Obstipation gelitten } \\
\text { (letzte } 24 \text { Stunden) }\end{array}$ & 0,155 & 0,434 & 2,15 & 0,361 & 911 \\
\hline
\end{tabular}




\subsection{Differentielle Validität von QUIKS}

Die differentielle Validität wurde mit dem Mann-Whitney-U-Test überprüft (Tabelle 13). Aufgrund der starken Heterogenität innerhalb der Hauptdiagnoseverteilung, wurden alle Patienten mit einem Schlaganfall und einer TIA zu einer Gruppe zusammengefasst ( $\mathrm{n}=$ 114) und dann mit allen zur zweiten Gruppe zusammengefassten malignen Erkrankungen $(n=82)$ verglichen. So konnten zwei nahezu ähnlich große Diagnosegruppen erstellt werden. Verglichen wurden die zwei Subskalen aus der Faktorenanalyse zur „Schmerzintensität und funktionellen Beeinträchtigung" und den „therapiebedingten Auswirkungen“. Die jeweiligen Schmerzintensitäten zeigten bei den malignen Neoplasien eine deutlichere, aber nicht signifikant höhere Merkmalsausprägung als bei den Schlaganfällen. Die Intensität der stärksten Behandlungsschmerzen seit der Krankenhausaufnahme war jedoch hingegen bei malignen Neoplasien signifikant höher (Asymptotische Signifikanz, p = 0,02). Die Angaben zu den „funktionellen Beeinträchtigungen“ waren nahezu gleich stark ausgeprägt. Die Merkmalsausprägung „Übelkeit“ zeigte innerhalb der malignen Neoplasien einen sehr signifikanten Unterschied zum Schlaganfall auf (Asymptotische Signifikanz, $p=0,005)$.

Eine deutliche, aber nicht signifikant unterschiedliche Rangverteilung wurde zusätzlich noch im Zusammenhang mit „Schwindel“ und der „Beeinträchtigung der Stimmung“ erkennbar. So war die Ausprägung des Schwindels gehäufter bei Schlaganfallpatienten und die Beeinträchtigung der Stimmung zeigte sich häufiger bei malignen Neoplasien.

\subsection{Ergebnisse der COSMIN-Checkliste}

Es wurden zur Überprüfung die Boxen A, D, E und J mit Hilfe der 4-Punkte-Skala (Abbildung 20 und im Anhang 6.4) herangezogen. Das jeweils schlechteste Ergebnis war ausschlaggebend für die Gesamtbewertung einer Box. Im Konsens mit der Projektleitung wurden die Boxen A und E („Internal consistency“ und "Structural validity“) mit „exzellent“ eingestuft, die BoxD („Content validity“) könnte auch im schlechtesten Fall als „gut“ bewertet werden. Die Box J („Interpretability“) wurde insgesamt 8-mal mit „ja“ und nur einmal mit „nein“ bewertet. 
Tabelle 13: Ergebnis des Mann-Whitney-U-Tests

\begin{tabular}{|c|c|c|c|c|}
\hline & Hauptdiagnose & $\mathrm{n}$ & Mittlerer Rang & p-Werte \\
\hline \multirow{3}{*}{$\begin{array}{l}\text { Frage } 2 b \text { Schmerzstärke bei Kran- } \\
\text { kenhausaufnahme }\end{array}$} & Maligne Neoplasie Sonstiges & 81 & 105,06 & \multirow{3}{*}{0,077} \\
\hline & Schlaganfall & 113 & 92,08 & \\
\hline & Gesamt & 194 & & \\
\hline \multirow{3}{*}{$\begin{array}{l}\text { Frage 2c Stärkster Schmerz seit der } \\
\text { Krankenhausaufnahme }\end{array}$} & Maligne Neoplasie Sonstiges & 80 & 107,29 & \multirow[t]{3}{*}{0,020} \\
\hline & Schlaganfall & 113 & 89,72 & \\
\hline & Gesamt & 193 & & \\
\hline \multirow{3}{*}{$\begin{array}{l}\text { Frage 3a Ruheschmerzen (letzte } 24 \\
\text { Stunden) }\end{array}$} & Maligne Neoplasie Sonstiges & 82 & 103,85 & \multirow[t]{3}{*}{0,212} \\
\hline & Schlaganfall & 114 & 94,65 & \\
\hline & Gesamt & 196 & & \\
\hline \multirow{3}{*}{$\begin{array}{l}\text { Frage } 3 \mathrm{~b} \text { Belastungsschmerzen } \\
\text { (letzte } 24 \text { Stunden) }\end{array}$} & Maligne Neoplasie Sonstiges & 81 & 100,52 & \multirow[t]{3}{*}{0,556} \\
\hline & Schlaganfall & 114 & 96,21 & \\
\hline & Gesamt & 195 & & \\
\hline \multirow{3}{*}{$\begin{array}{l}\text { Frage 3c Stärkster Schmerz (letzte } \\
24 \text { Stunden) }\end{array}$} & Maligne Neoplasie Sonstiges & 80 & 100,62 & \multirow[t]{3}{*}{0,417} \\
\hline & Schlaganfall & 113 & 94,44 & \\
\hline & Gesamt & 193 & & \\
\hline \multirow{3}{*}{$\begin{array}{l}\text { Frage } 4 a \text { Bewegung durch Schmer- } \\
\text { zen beeinträchtigt (letzte } 24 \text { Stun- } \\
\text { den) }\end{array}$} & Maligne Neoplasie Sonstiges & 80 & 99,68 & \multirow[t]{3}{*}{0,548} \\
\hline & Schlaganfall & 114 & 95,97 & \\
\hline & Gesamt & 194 & & \\
\hline \multirow{3}{*}{$\begin{array}{l}\text { Frage } 4 b \text { Husten oder tiefes Luftho- } \\
\text { len durch Schmerzen beeinträchtigt } \\
\text { (letzte } 24 \text { Stunden) }\end{array}$} & Maligne Neoplasie Sonstiges & 80 & 98,65 & \multirow[t]{3}{*}{0,470} \\
\hline & Schlaganfall & 113 & 95,83 & \\
\hline & Gesamt & 193 & & \\
\hline \multirow{3}{*}{$\begin{array}{l}\text { Frage 4c Schlafen durch Schmerzen } \\
\text { beeinträchtigt (letzte } 24 \text { Stunden) }\end{array}$} & Maligne Neoplasie Sonstiges & 79 & 98,77 & \multirow[t]{3}{*}{0,596} \\
\hline & Schlaganfall & 114 & 95,78 & \\
\hline & Gesamt & 193 & & \\
\hline \multirow{3}{*}{$\begin{array}{l}\text { Frage } 4 d \text { Stimmung durch Schmer- } \\
\text { zen beeinträchtigt (letzte } 24 \text { Stun- } \\
\text { den) }\end{array}$} & Maligne Neoplasie Sonstiges & 80 & 100,75 & \multirow[t]{3}{*}{0,344} \\
\hline & Schlaganfall & 114 & 95,22 & \\
\hline & Gesamt & 194 & & \\
\hline \multirow{3}{*}{$\begin{array}{l}\text { Frage } 5 \text { a Sehr müde gewesen (letzte } \\
24 \text { Stunden) }\end{array}$} & Maligne Neoplasie Sonstiges & 81 & 97,65 & \multirow[t]{3}{*}{0,932} \\
\hline & Schlaganfall & 114 & 98,25 & \\
\hline & Gesamt & 195 & & \\
\hline \multirow{3}{*}{$\begin{array}{l}\text { Frage } 5 b \text { Unter Übelkeit gelitten } \\
\text { (letzte } 24 \text { Stunden) }\end{array}$} & Maligne Neoplasie Sonstiges & 81 & 106,57 & \multirow[t]{3}{*}{0,005} \\
\hline & Schlaganfall & 114 & 91,91 & \\
\hline & Gesamt & 195 & & \\
\hline \multirow{3}{*}{$\begin{array}{l}\text { Frage } 5 c \text { Unter Schwindel gelitten } \\
\text { (letzte } 24 \text { Stunden) }\end{array}$} & Maligne Neoplasie Sonstiges & 82 & 93,73 & \multirow[t]{3}{*}{0,164} \\
\hline & Schlaganfall & 114 & 101,93 & \\
\hline & Gesamt & 196 & & \\
\hline \multirow{3}{*}{$\begin{array}{l}\text { Frage } 5 d \text { Unter Obstipation gelitten } \\
\text { (letzte } 24 \text { Stunden) }\end{array}$} & Maligne Neoplasie Sonstiges & 82 & 99,02 & \multirow[t]{3}{*}{0,748} \\
\hline & Schlaganfall & 113 & 97,26 & \\
\hline & Gesamt & 195 & & \\
\hline
\end{tabular}




\subsection{Chronische Schmerzen als Beispiel einer inhaltlichen Auswertung}

Als ein weiteres Beispiel für die inhaltliche Validität von QUIKS wurde das Kriterium vorbestehender chronischer Schmerzen herangezogen.

Die bestehenden chronischen Schmerzen wurden mit Frage 1 erfasst und hatten auch erkennbaren Einfluss auf die Ergebnisse der darauffolgenden Fragen. Hier zeigte sich auch im Gruppenvergleich (Kapitel 3.2.1), dass Frauen im Vergleich zu Männern deutlich häufiger einen chronischen Schmerz angaben. Patienten mit chronischen Schmerzen gaben eine durchweg höhere Schmerzintensität und häufiger funktionelle Beeinträchtigungen im Alltag an, als nicht chronische Schmerzpatienten. Diese Erkenntnisse decken sich grundsätzlich auch mit denen von vielen anderen Voruntersuchungen. Bei den Angaben zu den Schmerzintensitäten lag der Anteil der Patienten ohne chronische Schmerzen mit einer subjektiven Schmerzfreiheit bei jeweils etwa 50\% pro Frage, in jeweils weniger als $30 \%$ gaben sie eine funktionelle Beeinträchtigung im Alltag an. Insgesamt wurden von den Patienten ohne chronische Schmerzen geringere Ruhe- und Belastungsschmerzintensitäten angegeben. Trotz der insgesamt höheren Schmerzintensitäten gab auch bei chronischen Schmerzpatienten ein relevanter Teil eine subjektive Schmerzfreiheit an ( $A \boldsymbol{b}$ -

\section{bildung 19).}

Gleichzeitig hatte der chronische Schmerz auch Einfluss auf die jeweiligen Schmerzentitäten, denn chronische Schmerzpatienten gaben vermehrt an, während des Aufenthaltes am schlimmsten unter Rücken- (13\%) oder Gelenkschmerzen (12\%) zu leiden. Im Vergleich dazu waren die führenden Schmerzentitäten nicht chronischer Schmerzpatienten Kopf- (5\%), Rücken- und Bauchschmerzen (je 4\%) und Schmerzen bei Untersuchungen (3\%). Patienten mit chronischen Schmerzen beantworteten die Tabelle der möglichen Schmerzentitäten während des Krankenhausaufenthaltes in Frage 2a insgesamt häufiger und zudem auch häufiger korrekt (Tabelle 14). Ein Rückschluss auf die für die chronischen Schmerzen ursächliche Schmerzentität lässt sich aus dem QUIKS Fragebogen nicht ableiten.

Ein weiterer Aspekt zeigte sich bei den Angaben zur Patientenzufriedenheit. Die Patienten ohne chronische Schmerzen $(n=199)$ gaben genau wie Patienten mit chronischen Schmerzen ( $n=315$ ) auf der NRS nahezu übereinstimmend zufriedene Werte an (Median 8; 5 - 10). Im Zusammenhang mit der Zufriedenheit über eine Schmerztherapie 
wurde zusätzlich ausgewertet, inwiefern sich die Häufigkeiten bei der Beantwortung zwischen Patienten mit chronischen und nicht chronischen Schmerzen unterscheiden (Tabelle 14). Hier lässt sich erkennen, dass Patienten mit chronischen Schmerzen die jeweiligen Fragen häufiger beantwortet haben.
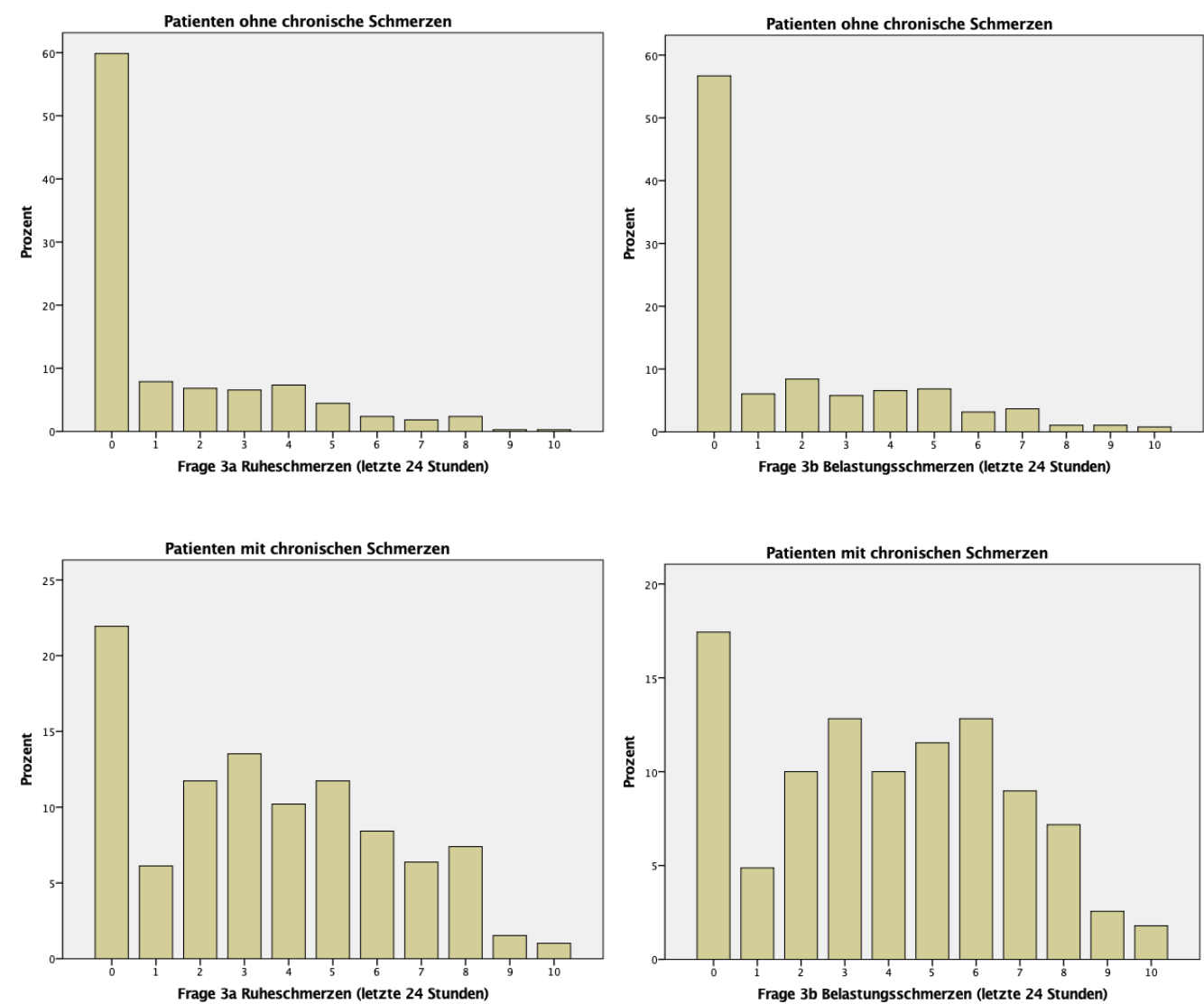

Abbildung 19: Grafische Darstellung der Schmerzintensitäten der Ruhe- und Belastungsschmerzen zwischen Patienten mit und ohne chronische Schmerzen

Tabelle 14: Übersicht über die Beantwortung von möglichen Schmerzindikatorfragen (2a, $2 b, 3 c$ und 8) durch Patienten mit chronischen und nicht chronischen Schmerzen (\% in Bezug auf Anzahl der Patienten mit und ohne chronische Schmerzen)

\begin{tabular}{|c|c|c|c|c|}
\hline \multirow{2}{*}{$\begin{array}{l}\text { Gesamt Frage } 1 a \\
\text { Tabelle } 2 a\end{array}$} & \multicolumn{2}{|c|}{$\begin{array}{c}\text { Chronische Schmerzen } \\
\qquad n=393(51 \%)\end{array}$} & \multicolumn{2}{|c|}{$\begin{array}{l}\text { Nicht chronische Schmerzen } \\
\qquad n=383(49 \%)\end{array}$} \\
\hline & 386 & $98 \%$ & 269 & $70 \%$ \\
\hline Tabelle 2 a richtig beantwortet & 232 & $59 \%$ & 135 & $35 \%$ \\
\hline Aufnahmeschmerzen & 390 & $99 \%$ & 376 & $98 \%$ \\
\hline Maximalschmerzen & 390 & $99 \%$ & 379 & $99 \%$ \\
\hline Patientenzufriedenheit & 315 & $80 \%$ & 199 & $52 \%$ \\
\hline
\end{tabular}




\section{Diskussion}

Im Jahr 2010 beschrieben Maier et al. (2010) nach einer umfangreichen Studie im Rahmen des Projekts "Schmerzfreies Krankenhaus" signifikante Defizite in der schmerztherapeutischen Versorgung nicht operativer Patienten im Krankenhaus. Auch wenn es in der postoperativen Schmerztherapie ebenfalls noch immer Handlungsbedarf gibt, existieren zur Versorgung der operativen Patienten schon seit Jahren umfangreiche Konzepte und Qualitätssicherungsinstrumente wie z. B. QUIPS oder PAIN OUT, die besonderes Augenmerk auf die subjektive Bewertung der Qualität der Schmerztherapie durch den Patienten legen (Meissner et al. 2008; Rothaug et al. 2013; Rothaug 2013; Erlenwein 2016; Meißner 2016). Zudem erfassen sie neben der subjektiven Qualitätsbeurteilung nicht nur die reinen Schmerzintensitäten, sondern auch die schmerzbedingten funktionellen und therapiebedingten Beeinträchtigungen nach der Operation. Entwickelt wurden sie als Benchmarking-Projekte, die durch die stetige Analyse der eigenen Ergebnisqualität und sofortigen Rückmeldung an den Anwender eine nachweisliche Qualitätsverbesserung bewirkt haben (Meissner et al. 2008; Rothaug et al. 2013). Da solche wirkungsvollen schmerztherapeutischen Konzepte bislang überwiegend auf Seiten der postoperativen Versorgung vorhanden sind und dort täglich im klinischen Alltag eingesetzt werden, war es nun das Ziel dieser Promotionsarbeit die großflächigere Testung eines Qualitätssicherungsinstruments für die Schmerztherapie bei konservativ behandelten Patienten umzusetzen. Das dafür entwickelte QUIKS-Modul soll nach der erfolgreichen klinischen Einführung langfristig gesehen mit dem QUIPS-Projekt zusammengeführt werden. Entsprechend orientierte sich QUIKS inhaltlich, soweit angemessen an QUIPS und wurde für den konservativen Bereich durch zusätzliche Fragestellungen und Inhalte ergänzt. Auf dieser Grundlage, sowie den Publikationen von Meißner et al. (2008) und Rothaug et al. (2013) zur Validierung von QUIPS wurde der QUIKS-Fragebogen erstellt und auf praktische Anwendbarkeit und Konsistenz sowie Validität getestet.

Nach der Auswertung der Ergebnisse zeigte sich, dass es mit QUIKS durchaus möglich ist eine für nicht operativ behandelte Patienten umfangreiche und sinnvolle Erhebung zur Qualität der Schmerztherapie durchführen zu können. Es war möglich, Informationen aus vielen schmerztherapeutisch relevanten Bereichen zu erhalten, wie beispielsweise vorbestehende chronische Schmerzen in der Klinik, jeweilige Schmerzintensitäten und Be- 
einträchtigungen im stationären Alltag. Zudem konnten Medikationen und deren Anpassung sowie Diagnosen unabhängig vom ICD als Grundlage für ein Benchmarking erfasst werden.

Inhaltlich zeigte sich in den Auswertungen auch, dass QUIKS relevante Auswirkungen auf das individuelle Schmerzerleben zwischen verschiedenen Patientengruppen erfassen konnte. Die psychometrischen Ergebnisse waren mit denen von QUIPS durchaus vergleichbar, wenn auch hier eine größere Heterogenität der klinischen Situation deutlich wurde.

\subsection{Diagnosen als Grundlage für Benchmarking}

Am Befragungstag lagen zwar bereits 94\% der Diagnosen konkret formuliert vor und konnten mit der erstellten Wortdiagnoseliste erfasst werden, es stellten sich aber auch besondere Herausforderungen dar, wie z. B. einen für konservativ behandelte Patienten sinnvoll anwendbaren Benchmarking-Faktor in Bezug auf zu vergleichende Patienten, bzw. Diagnosegruppen festlegen zu können. In den postoperativen Projekten ist der Benchmarking-Faktor die klare Zuordnung der jeweiligen Operationen mittels OPS und den dazugehörigen Fachabteilungen. Ursprünglich wurde als potentielle Vergleichsmöglichkeit die mittels ICD-10 kodierte Hauptdiagnose angestrebt. Bereits in der ersten Testphase zeigte sich jedoch, dass eine bereits kodierte Hauptdiagnose am Befragungstag nicht überall dokumentiert vorlag. Somit wurde die Variante mit der ICD-10-Kodierung verworfen und eine ankreuzbare Wortdiagnoseliste erstellt, in die der Patient eingruppiert werden konnte. Nach der Auswertung der bisherigen Ergebnisse zeigte sich, dass die Heterogenität innerhalb der Hauptdiagnosen sehr vielfältig war und Patienten mit der aktuellen Version nur eingeschränkt umfassend erhoben werden konnten - als wichtige Voraussetzung für ein zukünftiges Benchmarking. Andererseits stellt sich die Frage welches Niveau der diagnostischen Differenzierung zur Vergleichbarkeit im Benchmarking sinnvoll und notwendig ist. Allein 26\% der konkreten Hauptdiagnosen wurden namentlich unter „sonstige Hauptdiagnose“ erfasst, da sie sich noch nicht in der Liste befanden. Hieraus ist ableitbar, dass in der aktuellen Tabelle noch viele auch häufige Diagnosen fehlen und von einer notwendigen Anpassung mit Vergrößerung des QUIKS-Datensatzes auszugehen ist. Letztlich blieb nur bei 6,2\% der Patienten die Hauptdiagnose nicht erhebbar. So könnten Grenzwerte für Häufigkeiten überlegt werden, um eine Diagnose in die Liste 
fest aufzunehmen oder weiterhin nicht spezifisch unter "Sonstiges" zu erfassen. Ein Vergleich zwischen den verschiedenen Diagnoseobergruppen wäre ebenfalls denkbar und stellt sicherlich einen ersten Schritt dar, könnte möglicherweise aber auch zu oberflächlich gefasst sein, um damit eine relevante Vergleichbarkeit in einem Benchmarking zu erzielen.

Aus der großen Heterogenität lässt sich zudem auch für das zukünftige Benchmarking überlegen, auf welcher Ebene die konservativ behandelten Patienten mit QUIKS verglichen werden sollen und wie die Tabelle daraufhin angepasst werden müsste. Denkbar wären hier auch Ansätze, nicht alle konservativ behandelten Patienten, sondern nur Patienten mit tatsächlicher Schmerzangabe einzuschließen, immerhin 63\% der Befragten.

Wie aber bereits im Ergebnisteil angesprochen, machten viele Patienten inkonsistente Angaben. Kritisch bleibt, wie dabei eine „positive Schmerzanagabe“ aus QUIKS abgeleitet bzw. definiert wird. Die in der Auswertung genutzte Frage 3c (maximaler Schmerz in den letzten $24 \mathrm{~h}>1$ ) als Kennwert für stattgehabte Schmerzen war hier womöglich zu sensitiv und nicht für alle Patienten klinisch relevant. Schließlich war es den Patienten auch in anderen Fragen möglich, empfundene Schmerzen anzugeben, wie über das Bestehen von chronischen Schmerzen, in der Tabelle zu empfundenen Schmerzentitäten (mit Aufnahme- und Behandlungsschmerzen) und über die Schmerzintensitäten in Frage 3. Für den postoperativen Bereich wird zwar eine Schmerzbewertung von NRS >3 als Trigger für eine mögliche Intervention bewertet, aber auch kritisch diskutiert (Gerbershagen 2011). Auf den hier untersuchten Kontext der konservativen Patienten ist die postoperative Situation nicht übertragbar, eine Bewertung der Schmerzscores in diesem Kontext ist folglich auch nicht sinnvoll. Daten zur Epidemiologie chronischer Schmerzen weisen auch daraufhin, dass zwar 27,3\% der Bevölkerung Schmerzen von mindestens drei Monaten Dauer angeben, diese aber nur bei einem deutlich geringeren Anteil mit funktionellen und psychosozialen Beeinträchtigungen einher gehen (Häuser et al. 2014). Hieraus lassen sich viele potenzielle Störquellen für eine konsistente Beantwortung bei interessanten und klinisch wichtigen Ansätzen für eine Auswertung ableiten, die aber die Möglichkeiten des vorliegenden Datensatzes überschreiten, wie beispielsweise ob Schmerzen überhaupt als beeinträchtigend empfunden wurden. 
Um den oder die Benchmarking-Faktor(en) hier noch einmal zu überprüfen und optimieren zu können, sollte diese Problematik mit den noch offenen Fragen in zukünftigen Projekten an einer größeren Klinikzahl weiter diskutiert werden.

\subsection{Spezielle Analyse im Literaturvergleich}

Die von Maier et al. (2010) aufgezeigten Defizite im Bereich der Schmerztherapie nicht operativer Patienten konnten mit QUIKS an der Universitätsmedizin Göttingen, dem Universitätsklinikum Jena und dem Krankenhaus Martha-Maria in Halle nicht in vergleichbarer Relevanz und Ausprägung aufgezeigt werden. Auch die Teilnehmerquote der Patienten an der Studie zeigte mit 74\% höhere Werte im Vergleich zu Maier et al. (61,4\%).

Trotz der viel geringeren Anzahl teilnehmender Krankenhäuser in QUIKS ( $n=3$ vs. 25) konnte eine annähernd gleich große Stichprobe nicht operativ behandelter Patienten erhoben werden ( $n=973$ vs. 999). Weit mehr als die Hälfte aller Patienten gaben auf nahezu allen NRS Schmerzintensitäten unterhalb der oft als Interventionstrigger beschriebenen NRS-Werte von 4 an. Fast die Hälfte der Patienten waren auch weitgehend subjektiv schmerzfrei (52\% Männer, 41\% Frauen), bei der Veröffentlichung von 2010 waren es nur 16,7\%. Ebenfalls gaben die meisten Patienten mit Schmerzen an, keinen „Wunsch nach mehr Schmerzmitteln“ verspürt zu haben (81\%) und waren im Median mit 8 auf der NRS der subjektiven Zufriedenheit (0 bis 10) zufrieden mit der aktuellen Versorgung. Insgesamt gaben 33\% dieser Patienten NRS-Werte $<6$ als Hinweis auf eine geringe Zufriedenheit an. Auch unter Berücksichtigung der Erhebung der Daten an nur drei teilnehmenden Kliniken ist die Zufriedenheit 2016 erhöht im Vergleich zu Maier et al. (2010), in der noch mehr als 50\% unzufrieden waren.

Es konnte daher gezeigt werden, dass die Patienten zumindest in diesen drei Kliniken nicht in dem Maße schmerztherapeutisch unterversorgt waren, wie es noch bei Maier et al. (2010) der Fall war. Möglicherweise haben aber auch hier bereits in den zehn Jahren zwischen den beiden Studien spürbare Verbesserungen der klinikinternen Methoden, Prozesse und Strukturen stattgefunden, oder Patienten kommen bereits besser vorbehandelt in die Klinik, da in der QUIKS-Erhebung nur ein Teil eine relevante Modifikation der medikamentösen Therapie erhielt. Auch bei der differenzierten Auswertung von Patienten mit einem vorbestehenden chronischen Schmerz waren diese Zufriedenheits- 
werte hoch. Hier könnte aber auch überlegt werden, ob sich neben den bereits genannten möglichen Verbesserungen in der stationären Versorgung, einfach nur die Erwartungen von chronischen Schmerzpatienten widerspiegeln, die darüber froh sind, wenn es in der Klinik so weiterläuft wie zuvor oder sie differenzieren die Gründe für die Aufnahme von ihrem chronischen Schmerz.

Gleichzeitig muss hier jedoch darauf hingewiesen werden, dass die vorliegenden Ergebnisse keinen repräsentativen Ausschnitt widerspiegeln und deshalb in zukünftigen Studien repliziert und hinterfragt werden sollten.

Um die externe Validität des QUIKS-Erhebungsfragebogens zu überprüfen, wurden zwei verschiedene Gruppen von Diagnosen miteinander verglichen. Die zu zwei Gruppen zusammengefassten Diagnosen Schlaganfall und maligne Neoplasien zeigten in der Auswertung die Unterschiede, die auch aus klinischer Sicht zu erwarten waren. So wurden beispielsweise höhere Behandlungsschmerzen und vermehrt Übelkeit von den Patienten mit malignen Neoplasien angegeben. Patienten mit einem Schlaganfall litten häufiger unter "Schwindel“. Die vorliegenden Ergebnisse zeigen somit, dass trotz der Heterogenität eine grundsätzliche und klinisch sinnvolle Anwendbarkeit des QUIKS-Moduls gegeben ist. Diese erste erfolgreiche Messung der externen Validität von QUIKS ist daher auch schon mit der Validität von QUIPS vergleichbar (Rothaug et al. 2012).

Die allgemeine statistische Auswertung der postoperativen Erhebungsinstrumente zeigte in den vorangegangenen Publikationen von Meißner et al. (2008), Maier et al. (2010) und Rothaug et al. (2013) weniger Schwierigkeiten in der praktischen Umsetzung und korrekten Verwendung durch die Patienten, als dies bei QUIKS der Fall war. Es wurde deutlich, dass sich die sehr heterogene Patientenklientel (insbesondere auch in Bezug auf Schmerz) als schwieriger zu erfassen darstellt. Im Fall von QUIKS wurde dies vor allem mit den Angaben zur Patientenzufriedenheit deutlich, da hier genau die Patienten häufiger keine Angaben machten, die im Vorfeld subjektiv keine Schmerzen empfunden oder in ihren Augen keine Therapie erhalten hatten. Interessanterweise wurden hier aber auch die bereits erwähnten widersprüchlichen oder doppeldeutigen Angaben gemacht, die auf ein fehlerhaftes Verständnis der Fragen hindeuten. Ein sehr wahrscheinlicher Unterschied zu postoperativen Patienten könnte hier sein, dass in der Regel sowohl die Patienten als auch das therapeutische Team postoperativ zumindest von einem individuellen 
Minimalschmerz und dem daraus resultierenden möglichen Bedarf einer Therapie ausgehen.

Bei besserer Charakterisierung und Differenzierung der eigentlichen Schmerzproblematik und erfolgreicher Erprobung/Überarbeitung der Benchmarking-Faktor werden, kann QUIKS ein sinnvolles und sehr hilfreiches Qualitätssicherungsinstrument im Rahmen der konservativen Schmerztherapie darstellen.

\subsubsection{Limitationen}

Viele Limitationen überschneiden sich auch aufgrund der jeweiligen Projektnähe sehr mit denen von QUIPS und PAIN OUT. So kann auch QUIKS mit bisher nur drei teilnehmenden Krankenhäusern keinen bundesweit repräsentativen Zustand abbilden, da hier bereits im Vorfeld möglicherweise eine Selektion durch interessiertere Kliniken stattgefunden haben könnte. Auch der angesprochene Vergleich mit den vorangegangenen Studien von Maier et al. (2010), Meißner et al. (2008) und Rothaug et al. (2013) sollte daher durchaus zurückhaltend und nur mit Vorsicht durchgeführt werden. Dazu kommt, dass nicht in allen teilnehmenden Kliniken ein ähnlich breites Spektrum an Patienten und Fachdisziplinen erfasst werden konnten, zum Teil sind auch erst während der Befragungen Fachdisziplinen dem Projekt beigetreten. In Jena wurden beispielsweise nur neurologische Patienten befragt, in Göttingen dafür nahezu das gesamte Spektrum der konservativen Medizin, wenn auch in unterschiedlichem Ausmaß.

Zudem müssen bei der Auswertung von QUIKS alle Werte selbstverständlich unter Berücksichtigung der nicht zwangsläufig gleich großen und gut selektierten Vergleichsgruppen (wie beispielweise bei der Auswertung Schlaganfall und onkologische Patienten mit $n=114$ vs. 82) betrachtet werden und sollten in zukünftigen Projekten repliziert werden. Auch das Befragungsmuster mit einer einmaligen Befragung am dritten Aufnahmetag konnte viele Patienten nicht miteinschließen, da diese bereits wieder entlassen worden waren. Gleichzeitig bildet die Befragung auch nur eine Momentaufnahme ab, die mögliche schmerzhafte Interventionen in der Folge nicht erfassen kann. Durch den komplexeren Fragentypus setzt QUIKS auch voraus, dass die Patienten kognitiv in der Lage sein müssen den Fragebogen korrekt zu verstehen. Daraus resultiert, dass z. B. demenzielle oder anders kognitiv eingeschränkte Patienten mit Schmerzen möglicherweise überhaupt nicht, fehlerhaft oder unvollständig mit QUIKS erfasst werden können, obwohl sie in einigen konservativen Fachdisziplinen zum täglichen Patientenkollektiv gehören. 
Ein möglicherweise für die Auswertung der Ergebnisse limitierender Faktor, der sich speziell auf das Patientenkollektiv von QUIKS bezieht, könnte auch sein, dass die Patienten an zu vielen verschiedenen Stellen innerhalb des Fragebogens angeben können Schmerzen empfunden zu haben. Dies ist der Komplexität der Szenarien geschuldet (z. B. Schmerz als Aufnahmegrund, vorbestehender chronischer Schmerz, Schmerz durch Prozeduren/Maßnahmen) und dem Versuch hier differenzierte Aussagen zu ermöglichen. Durch ein fehlerhaftes Verständnis können hier in der Folge Inkonsistenzen durch die gegebene Vielzahl an Auswahlmöglichkeiten entstehen. Besonders das bereits angesprochene Patientenkollektiv mit eingeschränkter kognitiver Leistungsfähigkeit könnte hier möglicherweise eine problematische Gruppe sein.

Da in QUIKS nicht zwangsläufig eine schmerzhafte Intervention, wie z. B. eine Operation in Bezug auf QUIPS, im Zentrum des Krankenhausaufenthalts steht, kann eine inhaltliche Bewertung der Patientenaussagen schwierig werden. Dies wurde beispielsweise bei der Auswertung der QUIKS Ergebnisse zur Patientenzufriedenheit mit der Schmerztherapie deutlich, da es hier gehäuft zu inkonsistenten Antworten kam und der eigentliche Bezug zur Schmerztherapie nicht deutlich wurde.

Andererseits könnten durch die Ergebnisse zukünftig komplexe Auswertungen unter verschiedensten Gesichtspunkten angegangen werden, beispielsweise wie bewerten Patienten mit bestehenden chronischen Schmerzen die Situation in der Klinik im Vergleich zu Patienten ohne chronische Schmerzen aber mit einem akuten Schmerz und wie zufrieden waren sie dann jeweils mit der Schmerzbehandlung. Dabei müssen jedoch die genannten Aspekte kritisch gewürdigt werden.

\subsubsection{Bewertung mit Hilfe der COSMIN-Checkliste}

Die COSMIN-Checkliste wurde entwickelt, um eine standardisierte Bewertung der Qualität eines medizinischen Messinstruments und ihrer statistischen Analyse durchführen zu können. Hierbei wird besonderes Augenmerk auf die spezifischen Eigenschaften der Messmethoden für patientenbezogene Ergebnisparameter, z. B. in Patientenfragebögen, gelegt (Mokkink et al. 2006; Mokkink et al. 2010a; Mokkink et al. 2010b; Terwee et al. 2012). Die Checkliste ist so aufgebaut, dass der Anwender die zu bewertende Studie oder Publikation mit Hilfe von einfachen Themenboxen (z. B. zur internen Konsistenz, Reliabilität oder strukturellen Validität) eigenständig überprüfen kann. Da nicht alle Validierungen von Instrumenten sämtliche Aspekte der Checkliste beinhalten, kann der Anwender 
ebenfalls selbstständig festlegen, welche Eigenschaften in der Studie durchgeführt wurden und somit daraufhin zu überprüfen sind. Neben der reinen Checkliste liegen von COSMIN auch eine kommentierte Version zur Bewertung der Validierung eines Instruments mit einer 4-Punkte-Skala für jede Themenbox (exzellent, gut, angemessen, schlecht) und ein umfangreiches Handbuch vor, die dem Anwender eine erstmalige Anwendung erleichtern. Für die Gesamtbeurteilung der Qualität einer Themenbox ist jeweils die schlechteste Bewertung ausschlaggebend.

Im Falle von QUIKS wurden die Boxen A, D, E und J mit Hilfe der 4-Punkte-Skala (Abbildung 20 und im Anhang 6.4.) angewendet und zeigten ein durchaus zufriedenstellendes Ergebnis. Die Themenboxen B und C der COSMIN-Checkliste setzen einen unabhängigen ReTest voraus, weswegen sie bei dieser Bewertung nicht miteingeschlossen wurden. In einer möglichen Folgestudie sollten diese Ergebnisse dann aber bedacht und ggf. mitberücksichtigt werden.

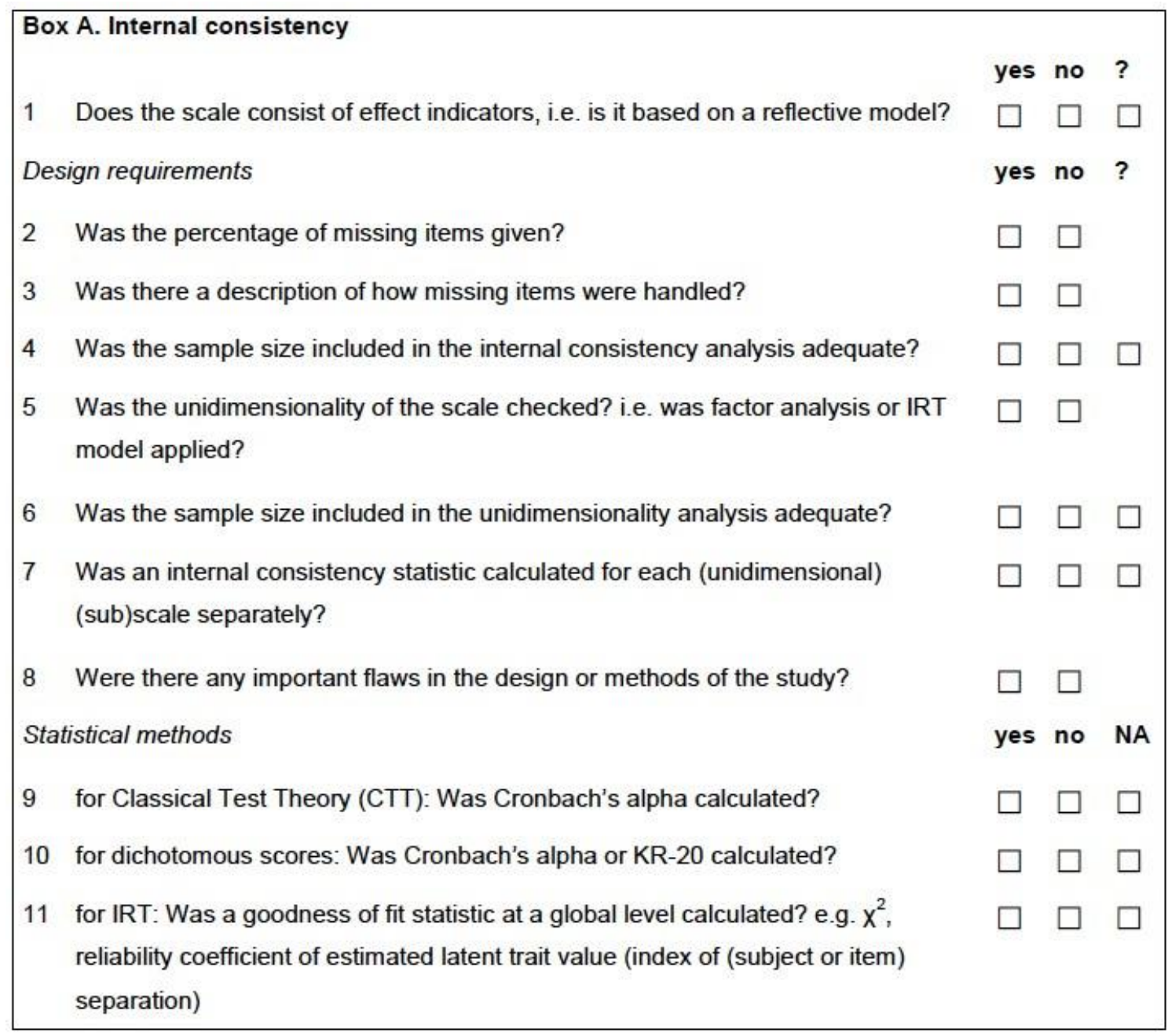

Abbildung 20: Box A hier exemplarisch für alle anderen Boxen der verwendeten COSMINCheckliste (mit freundlicher Genehmigung von VUmc, www.cosmin.nl) 


\subsection{Potenzielle Veränderungen an QUIKS}

Durch den nahezu identischen Aufbau wird die geplante Integrierung des QUIKS-Moduls in das QUIPS-Register vermutlich komplikationslos verlaufen können. Trotz der umfangreichen Testphasen vor Beginn des eigentlichen Projekts stellten sich aber einige Probleme und Limitationen mit dem Ergebnisfragebogen und den Prozessparametern immer wiederkehrend dar. Vor allem Patienten im höheren Lebensalter oder mit einer hohen Komorbidität zeigten sich häufig und schnell überfordert. In der Folge sollen daher einige Vorschläge zur möglichen Veränderung des QUIKS-Instruments diskutiert werden. Grundsätzlich sollte jedoch die inhaltliche Struktur weiterhin so beibehalten werden, um nicht zu sehr vom bewährten QUIPS-Konzept abzuweichen und die Zusammenführung so zu erschweren. Denn die grundsätzliche Anwendbarkeit konnte in dieser Form sowohl in dieser Projektphase als auch mit QUIPS und PAIN OUT über viele Jahre hinweg gezeigt werden.

Wie bereits im Ergebnisteil dieser Arbeit deutlich wurde, zeigten sich auffallende Schwierigkeiten bei der Beantwortung der Frage 1, der Tabelle in Frage 2a und bei der Frage 8 mit den Angaben zur Patientenzufriedenheit. Daher werden in der Folge verschiedene Möglichkeiten zur Anpassung insbesondere der betroffenen Fragen diskutiert.

\subsubsection{Veränderungen an Frage 1}

Die ursprüngliche erste Frage wurde so ausgewählt, dass die Fragen 1b und 1c abhängig von der Beantwortung von Frage 1a waren. Leider zeigte sich, dass die Formulierung der Frage in 15\% der Fälle nicht deutlich genug war, wodurch es hier zu einer fehlerhaften Beantwortung der gesamten Frage kam. In den meisten Fällen wurde zwar bei einer Verneinung die Frage 1b nicht beantwortet, 1c wurde aber wieder angekreuzt. In sehr vielen Fällen wurden aber auch alle drei Fragen unabhängig von 1a beantwortet.

Das zeigt, dass die Gestaltung dieser Frage noch Mängel aufweist, die für die Praxiseinführung verbessert werden könnten.

Eine mögliche einfache Optimierung könnte die optische Anpassung der Folgefragen 1b und 1c sein, indem man diese ein Stück einrückt und so eine Unterordnung zu 1a hervorhebt (Abbildung 21). Gleichzeitig könnten die Formulierungen der beiden Folgefragen noch einmal verdeutlicht werden, um mit diesen Maßnahmen den Leser von einer zu schnellen und falschen Beantwortung abzuhalten. 
1a. Hatten Sie ständige Schmerzen, die drei Monate oder länger andauerten, bevor Sie ins Krankenhaus gekommen sind?

口 Ja a Nein

Wenn 1a=Ja, bitte beantworten: 1b. Wie stark waren diese Schmerzen für Sie meistens?

\begin{tabular}{|l|l|l|l|l|l|l|l|l|l|r|}
\hline $\mathbf{0}$ & $\mathbf{1}$ & $\mathbf{2}$ & $\mathbf{3}$ & $\mathbf{4}$ & $\mathbf{5}$ & $\mathbf{6}$ & $\mathbf{7}$ & $\mathbf{8}$ & $\mathbf{9}$ & $\mathbf{1 0}$ \\
\hline $\begin{array}{l}\text { Kein } \\
\text { Schmerz }\end{array}$ & \multicolumn{10}{|c|}{$\begin{array}{r}\text { Stärkster } \\
\text { vorstelllbarer } \\
\text { Schmerz }\end{array}$} \\
\hline
\end{tabular}

Wenn 1a=Ja, bitte beantworten: 1c. Waren diese Schmerzen Grund für Ihre Krankenhausaufnahme?

a Ja a Nein a Weiß nicht

Abbildung 21: Änderungsvorschlag, Frage 1

Eine weitere Alternative wäre eine webbasierte Fragebogenanwendung mittels Computer oder Tablet. Hier könnte über die Software programmiert werden, dass die Folgefragen nur erscheinen, wenn sie auch sinnvollerweise erscheinen sollen. Computer oder Tablets können dann zur Fehlerrate „in Papier" in Relation gesetzt und abgewogen werden. Andererseits könnte die Handhabung eines Computers besonders bei älteren Patienten erneut zu einer höheren Abschreckung statt zu mehr Sicherheit führen. Eine zu bedenkende Reaktion könnte daraufhin auch sein, dass vermehrt Patienten aus Unsicherheit eine Befragung im Vorfeld ablehnen oder die Interviewrate merklich ansteigen könnte.

Auch wenn mit einer direkten webbasierten Eingabe vor Ort ein folgender Prozessschritt, die Eingabe in die Benchmarking-Datei, entfallen könnte, sollte zunächst die einfache optische Anpassung erprobt und die Fehlerrate erneut geprüft werden. In der Folge wäre die kostenintensive Anschaffung einer Hard- und Software immer noch zu realisieren.

\subsubsection{Veränderungen an der Tabelle}

Eine weitere Schwierigkeit zeigte sich nach der Einführung der Tabelle zu Schmerzentitäten (Frage 2a), mit der es den Patienten möglich war eine konkrete Angabe über empfundene Schmerzlokalisation machen zu können, auch wenn diese nicht mit der eigentlichen Einweisungsdiagnose bzw. Erkrankung zusammenhingen. Bei dieser Frage wurde sehr schnell deutlich, dass die letztlich eingeführte Tabelle in der aktuellen Form von den meisten Patienten nicht wie gewünscht angewendet wurde. Wie schon zuvor wirkten besonders ältere Patienten oft bereits beim Anblick der gesamten ersten Seite sehr verunsichert und waren geneigt die Befragung lieber komplett abzulehnen, „um nichts falsch 
zu machen“. Da auch 577 Patienten ungültige Aussagen und nur 178 (24\%) die Tabelle korrekt genutzt haben ist die richtige Anwendung weiter zu optimieren. Es wurde leider oft nicht deutlich, dass es sich um jede Schmerzlokalisation während des aktuellen Aufenthalts handeln soll, unabhängig vom Grund der Aufnahme. Gleichzeitig wurde die sehr interessante Unterscheidungsmöglichkeit zwischen „ja“" (der Schmerz war vorhanden) und der Bewertung „schlimmster Schmerz“ überhaupt nicht getätigt, was zeigt, dass diese gewünschte Bewertung von den Patienten nicht umgesetzt wurde. In einigen Fällen wurde sogar die Spalte "schlimmster Schmerz" als ein "nein“ in Bezug auf die Schmerzkategorie interpretiert.

Hier muss daher deutlich nachgebessert werden, damit die Tabelle ihren Zweck sinnvoll erfüllen kann, denn eine inhaltlich korrekte Bewertung stellt einen großen Mehrwert für die klinische Arbeit dar. Die grundsätzliche Struktur der Tabelle sollte daher möglichst beibehalten werden. Als Lösung bietet sich zunächst eine umfangreiche Textreduktion an (Abbildung 22), um die Tabelle übersichtlicher zu gestalten. Dazu könnten in der Beschreibung die beiden Ankreuzfelder auf die Bezeichnung „Spalte 1 und 2“ umbenannt werden, um mit der Handlungsanweisung präziser zu werden. Es könnte so möglicherweise der Spielraum für eigene Interpretationen der Fragestellung reduziert werden, wodurch in der Folge die Fehlerrate verkleinert werden könnte. Durch diese Entlastung würde zudem die gesamte erste Seite des Fragebogens ansprechend überschaubar und weniger abschreckend dargestellt werden. 


\begin{tabular}{|c|c|c|}
\hline $\begin{array}{c}\text { Wählen Sie in der folgenden Tabelle in Spalte } 1 \text { alle die Schmerzarten-/orte aus, die } \\
\text { bei Ihnen } \text { HEUTE vorliegen. Wählen Sie im zweiten Schritt in Spalte } 2 \text { von den } \\
\text { markierten Schmerzen in Spalte 1, den für Sie Schlimmsten aus! }\end{array}$ & Spalte 1 & Spalte 2 \\
\hline Gesichtsschmerzen & D & 口 \\
\hline Kopfschmerzen & 口 & $\square$ \\
\hline Nackenschmerzen & $\square$ & $\bar{\square}$ \\
\hline Rücken- oder Kreuzschmerzen & $\square$ & $\square$ \\
\hline Mund-/ Halsschmerzen (z.B. beim Schlucken/Essen) & $\bar{\square}$ & $\bar{\square}$ \\
\hline Herzschmerzen (z.B. Angina pectoris) & $\square$ & $\square$ \\
\hline Schmerzen im Brustkorb (z.B. beim Husten, Atmen) & $\square$ & $\square$ \\
\hline Bauchschmerzen & $\square$ & $\square$ \\
\hline Leistenschmerzen & $\square$ & $\square$ \\
\hline Blasen- oder Nierenschmerzen & $\bar{\square}$ & $\bar{\square}$ \\
\hline Gelenk- oder Knochenschmerzen & $\square$ & $\bar{\square}$ \\
\hline Muskelschmerzen & $\square$ & 口 \\
\hline $\begin{array}{l}\text { Nervenschmerzen (z.B. Ischias, Nervenverletzungen oder } \\
\text { Polyneuropathie bei Zuckerkrankheit, Gürtelrose) }\end{array}$ & 口 & 口 \\
\hline Schmerzen bei Durchblutungsstörungen & 口 & 口 \\
\hline Schmerzen der Haut & $\square$ & $\square$ \\
\hline Wund- oder Narbenschmerzen & $\square$ & $\square$ \\
\hline Schmerz bei Untersuchung, Eingriff, Punktion, Behandlung & $\square$ & $\square$ \\
\hline Andere Schmerzen (bitte benennen): & $\square$ & $\square$ \\
\hline
\end{tabular}

Abbildung 22: Überarbeitete Liste der möglichen Schmerzentitäten (vgl. mit Abbildung 2)

\subsubsection{Veränderungen an Frage 8}

In der Projektphase wurde deutlich, dass das Layout der Frage wohl vergleichbar mit Frage 1 noch nicht ausreichend klar genug gewählt wurde, dass entweder die Skala oder die Alternativantwort auszuwählen waren. In einigen Fällen wurden sowohl die Skala als auch die Alternativantwort und damit widersprüchliche Antworten ausgewählt. Ein deutlicherer Zusammenhang zwischen beiden Antwortmöglichkeiten muss daher hergestellt werden, um solche Fehler zukünftig zu vermeiden.

Wahrscheinlich könnte eine Änderung der Position des Ankreuzfeldes etwas daran ändern, indem dieses, genau wie bei den Fragen $1 b$ und c, eingerückt wird (Abbildung 23). Es war auch einigen Patienten nicht möglich eine Aussage über ihre Zufriedenheit zu tätigen, weil sie angegeben haben subjektiv Schmerzen empfunden zu haben, aber keine Schmerzbehandlung erfolgt sei. Zum Teil wurde dies von den Patienten selbst begründet, indem sie das therapeutische Team darüber gar nicht erst informiert haben. In diesen Fällen sind alle bisherigen Antwortmöglichkeiten nicht wirklich zutreffend gewesen.

Eine solche Antwortoption kann damit erzielt werden, indem der Patient ankreuzen kann „Ich habe keine Schmerztherapie benötigt" bzw. „Ich hatte keine Schmerzen“ und „Ich 
hatte Schmerzen, brauchte bzw. wünsche aber keine Behandlung während des Aufenthaltes". Die Aussage „keine Schmerztherapie, da keine Schmerzen“ trifft einfach nicht bei jedem Patienten zu, da vielfach Schmerzen von den Patienten toleriert werden.

\section{Wie zufrieden waren Sie bisher mit Ihren Schmerzen während Ihrer Zeit im Krankenhaus bzw. mit Ihrer Schmerzbehandlung (z.B. Medikamente, Krankengymnastik oder Gespräche)?}

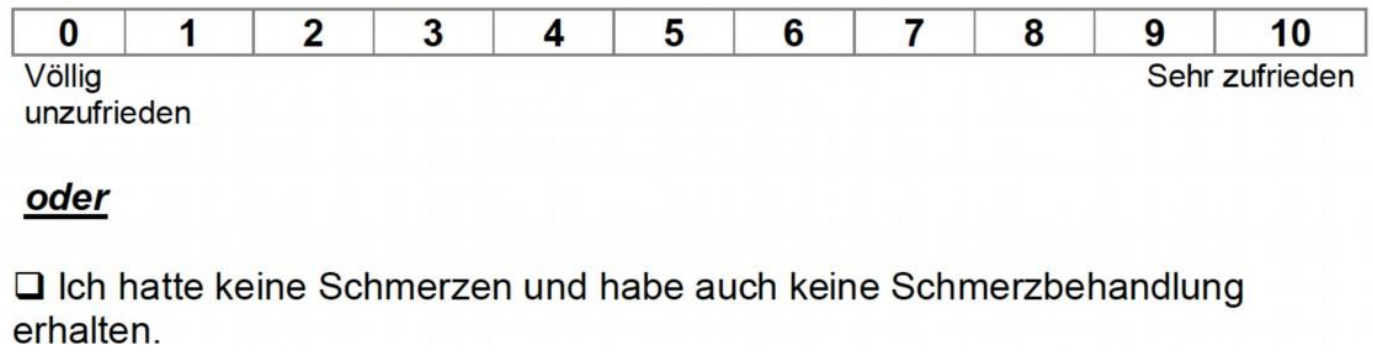

Abbildung 23: Überarbeitete Frage zur Patientenzufriedenheit

\subsubsection{Veränderungen an den Prozessparametern}

Trotz einer hohen Beteiligung an der Studie mit 74\% der Patienten, könnte diese Zahl noch durch kleinere Anpassungen verbessert werden. Durch eine Abänderung der zu unterschreibenden Einverständniserklärung, in der noch deutlicher der anonymisierte Aspekt der Befragung hervorgehoben wird (z. B. keine Erfassung von Namen, Adresse oder Geburtsdatum) und durch eine mögliche Anpassung des Befragungstages selbst, kann dieser Wert noch deutlich angehoben werden. Denn die größte Gruppe (14\%) der nicht erhebbaren Patientendaten fielen auf eine vorzeitige Entlassung vor dem dritten Aufnahmetag oder die Abwesenheit vom Patientenzimmer zurück. Zwar wurde auch von Patienten zurückgemeldet, dass bis zum dritten Aufnahmetag subjektiv noch keine spezifische Therapie eingeleitet worden sei, aber möglicherweise bietet sich hier doch an, den äquivalenten ersten Aufnahmetag aus QUIPS zu übernehmen. Besonders für Befragungen in Fachbereichen mit vielfach kurzer Krankenhausverweildauer für rein diagnostische Zwecke (z. B. in der Gastroenterologie oder Kardiologie) könnte dies hilfreicher sein. So wäre gleichzeitig eine noch engere Verzahnung zu QUIPS geschaffen.

Alternativ könnte eine Befragung auch am jeweiligen Entlassungstag stattfinden, wenn dieser bereits vor dem dritten Aufnahmetag liegen würde.

Von den bewussten Ablehnungen spielte eine Skepsis gegenüber der Anonymität eine immer wiederkehrende Rolle. Um diesen Patienten ein sichereres Gefühl und keinen Grund zur Skepsis zu geben, sollten diese Bedenken aufgegriffen und noch deutlicher 
schriftlich in der Einverständniserklärung etabliert werden. Auch wenn diese Bedenken im Rahmen von QUIKS unzutreffend sind, zeigen sie, wie verunsichert die Patienten in der heutigen Zeit im Umgang mit ihren sensiblen Daten sind. Solche verunsichernden Formulierungen sollten daher so gering wie möglich gehalten werden bzw. am besten gar nicht vorhanden sein.

Die Auswahlmöglichkeit „Patient ist verwirrt/hat Verständnisschwierigkeiten“ auf der ersten Fragebogenseite, warum eine Patientenbefragung nicht erfasst werden kann, sollte bearbeitet werden. Bisher wurde diese Option immer ausgewählt, wenn der Patient aus gesundheitlichen Gründen nicht befragt werden konnte. Hier bietet sich daher an eine zusätzliche eigene Rubrik „gesundheitliche Gründe“ einzufügen, da nicht jeder Patient als „verwirrt bzw. hat Verständnisschwierigkeiten“ zu bezeichnen ist, wenn er nur beispielsweise aufgrund eines reduzierten Allgemeinzustands nicht befragt werden konnte. Die bisherige Auswahl sollte aber dennoch in der Liste erhalten bleiben. Eine Angabe zu „Patient schläft bzw. ist sediert" war in der Praxis bisher nicht notwendig und könnten daher möglicherweise ganz aus dieser Rubrik gestrichen werden.

Zudem zeigten sich innerhalb der Prozessparameter vor allem im Bereich der jeweiligen Hauptdiagnose und den Auswahlmöglichkeiten der Medikation Schwierigkeiten.

Die Anwendbarkeit einer Wortdiagnoseliste als Benchmarking-Faktor zeigte sich allgemein als schwierig umzusetzen und könnte in seiner Gesamtheit noch einmal überdacht werden. Grundsätzlich muss hier vorab auch wieder die geringe Anzahl an teilnehmenden nicht repräsentativen Kliniken bei der Bewertung und Korrektur bedacht werden. Die Hauptdiagnose zeigt sich zwar bei allen Patienten als einziges sinnvoll vergleichbares Kriterium, die Umsetzung in Form der Liste ist jedoch in der aktuellen Projektphase nicht aussagekräftig umsetzbar gewesen. Eine möglicherweise besser praktikable Variante könnte die zukünftige Umsetzung der bereits oben angesprochenen Lösung für Frage 1 mittels Computer oder Tablet sein. Die Heterogenität der Hauptdiagnosen wird sich zweifelsohne nicht durch diese Variante lösen lassen, aber der Umfang der Wortdiagnoseliste ließe sich stark erweitern, ohne eine nicht mehr funktionelle Größe zu erreichen. Ein oberflächlicher Vergleich nur von Organsystemen als Obergruppen (z. B. Herz vs. Nervensystem) würde keine sinnvoll vergleichbare Basis abbilden, um eine ernsthafte Alterna- 
tive darstellen zu können. Als möglicherweise weitere sinnvolle, aber auch zeitaufwändige Alternative könnte jedoch auch angedacht werden, noch einmal zurück zur ICD-10Kodierung zu gehen und eine noch umfangreichere Sondierung der Kodierungen am jeweiligen Befragungstag in den bundesweit interessierten Kliniken durchzuführen. Im Bereich der Medikationen wurde die Liste bereits frühzeitig nach einer kleinen Stichprobe angepasst (Zweite Testphase), die dann in der Endfassung vervollständigt werden soll. Die grundsätzliche Erfassung zeigte hier in der Praxis auch wenig gravierende Probleme, wodurch in der Folge vorerst keine weiteren Veränderungen notwendig wurden. Der Fragepunkt „S6 - Spezielle Analgesieverfahren“ (z. B. eine PCIA) könnte jedoch nach der Projektphase komplett entfallen, da diese Möglichkeiten einer Schmerztherapie bei den konservativen (nicht operativen) Patienten aller beteiligten Kliniken nicht vorgekommen sind.

\subsection{Ausblick}

Nach erfolgreicher Auswertung der Ergebnisse stellt sich die Frage wie das zukünftige Benchmarking gestaltet sein soll. Ob nun beispielsweise eine Befragung aller Patienten oder nur der Patienten mit empfundenen Schmerzen aussagekräftiger ist und welcher Benchmarking-Faktor zugrunde gelegt wird, sollte in Folgeprojekten noch einmal hinterfragt und getestet werden.

Das aktuelle QUIKS-Modul wird derzeit im Kreise der kooperierenden und projektleitenden Kliniken konstruktiv beraten. Die in dieser Promotionsarbeit vorgeschlagenen möglichen Veränderungen oder Anpassungen werden dort im Konsens besprochen und gegebenenfalls dann auch umgesetzt. Es kann daher zum gegenwärtigen Zeitpunkt noch keine endgültige Fassung von QUIKS als Fazit der Projektphase für die klinische Einführung vorgestellt werden.

Alle abschließend getroffenen Anpassungen werden dann in der aktualisierten Verfahrensanweisung niedergeschrieben, damit eine einheitliche Anwendung in allen teilnehmenden Kliniken komplikationslos möglich ist. 


\section{$5 \quad$ Zusammenfassung}

Trotz umfangreicher Möglichkeiten für eine suffiziente Schmerztherapie klagen noch immer viel zu viele Patienten im Krankenhaus über nicht akzeptable Schmerzen. In einer groß angelegten Studie von Maier et al. (2010) konnten sogar signifikante Defizite in der Therapie nicht operativer Patienten aufgezeigt werden.

Zur stetigen Verbesserung der Qualität der Schmerztherapie werden heute im Bereich der postoperativen Schmerztherapie vielfach Benchmarking-Projekte wie QUIPS oder PAIN OUT eingesetzt, um eine subjektive Bewertung der erhaltenen Schmerztherapie aus Patientensicht zu erhalten. Diese beiden Benchmarking-Projekte konnten die Ergebnisse der Schmerztherapie nachweislich verbessern. Für konservativ behandelte Patienten gibt es zurzeit leider noch keine Benchmarking-Lösung. Mit dieser Promotionsarbeit wurde daher erstmals ein Projekt mit diesem Ziel an einem größeren Patientenkollektiv durchgeführt.

Entsprechend dem QUIPS-Projekt wurde das Benchmarking-Modul QUIKS entworfen, dass die Patienten entsprechend ihrer Aufnahmediagnose vergleichen soll. Sowohl die ergebnisbezogenen, als auch die prozessbezogenen Erfassungsparameter wurden inhaltlich und bzgl. ihrer Systematik und Erfassung möglichst nah an die bei QUIPS etablierte Methode angepasst. An drei großen deutschen Kliniken in Göttingen, Jena und Halle wurden im Zeitraum von April bis Oktober 2016 insgesamt 1317 konservativ behandelte Patienten am dritten Aufnahmetag anonymisiert befragt. Die Hauptdiagnose wurde mittels einer Wortdiagnoseliste erfasst, da sich in den voran gegangenen Testphasen keine einheitliche Deckung mit kodierten ICD-10-Diagnosen am Befragungstag präsentierte.

Einer Befragung haben 74\% der Patienten zugestimmt, die daraufhin den Fragebogen selbstständig oder in Form eines Interviews ausgefüllt haben. Um zu erfahren, wie viele Patienten im Befragungszeitraum Schmerzen empfunden haben, wurde die Frage nach dem Maximalschmerz (mit NRS > 0) als Indikator definiert. Insgesamt 63\% der Patienten machten auch auf der NRS eine entsprechende Angabe. In den Auswertungen musste berücksichtigt werden, dass das Auftreten und der Verlauf von Schmerzen im Vergleich zum operativen Bereich nicht eng mit einem auslösenden Ereignis verknüpft sein muss. Sie sind daher sehr komplex und zeigen eine stark individuell unterschiedliche Relevanz, auch im Wunsch nach einer Schmerzbehandlung auf. 
Während der Befragungen bildete sich schnell ab, dass das konservativ behandelte Patientenkollektiv sehr heterogen gestreut war und sich eine Vergleichbarkeit anhand der Diagnose als sehr schwierig gestalten könnte. Dennoch konnten den Patienten mit der aktuellen Wortdiagnoseliste in 94\% eine klar definierte Hauptdiagnose zugeordnet werden. Allein 26\% der Hauptdiagnosen waren bisher noch nicht in der Wortdiagnoseliste aufgeführt und wurden unter der Rubrik „sonstige klar definierte Hauptdiagnose“ namentlich manuell hinzugefügt. Die Heterogenität zeigte sich besonders darin, dass die Hauptdiagnosen sehr breit gefächert waren und selten anteilig über 2\% lagen. Die häufigste aufgelistete Hauptdiagnose war mit nur knapp 9\% der Schlaganfall. Die Wortdiagnoseliste in der aktuellen Form zeigt sich daher als noch nicht ganz ausgereift und sinnvoll einsetzbar. Entsprechende Änderungsvorschläge wurden hierzu bereits gemacht.

Bei der Auswertung der Ergebnisse wurden zur besseren Vergleichbarkeit mit QUIPS ähnliche Verfahren angewendet. So wurde zum einen zur Variablenreduktion eine Faktorenanalyse, sowie zur Analyse der Reliabilität die Bestimmung des Cronbachs $\alpha$ durchgeführt. Beide Verfahren zeigten dabei vergleichbar positive Ergebnisse. In der Auswertung mithilfe der COSMIN-Checkliste wurden die jeweils wesentlichen Aspekte erfüllt. Im ersten Versuch einer qualitativen Auswertung zeigte sich im Mann-Whitney-U-Test und im Studienvergleich, dass der QUIKS Fragebogen durchaus in der Lage ist, Unterschiede zwischen verschiedenen Patientengruppen (klassifiziert anhand der Wortdiagnosen von QUIKS) im Sinne einer klinischen Validität nachzuweisen, wodurch die grundsätzliche Einsetzbarkeit gewährleistet ist und eine Integration in das QUIPS-Projekt langfristig komplikationslos ablaufen könnte. 


\section{$6 \quad$ Anhang}

\subsection{QUIKS-Fragebogen}

Qualitatsverbesserung In der Schmerztheraple konservattver (nicht-operativer) Patlenten (QUIKS) - Ergebnis-Fragebogen

Sehr geehrte Patientin, sehr geehrter Patient, wir führen an dieser Klinik eine Befragung zu gesundheitlichen Beschwerden im Rahmen stationärer Behandlungen durch. Wir möchten Sie daher höflich bitten, die folgenden Fragen zu beantworten. Die Datenerhebung ist freiwillig. Ihre persönlichen Angaben werden den behandelnden Ärztinnen und Ärzten und dem Pflegepersonal nicht zugänglich gemacht und nach Datenerfassung anonymisiert, d.h. ohne Name oder Geburtsdatum erfasst. Falls Sie sich nicht zu einer Teilnahme entschließen können, hat das keine Auswirkungen auf ihre weitere Behandlung.

Kreuzen Sie bitte auf den Skalen jeweils die Zahl an, die für thren Schmerz zutrifft $\left(0^{\circ}\right.$ bedeutet Schmerzfreilheit und _ $10^{\circ}$ stärkster vorstellbarer Schmerz).

1a. Hatten Sie ständige Schmerzen, die drei Monate oder länger andauerten, bevor Sie ins Krankenhaus gekommen sind?

$$
\text { 口 Ja Dein }
$$

1b. Falls ja, wie stark waren diese Schmerzen meistens?

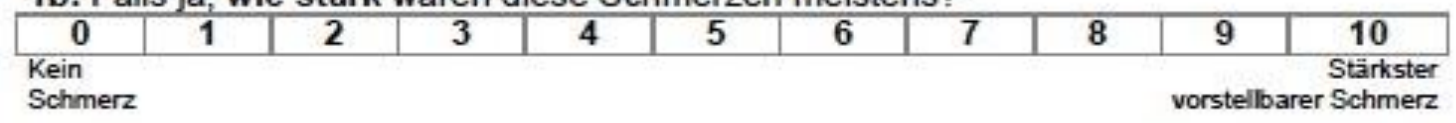

1c. Falls ja, waren diese Schmerzen Grund für die Krankenhausaufnahme?

$\square$ ja $\square$ nein $\square$ weiß nicht

Bei den folgenden Fragen geht es um Ihre Schmerzen, die während des Aufenthalts im Krankenhaus bestanden:

2a. Haben Sie bisher während Ihres Aufenthaltes im Krankenhaus an einer oder mehreren der folgenden Schmerzarten gelitten?

\begin{tabular}{|l|c|c|}
\hline $\begin{array}{c}\text { Bitte kreuzen Sie in der ersten Spalte die Kästchen der Schmerzarten an, die bei } \\
\text { Ihnen zutreffen. Geben Sie bitte auch die Schmerzen an, die eventuell in keinem } \\
\begin{array}{c}\text { Zusammenhang mit ihrer aktuellen Erkrankung stehen. Dann kreuzen Sie in der } \\
\text { zweiten Spalte an, welche der Schmerzarten für Sie am schlimmsten war/ist. }\end{array}\end{array}$ & $\begin{array}{c}\text { Ja } \\
\text { (hier alles } \\
\text { ankreuzen, } \\
\text { was bei lhnen } \\
\text { zutriffi) }\end{array}$ & $\begin{array}{c}\text { Schlimmste } \\
\text { Schmerz } \\
\text { (hier bitte nur } \\
\text { eines } \\
\text { ankreuzen') }\end{array}$ \\
\hline Gesichtsschmerzen & $\square$ & $\square$ \\
\hline Kopfschmerzen & $\square$ & $\square$ \\
\hline Nackenschmerzen & $\square$ & $\square$ \\
\hline Rücken- oder Kreuzschmerzen & $\square$ & $\square$ \\
\hline Mund-/Halsschmerzen (z.B. beim Schlucken/Essen) & $\square$ & $\square$ \\
\hline Herzschmerzen (z.B. Angina pectoris) & $\square$ & $\square$ \\
\hline Schmerzen im Brustkorb (z.B. beim Husten, Atmen) & $\square$ & $\square$ \\
\hline Bauchschmerzen & $\square$ & $\square$ \\
\hline Leistenschmerzen & $\square$ & $\square$ \\
\hline Blasen- oder Nierenschmerzen & $\square$ & $\square$ \\
\hline Gelenk- oder Knochenschmerzen & $\square$ & $\square$ \\
\hline Muskelschmerzen & $\square$ & $\square$ \\
\hline $\begin{array}{l}\text { Nervenschmerzen (z.B. Ischias, Nervenverletzungen oder } \\
\text { Polyneuropathie bei Zuckerkrankheit, Gürtelrose) }\end{array}$ & $\square$ & $\square$ \\
\hline Schmerzen bei Durchblutungsstörungen & $\square$ & $\square$ \\
\hline Wund- oder Narbenschmerzen & $\square$ & $\square$ \\
\hline Schmerz bei Untersuchung, Eingriff, Punktion, Behandlung & $\square$ & $\square$ \\
\hline Andere Schmerzen (bitte benennen): & $\square$ & $\square$ \\
\hline
\end{tabular}


Qualtatsverbesserung in der Schmerztheraple konservattver (nicht-operattver) Patlenten (QUiKS) - Ergebnis-Fragebogen

Kreuzen Sie bitte auf den Skalen jeweils die Zahl an, die für thren Schmerz zutrifft $\left(0^{\circ}\right.$ bedeutet Schmerzfreiheit und ${ }_{-10^{\circ}}$ stärkster vorstellbarer Schmerz).

2b. Wie stark waren Ihre Schmerzen bei der Aufnahme ins Krankenhaus?

\begin{tabular}{|c|c|c|c|c|c|c|c|c|c|c|}
\hline 0 & 1 & 2 & 3 & 4 & 5 & 6 & 7 & 8 & 9 & 10 \\
\hline $\begin{array}{l}\text { Kein } \\
\text { Schmer }\end{array}$ & & & & & & & & & & $\begin{array}{l}\text { Stärkste } \\
\text { r Schmen }\end{array}$ \\
\hline
\end{tabular}

2c. Bitte kreuzen Sie hier den stärksten Schmerz an, den Sie seit Ihrem Krankenhausaufenthalt bei schmerzhaften Prozeduren/Maßnahmen (z.B. Untersuchung, Eingriff, Punktion, Behandlung) hatten.

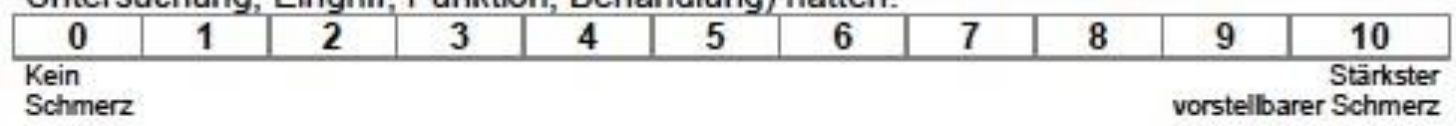

Die nächsten drei Fragen beziehen sich darauf, wie Ihre Schmerzen innerhalb der letzten 24 Stunden waren:

3a. Bitte kreuzen Sie hier den Schmerz an, den Sie innerhalb der letzten 24 Stunden hatten, wenn Sie sich ruhig verhalten haben.

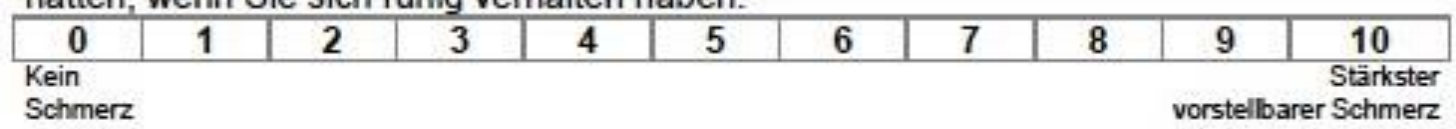

3b Wie stark waren Ihre Schmerzen innerhalb der letzten 24 Stunden, wenn Sie sich belastet haben, zum Beispiel bei Mobilisierung, Bewegen, Waschen, Husten, Durchatmen?

\begin{tabular}{l|l|l|l|l|l|l|l|l|c|c|}
\hline $\mathbf{0}$ & $\mathbf{1}$ & $\mathbf{2}$ & $\mathbf{3}$ & $\mathbf{4}$ & $\mathbf{5}$ & $\mathbf{6}$ & $\mathbf{7}$ & $\mathbf{8}$ & $\mathbf{9}$ & $\mathbf{1 0}$ \\
\hline $\begin{array}{l}\text { Kein } \\
\text { Schmerz }\end{array}$
\end{tabular}

3c. Bitte kreuzen Sie hier den stärksten Schmerz an, den Sie innerhalb der letzten $24 \mathrm{~h}$ hatten.

\begin{tabular}{l|l|l|l|l|l|l|l|l|l|c|}
\hline 0 & 1 & 2 & 3 & 4 & 5 & 6 & 7 & 8 & 9 & 10 \\
\hline $\begin{array}{ll}\text { Kein } \\
\text { Schmerz }\end{array}$
\end{tabular}

Die nächsten vier Fragen beziehen sich darauf, ob bestimmte Tätigkeiten oder Ihre Stimmung innerhalb der letzten 24 Stunden durch den Schmerz beeinträchtigt waren. Mit , beeinträchtigt" ist gemeint: die Tātigkeit ist unmöglich oder nur unter großer Mühe möglich. Waren Sind Sie durch die Schmerzen beeinträchtigt:

4a. bei Bewegung?

$$
\text { 口 Ja a Nein }
$$

4b. beim Husten oder tiefen Luftholen?

口 Ja $\square$ Nein

4c. beim Schlafen?

$$
\text { 口 Ja } \square \text { Nein }
$$

4d. in Ihrer Stimmung?

व Ja $\quad$ Nein


Qualitatsverbesserung in der Schmerztherapie konservattver (nicht-operativer) Pattenten (QUIKS) - Ergebnis-Fragebogen

Die nächsten 4 Fragen beziehen sich auf Ihren Allgemeinzustand in den letzten 24 Stunden:

5a. Haben Sie sich sehr müde gefühlt?
口 Ja
口 Nein

5b. Haben Sie unter Übelkeit gelitten?
口 Ja
a Nein

5c. Haben Sie unter Schwindel gelitten?
口 Ja
a Nein

5d. Leiden Sie derzeit unter Verstopfung?

口 Ja $\square$ Nein

6. Wurden Sie über die verschiedenen Möglichkeiten Ihrer Schmerztherapie informiert?
a Ja
ㅁ Nein

7. Hätten Sie gewünscht, mehr Mittel gegen Schmerzen zu bekommen?
口 Ja
a Nein

8. Wenn Sie Schmerzen während Ihres Aufenthaltes im Krankenhaus hatten bzw. Schmerztherapie erhalten haben (z.B. Medikamente, Schmerzsalben, Schmerzkatheter, Krankengymnastik, Wärme, Kälte, Gespräch usw.) kreuzen Sie bitte an, wie zufrieden Sie mit dem Ergebnis der Behandlung Ihrer Schmerzen sind.

\begin{tabular}{|l|l|l|l|l|l|l|l|l|l|l|}
\hline $\mathbf{0}$ & $\mathbf{1}$ & $\mathbf{2}$ & $\mathbf{3}$ & $\mathbf{4}$ & $\mathbf{5}$ & $\mathbf{6}$ & $\mathbf{7}$ & $\mathbf{8}$ & $\mathbf{9}$ & 10 \\
\hline $\begin{array}{l}\text { Völlig } \\
\text { unzufrieden }\end{array}$
\end{tabular}

- Ich hatte keine Schmerzursache/nie Schmerzen und habe auch keine Schmerzbehandlung erhalten. 


\subsection{QUIPS-Fragebogen}

Sehr geehrte Patientin, sehr geøhrter Patient,

\section{QUIPS Ergebnis-Fragebogen}

wir fahren an dieser Klinik eine Befragung zu gesundhettichen Beschwerden nach Operatianen durch. Wir möchten Sie daher hölich bitten, die folgenden Fragen zu beantworten. Die Detenerhebung ist freivillig. thre Angaben werden den behandelnden Arztinnen und Arzten und dem Pflegepersonal nicht zuganglich gemacht und nach Dateneingabe anconymisiert, d. h. Ihr Name wird geloscht. Falls Sie sich nicht zu einer Telnehme entschließen können, hat das keine Auswirkungen auf itre weitere Behandlung

E1: Wurden Sie vor der Operation ausreichend uber die Moglichkeiten der Schmerztherapie aufgeklärt?

$\square . \mathrm{Ja}$, nur allgemein $\square . \mathrm{Ja}$, auch uber spezielle Schmerztherapieverfahren $\square$ Nein

E2: Schmerz bei Belastung

Wie stark weren Itre Schmerzen seit der Operation, wenn Sie sich belastet haben, zum Beispiel bei

Mobilisierung. Bewegen, Waschen, Husten, Durchatmen?

Kreuzen Sie bitte die Zahl aut der Skala an, die für Inren Belastungsschmerz zutritt $\left\{0^{*}\right.$ bedeutet Schmarzfreiheit und $10^{5}$ stärkster vorstallbarer Schmerz)

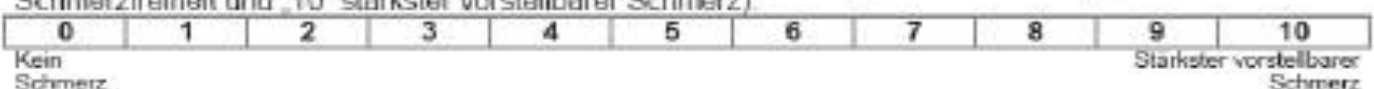

E3: Maximalschmerz seit der Operation

Wie stark waren bisher lihre starksten Schmerzen seit der Operation?

Kreuzen Sie bitte wioder die Zahl aut der Skala an, die fur Ihren starksten Schmerz zutrift

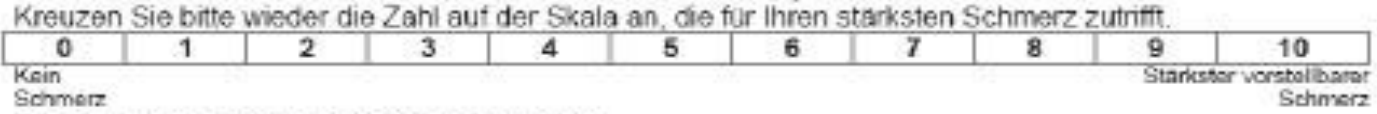

E4: Geringeter Schmerz seit der Operation

Wie stark waren bisher thre geringsten Schmerzen seit der Operation?

Kreuzen Sie bitte wieder die Zahl auf der Skala an, de für Ihren geringsten Schmerz zutrift.

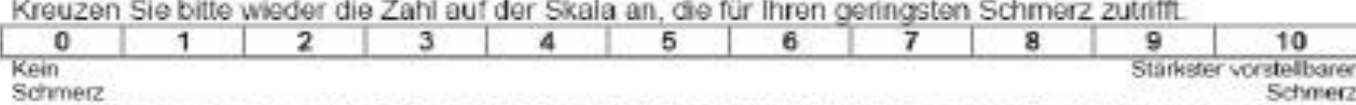

Die nächsten vier Fragen beziehen sich darauf, co bestimmte Tatigkeiten oder Ihre Stimmung seit der.

Operation durch den Schmerz beeintrachtigt sind Mit beeintrachtigt" ist gemeint: de Tatigheit ist

unmöglich oder nur unter großer Mühe moglich.

E5: Sind Sie durch die Schmerzen in threr Mobilität brw. Bewegung beeintrăchtigt?

口. Ja D Nein

E6: Sind Sie durch die Schmerzen beim Husten oder tiefen Luftholen beeinträchtigt?

(1) Ja

E7: Sind Sie durch die Schmerzen heute Nacht aufgewacht?

口 Ja $\square$ Nein

E8: Sind Sie durch die Schmerzen in Ihrer Stimmung beeinträchtigt?

口. Ja $\square$ Nein

E9: Hätten Sie gewanscht, mehr Mittel gegen Schmerzen zu bekommen?

口. Ja $\square$ Nein

E10: Haben Sie sich seit der Operation sehr mude geruhlt?

口 Ja D Nein

E11: Haben Sie seit der Operation unter Übelkeit gelitten?

口 la $\square$ Nein

E12: Haben Sie seit der Operation erbrochen?

7. b 0 Nein

E13: Wie zufrieden sind Sie mit der Schmerzbehandlung seit der Operation?

Kreuzen Sie bitte die Zahil an, die fur Ihre Zufriedenheit zutrifft

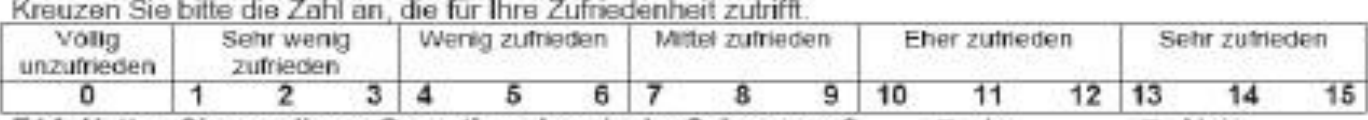

E14: Hatten Sie vor dieser Operation chronische Schmerzen? 7 ja Nein

E15: Falls ja, wie stark waren diese Schmerzen?

Kreuzen Sie bitte die Zahl auf der Skala an, die fur Ihre Schmerzen zumin $\left\{0^{\circ}\right.$ bedeutet Schmerzfreiheit und $10^{\circ}$ starkster vorstellbarer Schimerz)

\begin{tabular}{|c|c|c|c|c|c|c|c|c|c|c|}
\hline 0 & 1 & 2 & 3 & 4 & 5 & 6 & 7 & 8 & 9 & 10 \\
\hline Kein \\
Schinerz
\end{tabular}




\subsection{PAIN-OUT-Fragebogen}

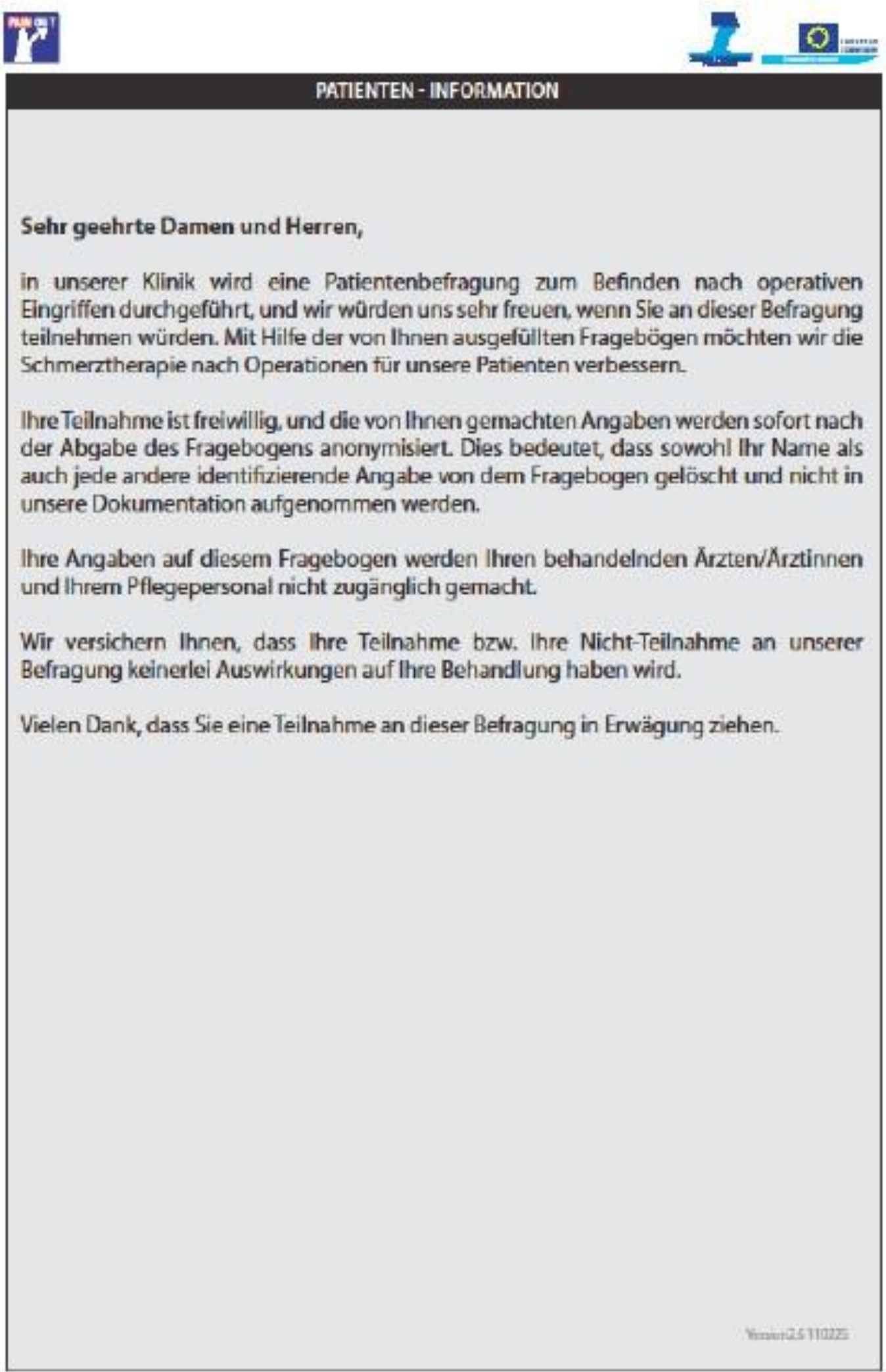


Bei den folgenden Fragen geht es um lhren Schmerz seit lhrer Operation.

P1. Bitte kreuzen Sie hier den stärksten Schmerz an, den Sie seit lhrer Operation hatten:

\begin{tabular}{|c|c|c|c|c|c|c|c|c|c|c|}
\hline 0 & 1 & 2 & 3 & 4 & 5 & 6 & 7 & 8 & 9 & 10 \\
\hline
\end{tabular}

P2. Bitte kreuzen Sie hier den geringsten Schmerz an, den Sie seit lhrer Operation hatten:

\begin{tabular}{|c|r|c|c|c|c|c|c|c|c|c|}
\hline 0 & 1 & 2 & 3 & 4 & 5 & 6 & 7 & 8 & 9 & 10 \\
\hline
\end{tabular}

P3. We oft hatten Sie seit threr Operation starke Schmerzen?

Bitte schătzen Sie den Zeitanteil, in dem Sie unter starken Schmerzen geItten haben, und kreisen Sie den Wert ein:

\begin{tabular}{|c|c|c|c|c|c|c|c|c|c|c|}
\hline $0 \%$ & $10 \%$ & 2096 & 3096 & $40 \%$ & $50 \%$ & $60 \%$ & $70 \%$ & 8096 & $90 \%$ & $100 \%$ \\
\hline
\end{tabular}

P4. Kreisen Sie den Wert ein, der am besten beschreibt, wie sehr Schmerzen Sie seit lhrer Operation bei den falgenden Aktivitäten beointröchtigt habon, brw. wie sehr diexe Aktivitäten durch Schmerzen vorhindert wurdon:

a. Aktivitäton im Bott wiez. B. umdrehen, aufsetzen, Position wechseln:

\begin{tabular}{|c|c|c|c|c|c|c|c|c|c|c|}
\hline 0 & 1 & 2 & 3 & 4 & 5 & 6 & 7 & 8 & 9 & 10 \\
\hline
\end{tabular}
keine Beeinträchtigung

b. Thef einatmen oder husten:

\begin{tabular}{|c|c|c|c|c|c|c|c|c|c|c|}
\hline 0 & 1 & 2 & 3 & 4 & 5 & 6 & 7 & 8 & 9 & 10 \\
koine Beointrachtigung
\end{tabular}
c. Schlafen:

\begin{tabular}{|c|c|c|c|c|c|c|c|c|c|c|}
\hline 0 & 1 & 2 & 3 & 4 & 5 & 6 & 7 & 8 & 9 & 10 \\
\hline kaine & trä & ung & & & & & & \multicolumn{3}{|c|}{ völlige Bevinträchtigung } \\
\hline
\end{tabular}

Falls jo, wie sehr haben Schmerzen Sic bei Aktivitäten außerholb des Bettes wic z. B. gchen, auf einem Stuhl sitzen, am Waschbecken stehen beeinträchtigt, bzw. wie schr wurden diese Alktivitaiten durch 5 chmerzen verhindert?

\begin{tabular}{|c|c|c|c|c|c|c|c|c|c|c|}
\hline 0 & 1 & 2 & 3 & 4 & 5 & 6 & 7 & 8 & 9 & 10 \\
\hline
\end{tabular}




\section{PATIENTEN - FRAGEBOGEN}

P5. Schmerz kann unsere Stimmung und unsere Gefühie beeinflussen.

Bitte kreisen Sie den Wert ein, der arn besten widerspiegelt, wie sehr Schmerzan seit lher Operation die folgonden Gefühlo bowirkt haber:

a. Verunsicharung

\begin{tabular}{|c|c|c|c|c|c|c|c|c|c|c|}
\hline 0 & 1 & 2 & 3 & 4 & 5 & 6 & 7 & 8 & 9 & 10 \\
\hline
\end{tabular}
gar nicht

b. Hilflosigkeit

\begin{tabular}{|c|c|c|c|c|c|c|c|c|c|c|}
\hline 0 & 1 & 2 & 3 & 4 & 5 & 6 & 7 & 8 & 9 & 10 \\
\hline \\
gar nicht
\end{tabular}

P6. Haben Sie eine der folgenden Nebenwirkungen seit threr Operation beobachtet? Bitte kreisen Sie bei Nein " 0 " ein; bei Ja, bitte den einen Wert, der am besten die Intensitat der Nebenwirkung widerspicgelt:

\section{a. Obelkeit}

\begin{tabular}{|c|c|c|c|c|c|c|c|c|c|c|}
\hline 0 & 1 & 2 & 3 & 4 & 5 & 6 & 7 & 8 & 9 & 10 \\
\hline
\end{tabular}

\section{b. Benommenheit}

\begin{tabular}{|c|c|c|c|c|c|c|c|c|c|c|}
\hline 0 & 1 & 2 & 3 & 4 & 5 & 6 & 7 & 8 & 9 & 10 \\
\hline
\end{tabular}

c. Juckrain

\begin{tabular}{l|l|l|l|l|l|l|l|l|l|l|}
\hline 0 & 1 & 2 & 3 & 4 & 5 & 6 & 7 & 8 & 9 & 10 \\
\hline
\end{tabular}

d. Schwindel

\begin{tabular}{|c|c|c|c|c|c|c|c|c|c|c|}
\hline 0 & 1 & 2 & 3 & 4 & 5 & 6 & 7 & 8 & 9 & 10 \\
\hline
\end{tabular}

P7. We grol war seit der Operation lhre Schmerzlinderung?

Bitte kreisen Sie den einen Wert ein, der am besten widerspiegelt, wie sehr lhr Schmerz aufgrund der kombinierten Schmerztherapie (medikamentose und nicht-medikamentose Behandlungl gelindert wurde:

\begin{tabular}{|c|c|c|c|c|c|c|c|c|c|c|}
\hline $0 \%$ & $10 \%$ & $20 \%$ & $30 \%$ & $40 \%$ & $50 \%$ & $60 \%$ & $70 \%$ & $80 \%$ & $90 \%$ & $100 \%$ \\
\hline
\end{tabular}

P8. Hätten Siesich MEHR Schmorztherapie gewonscht, als Sie erhalten haben?
$\square$ Ja
$\square$ Nein

P9. Wunden Sie über die verschiedenen Möglichkeiten Ihrer Schmerxtherapie informiert?
$\square$ Ja
$\square$ Nein 
P10. Wurden Sie in dem won thnen gewanschten MaB an Entscheidungen zu lhrer Schmerzthorapie boteiligt?

\begin{tabular}{|c|c|c|c|c|c|c|c|c|c|c|}
\hline 0 & 1 & 2 & 3 & 4 & 5 & 6 & 7 & 8 & 9 & 10 \\
gar nicht
\end{tabular}
völlig ausreichend

P11. Bätte kreisen Sie ein, wie zufriedan Sie mit dern Ergebnis lharer Schmerztherapie seit lhrer Operation sind:

\begin{tabular}{|c|c|c|c|c|c|c|c|c|c|c|}
\hline 0 & 1 & 2 & 3 & 4 & 5 & 6 & 7 & 8 & 9 & 10 \\
\hline
\end{tabular}
extrem unzufrieden

P12. Haben Sie nicht-medikamentöse Methoden zur Schmerzlinderung benutzt oder erhalten?

$$
\text { ロ la } \square \text { Nein }
$$

Falls ja, welche?

口Kühlkcmpressen

$\square$ Meditation $\square$ tiefes Atmen

口Warme

$\square$ Akupunktur

口 Beten

口Gespräche mit medizinischem Personal

$\square$ Gespräche mit Freunden oder Verwandten

$\square$ TENS (Transkutane elektrische Nervenstimulation)

$\square$ Ablenkung (wie 2. B. fernsehen, Musik hören, lesen)

$\square$ Sonstiges (bitte beschreiben):

P13. Hatten Sie ständige Schmerzen, die drei Monate oder länger andauerten, bevor Sie wegen dieser Operation ins Krankenhaus gekommen sind?

$\square$ Ja

a. Falls ja wie stark waren diese Sdhmeraen meistens?

Bitte kreisen Sie den Wert ein, der lhre Schmerzintensitat am besten widerspiegelt.

\begin{tabular}{|l|l|l|l|l|l|l|l|l|l|l|}
\hline 0 & 1 & 2 & 3 & 4 & 5 & 6 & 7 & 8 & 9 & 10 \\
\hline
\end{tabular}

kein Schmerz schlimmstmöglicher Schmerz

1. Falls ja, wo traten diese ständigen Schmerzen auf?

$\square$ an der Körperstelle, die coperiert wurde $\square$ an einer anderen Körperstelle

$\square$ beides (Operationsstelle und waanders)

\section{Vielen Dank für lhre Teilnahme}

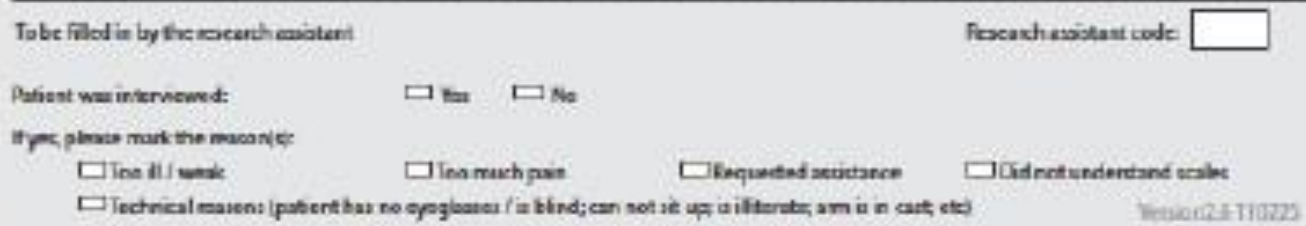




\subsection{COSMIN-4-Punkte-Skala}

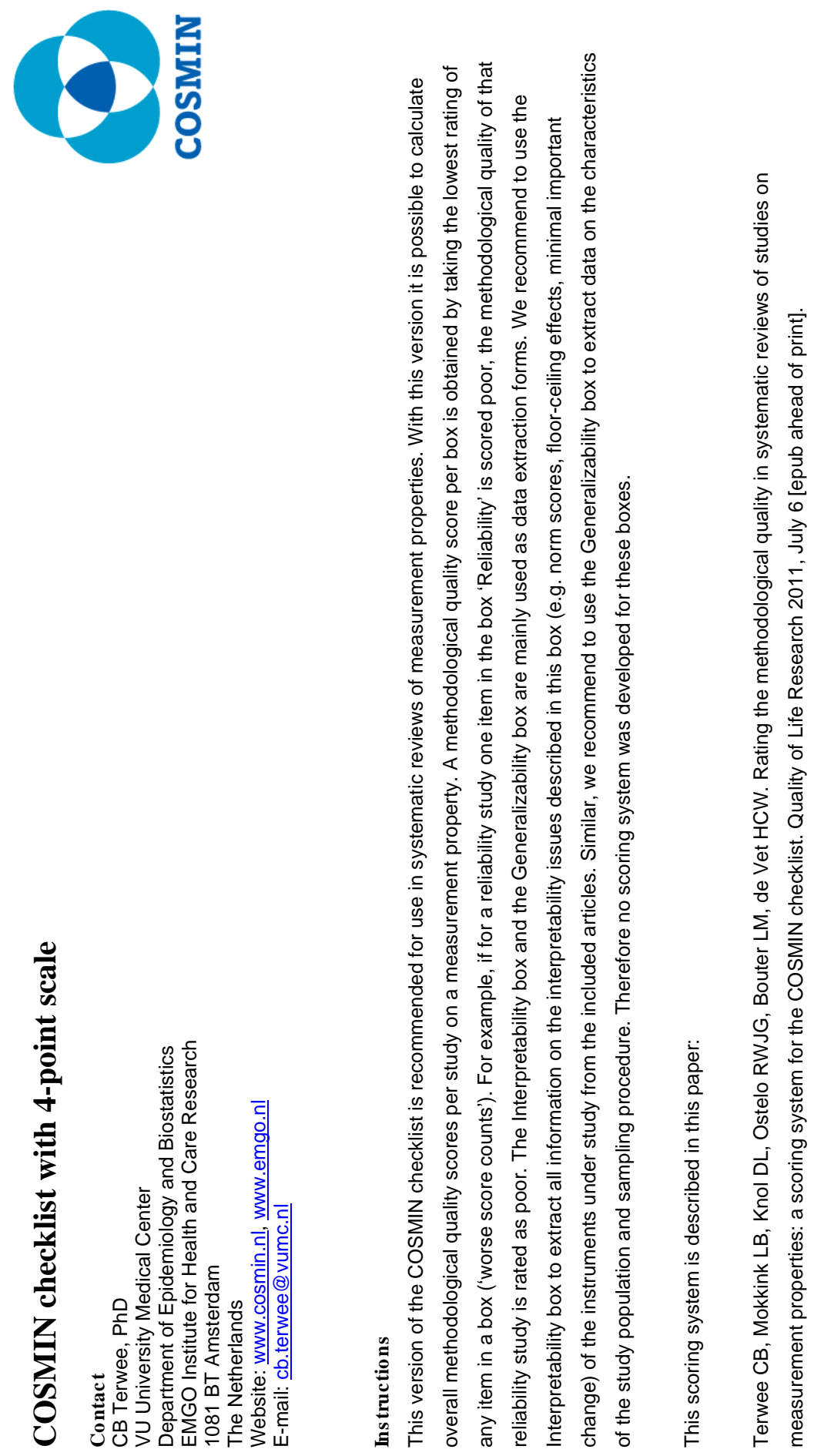




\begin{tabular}{|c|c|c|c|c|c|}
\hline \multicolumn{6}{|c|}{ Box A. Internal consistency } \\
\hline & & excellent & good & fair & poor \\
\hline \multicolumn{6}{|c|}{ Design requirements } \\
\hline & Was the percentage of missing items given? & $\begin{array}{l}\text { Percentage of } \\
\text { missing items } \\
\text { described }\end{array}$ & $\begin{array}{l}\text { Percentage of } \\
\text { missing items } \\
\text { NOT described }\end{array}$ & & \\
\hline & Was there a description of how missing items were handled? & $\begin{array}{l}\text { Described how } \\
\text { missing items } \\
\text { were handled }\end{array}$ & $\begin{array}{l}\text { Not described but } \\
\text { it can be deduced } \\
\text { how missing items } \\
\text { were handled }\end{array}$ & $\begin{array}{l}\text { Not clear how } \\
\text { missing items } \\
\text { were handled }\end{array}$ & \\
\hline 4 & Was the sample size included in the internal consistency analysis adequate? & $\begin{array}{l}\text { Adequate sample } \\
\text { size }(\geq 100)\end{array}$ & $\begin{array}{l}\text { Good sample size } \\
(50-99)\end{array}$ & $\begin{array}{l}\text { Moderate sample } \\
\text { size }(30-49)\end{array}$ & $\begin{array}{l}\text { Small sample } \\
\text { size }(<30)\end{array}$ \\
\hline 5 & $\begin{array}{l}\text { Was the unidimensionality of the scale checked? i.e. was factor analysis or IRT } \\
\text { model applied? }\end{array}$ & $\begin{array}{l}\text { Factor analysis } \\
\text { performed in the } \\
\text { study population }\end{array}$ & $\begin{array}{l}\text { Authors refer to } \\
\text { another study in } \\
\text { which factor } \\
\text { analysis was } \\
\text { performed in a } \\
\text { similar study } \\
\text { population }\end{array}$ & $\begin{array}{l}\text { Authors refer to } \\
\text { another study in } \\
\text { which factor } \\
\text { analysis was } \\
\text { performed, but not } \\
\text { in a similar study } \\
\text { population }\end{array}$ & $\begin{array}{l}\text { Factor analysis } \\
\text { NOT performed } \\
\text { and no } \\
\text { reference to } \\
\text { another study }\end{array}$ \\
\hline 6 & Was the sample size included in the unidimensionality analysis adequate? & $\begin{array}{l}7^{*} \text { \#items and } \\
\geq 100\end{array}$ & $\begin{array}{l}5^{\star} \# \text { \#items and } \\
\geq 100 \text { OR } 6-7^{\star} \\
\text { \#items but }<100\end{array}$ & $\begin{array}{l}5^{*} \text { \#items but } \\
<100\end{array}$ & $<5^{\star} \#$ \#items \\
\hline 7 & $\begin{array}{l}\text { Was an internal consistency statistic calculated for each (unidimensional) } \\
\text { (sub)scale separately? }\end{array}$ & \begin{tabular}{|l|} 
Internal \\
consistency \\
statistic calculated \\
for each subscale \\
separately
\end{tabular} & & & \begin{tabular}{|l} 
Internal \\
consistency \\
statistic NOT \\
calculated for \\
each subscale \\
separately
\end{tabular} \\
\hline 8 & Were there any important flaws in the design or methods of the study? & $\begin{array}{l}\text { No other important } \\
\text { methodological } \\
\text { flaws in the design } \\
\text { or execution of the } \\
\text { study }\end{array}$ & & $\begin{array}{l}\text { Other minor } \\
\text { methodological } \\
\text { flaws in the design } \\
\text { or execution of the } \\
\text { study }\end{array}$ & $\begin{array}{l}\text { Other important } \\
\text { methodological } \\
\text { flaws in the } \\
\text { design or } \\
\text { execution of the } \\
\text { study }\end{array}$ \\
\hline \multicolumn{6}{|c|}{ Statistical methods } \\
\hline 9 & $\begin{array}{l}\text { for Classical Test Theory (CTT), continuous scores: Was Cronbach's alpha } \\
\text { calculated? }\end{array}$ & $\begin{array}{l}\text { Cronbach's alpha } \\
\text { calculated }\end{array}$ & & $\begin{array}{l}\text { Only item-total } \\
\text { correlations } \\
\text { calculated }\end{array}$ & $\begin{array}{l}\text { No Cronbach's } \\
\text { alpha and no } \\
\text { item-total } \\
\text { correlations } \\
\text { calculated }\end{array}$ \\
\hline 10 & for $\mathrm{CTT}$, dichotomous scores: Was Cronbach's alpha or KR-20 calculated? & $\begin{array}{l}\text { Cronbach's alpha } \\
\text { or KR-20 } \\
\text { calculated }\end{array}$ & & $\begin{array}{l}\text { Only item-total } \\
\text { correlations } \\
\text { calculated }\end{array}$ & $\begin{array}{l}\text { No Cronbach's } \\
\text { alpha or KR-20 } \\
\text { and no item- } \\
\text { total correlations } \\
\text { calculated }\end{array}$ \\
\hline 11 & $\begin{array}{l}\text { for IRT: Was a goodness of fit statistic at a global level calculated? E.g. } X^{2} \text {, reliability } \\
\text { coefficient of estimated latent trait value (index of (subject or item) separation) }\end{array}$ & $\begin{array}{l}\text { Goodness of fit } \\
\text { statistic at a global } \\
\text { level calculated }\end{array}$ & & & $\begin{array}{l}\text { Goodness of fit } \\
\text { statistic at a } \\
\text { global level NOT } \\
\text { calculated }\end{array}$ \\
\hline
\end{tabular}

NB. Item 1 is used to determine whether internal consistency is relevant for the instrument under study. It is not used to rate the quality of the study.

\begin{tabular}{|c|c|c|c|c|c|}
\hline \multirow{2}{*}{\multicolumn{2}{|c|}{ Design requirements }} & excellent & good & fair & poor \\
\hline & & & & & \\
\hline 1 & Was the percentage of missing items given? & $\begin{array}{l}\text { Percentage of } \\
\text { missing items } \\
\text { described }\end{array}$ & $\begin{array}{l}\text { Percentage of } \\
\text { missing items } \\
\text { NOT described }\end{array}$ & & \\
\hline 2 & Was there a description of how missing items were handled? & $\begin{array}{l}\text { Described how } \\
\text { missing items } \\
\text { were handled }\end{array}$ & $\begin{array}{l}\text { Not described but } \\
\text { it can be deduced } \\
\text { how missing items } \\
\text { were handled }\end{array}$ & $\begin{array}{l}\text { Not clear how } \\
\text { missing items } \\
\text { were handled }\end{array}$ & \\
\hline 3 & Was the sample size included in the analysis adequate? & $\begin{array}{l}\text { Adequate sample } \\
\text { size }(\geq 100)\end{array}$ & $\begin{array}{l}\text { Good sample size } \\
(50-99)\end{array}$ & $\begin{array}{l}\text { Moderate sample } \\
\text { size }(30-49)\end{array}$ & $\begin{array}{l}\text { Small sample } \\
\text { size }(<30)\end{array}$ \\
\hline 4 & Were at least two measurements available? & $\begin{array}{l}\text { At least two } \\
\text { measurements }\end{array}$ & & & $\begin{array}{l}\text { Only one } \\
\text { measurement }\end{array}$ \\
\hline 5 & Were the administrations independent? & $\begin{array}{l}\text { Independent } \\
\text { measurements }\end{array}$ & $\begin{array}{l}\text { Assumable that } \\
\text { the measurements } \\
\text { were independent }\end{array}$ & $\begin{array}{l}\text { Doubtful whether } \\
\text { the measurements } \\
\text { were independent }\end{array}$ & $\begin{array}{l}\text { measurements } \\
\text { NOT } \\
\text { independent }\end{array}$ \\
\hline 6 & Was the time interval stated? & $\begin{array}{l}\text { Time interval } \\
\text { stated }\end{array}$ & & $\begin{array}{l}\text { Time interval NOT } \\
\text { stated }\end{array}$ & \\
\hline 7 & Were patients stable in the interim period on the construct to be measured? & $\begin{array}{l}\text { Patients were } \\
\text { stable (evidence } \\
\text { provided) }\end{array}$ & $\begin{array}{l}\text { Assumable that } \\
\text { patients were } \\
\text { stable }\end{array}$ & $\begin{array}{l}\text { Unclear if patients } \\
\text { were stable }\end{array}$ & $\begin{array}{l}\text { Patients were } \\
\text { NOT stable }\end{array}$ \\
\hline 8 & Was the time interval appropriate? & $\begin{array}{l}\text { Time interval } \\
\text { appropriate }\end{array}$ & & $\begin{array}{l}\text { Doubtful whether } \\
\text { time interval was } \\
\text { appropriate }\end{array}$ & $\begin{array}{l}\text { Time interval } \\
\text { NOT } \\
\text { appropriate }\end{array}$ \\
\hline
\end{tabular}




\begin{tabular}{|c|c|c|c|c|c|}
\hline 9 & $\begin{array}{l}\text { Were the test conditions similar for both measurements? e.g. type of administration, } \\
\text { environment, instructions }\end{array}$ & $\begin{array}{l}\text { Test conditions } \\
\text { were similar } \\
\text { (evidence } \\
\text { provided) }\end{array}$ & $\begin{array}{l}\text { Assumable that } \\
\text { test conditions } \\
\text { were similar }\end{array}$ & $\begin{array}{l}\text { Unclear if test } \\
\text { conditions were } \\
\text { similar }\end{array}$ & $\begin{array}{l}\text { Test conditions } \\
\text { were NOT } \\
\text { similar }\end{array}$ \\
\hline 10 & Were there any important flaws in the design or methods of the study? & $\begin{array}{l}\text { No other important } \\
\text { methodological } \\
\text { flaws in the design } \\
\text { or execution of the } \\
\text { study }\end{array}$ & & $\begin{array}{l}\text { Other minor } \\
\text { methodological } \\
\text { flaws in the design } \\
\text { or execution of the } \\
\text { study }\end{array}$ & $\begin{array}{l}\text { Other important } \\
\text { methodological } \\
\text { flaws in the } \\
\text { design or } \\
\text { execution of the } \\
\text { study }\end{array}$ \\
\hline \multicolumn{6}{|c|}{ Statistical methods } \\
\hline 11 & for continuous scores: Was an intraclass correlation coefficient (ICC) calculated? & $\begin{array}{l}\text { ICC calculated } \\
\text { and model or } \\
\text { formula of the ICC } \\
\text { is described }\end{array}$ & $\begin{array}{l}\text { ICC calculated but } \\
\text { model or formula } \\
\text { of the ICC not } \\
\text { described or not } \\
\text { optimal. } \\
\text { Pearson or } \\
\text { Spearman } \\
\text { correlation } \\
\text { coefficient } \\
\text { calculated with } \\
\text { evidence provided } \\
\text { that no systematic } \\
\text { change has } \\
\text { occurred }\end{array}$ & $\begin{array}{l}\text { Pearson or } \\
\text { Spearman } \\
\text { correlation } \\
\text { coefficient } \\
\text { calculated } \\
\text { WITHOUT } \\
\text { evidence provided } \\
\text { that no systematic } \\
\text { change has } \\
\text { occurred or WITH } \\
\text { evidence that } \\
\text { systematic change } \\
\text { has occurred }\end{array}$ & $\begin{array}{l}\text { No ICC or } \\
\text { Pearson or } \\
\text { Spearman } \\
\text { correlations } \\
\text { calculated }\end{array}$ \\
\hline 12 & for dichotomous/nominal/ordinal scores: Was kappa calculated? & Kappa calculated & & & $\begin{array}{l}\text { Only percentage } \\
\text { agreement } \\
\text { calculated }\end{array}$ \\
\hline 13 & for ordinal scores: Was a weighted kappa calculated? & $\begin{array}{l}\text { Weighted Kappa } \\
\text { calculated }\end{array}$ & & $\begin{array}{l}\text { Unweighted } \\
\text { Kappa calculated }\end{array}$ & $\begin{array}{l}\text { Only percentage } \\
\text { agreement } \\
\text { calculated }\end{array}$ \\
\hline 14 & for ordinal scores: Was the weighting scheme described? e.g. linear, quadratic & $\begin{array}{l}\text { Weighting scheme } \\
\text { described }\end{array}$ & $\begin{array}{l}\text { Weighting scheme } \\
\text { NOT described }\end{array}$ & & \\
\hline \multicolumn{6}{|c|}{ Box C. Measurement error: absolute measures } \\
\hline & & excellent & good & fair & poor \\
\hline \multicolumn{2}{|c|}{ Design requirements } & & & & \\
\hline 1 & Was the percentage of missing items given? & $\begin{array}{l}\text { Percentage of } \\
\text { missing items } \\
\text { described }\end{array}$ & $\begin{array}{l}\text { Percentage of } \\
\text { missing items } \\
\text { NOT described }\end{array}$ & & \\
\hline 2 & Was there a description of how missing items were handled? & $\begin{array}{l}\text { Described how } \\
\text { missing items } \\
\text { were handled }\end{array}$ & $\begin{array}{l}\text { Not described but } \\
\text { it can be deduced } \\
\text { how missing items } \\
\text { were handled }\end{array}$ & $\begin{array}{l}\text { Not clear how } \\
\text { missing items } \\
\text { were handled }\end{array}$ & \\
\hline 3 & Was the sample size included in the analysis adequate? & $\begin{array}{l}\text { Adequate sample } \\
\text { size }(\geq 100)\end{array}$ & $\begin{array}{l}\text { Good sample size } \\
(50-99)\end{array}$ & $\begin{array}{l}\text { Moderate sample } \\
\text { size ( } 30-49)\end{array}$ & $\begin{array}{l}\text { Small sample } \\
\text { size }(<30)\end{array}$ \\
\hline 4 & Were at least two measurements available? & $\begin{array}{l}\text { At least two } \\
\text { measurements }\end{array}$ & & & $\begin{array}{l}\text { Only one } \\
\text { measurement }\end{array}$ \\
\hline 5 & Were the administrations independent? & $\begin{array}{l}\text { Independent } \\
\text { measurements }\end{array}$ & $\begin{array}{l}\text { Assumable that } \\
\text { the measurements } \\
\text { were independent }\end{array}$ & $\begin{array}{l}\text { Doubtful whether } \\
\text { the measurements } \\
\text { were independent }\end{array}$ & $\begin{array}{l}\text { measurements } \\
\text { NOT } \\
\text { independent }\end{array}$ \\
\hline 6 & Was the time interval stated? & $\begin{array}{l}\text { Time interval } \\
\text { stated }\end{array}$ & & $\begin{array}{l}\text { Time interval NOT } \\
\text { stated }\end{array}$ & \\
\hline 7 & Were patients stable in the interim period on the construct to be measured? & $\begin{array}{l}\text { Patients were } \\
\text { stable (evidence } \\
\text { provided) }\end{array}$ & $\begin{array}{l}\text { Assumable that } \\
\text { patients were } \\
\text { stable }\end{array}$ & $\begin{array}{l}\text { Unclear if patients } \\
\text { were stable }\end{array}$ & $\begin{array}{l}\text { Patients were } \\
\text { NOT stable }\end{array}$ \\
\hline 8 & Was the time interval appropriate? & $\begin{array}{l}\text { Time interval } \\
\text { appropriate }\end{array}$ & & $\begin{array}{l}\text { Doubtful whether } \\
\text { time interval was } \\
\text { appropriate }\end{array}$ & $\begin{array}{l}\text { Time interval } \\
\text { NOT } \\
\text { appropriate }\end{array}$ \\
\hline 9 & $\begin{array}{l}\text { Were the test conditions similar for both measurements? e.g. type of administration, } \\
\text { environment, instructions }\end{array}$ & $\begin{array}{l}\text { Test conditions } \\
\text { were similar } \\
\text { (evidence } \\
\text { provided) }\end{array}$ & $\begin{array}{l}\text { Assumable that } \\
\text { test conditions } \\
\text { were similar }\end{array}$ & $\begin{array}{l}\text { Unclear if test } \\
\text { conditions were } \\
\text { similar }\end{array}$ & $\begin{array}{l}\text { Test conditions } \\
\text { were NOT } \\
\text { similar }\end{array}$ \\
\hline 10 & Were there any important flaws in the design or methods of the study? & $\begin{array}{l}\text { No other important } \\
\text { methodological } \\
\text { flaws in the design } \\
\text { or execution of the } \\
\text { study }\end{array}$ & & $\begin{array}{l}\text { Other minor } \\
\text { methodological } \\
\text { flaws in the design } \\
\text { or execution of the } \\
\text { study }\end{array}$ & $\begin{array}{l}\text { Other important } \\
\text { methodological } \\
\text { flaws in the } \\
\text { design or } \\
\text { execution of the } \\
\text { study }\end{array}$ \\
\hline \multicolumn{6}{|c|}{ Statistical methods } \\
\hline 11 & $\begin{array}{l}\text { for CTT: Was the Standard Error of Measurement (SEM), Smallest Detectable } \\
\text { Change (SDC) or Limits of Agreement (LoA) calculated? }\end{array}$ & $\begin{array}{l}\text { SEM, SDC, or } \\
\text { LoA calculated }\end{array}$ & $\begin{array}{l}\text { Possible to } \\
\text { calculate LoA from } \\
\text { the data } \\
\text { presented }\end{array}$ & & $\begin{array}{l}\text { SEM calculated } \\
\text { based on } \\
\text { Cronbach's } \\
\text { alpha, or on SD } \\
\text { from another } \\
\text { population }\end{array}$ \\
\hline \multicolumn{6}{|c|}{ Box D. Content validity (including face validity) } \\
\hline & & excellent & good & fair & poor \\
\hline \multicolumn{2}{|c|}{ General requirements } & & & & \\
\hline 1 & $\begin{array}{l}\text { Was there an assessment of whether all items refer to relevant aspects of the } \\
\text { construct to be measured? }\end{array}$ & $\begin{array}{l}\text { Assessed if all } \\
\text { items refer to } \\
\text { relevant aspects } \\
\text { of the construct to } \\
\text { be measured }\end{array}$ & & $\begin{array}{l}\text { Aspects of the } \\
\text { construct to be } \\
\text { measured poorly } \\
\text { described AND } \\
\text { this was not taken } \\
\text { into consideration }\end{array}$ & $\begin{array}{l}\text { NOT assessed if } \\
\text { all items refer to } \\
\text { relevant aspects } \\
\text { of the construct } \\
\text { to be measured }\end{array}$ \\
\hline
\end{tabular}




\begin{tabular}{|c|c|c|c|c|c|}
\hline \multirow[b]{2}{*}{2} & \multirow[b]{2}{*}{$\begin{array}{l}\text { Was there an assessment of whether all items are relevant for the study } \\
\text { population? (e.g. age, gender, disease characteristics, country, setting) }\end{array}$} & \multirow[b]{2}{*}{$\begin{array}{l}\text { Assessed if all } \\
\text { items are relevant } \\
\text { for the study } \\
\text { population in } \\
\text { adequate sample } \\
\text { size }(\geq 10)\end{array}$} & \\
\hline & & & $\begin{array}{l}\text { Assessed if all } \\
\text { items are relevant } \\
\text { for the study } \\
\text { population in } \\
\text { moderate sample } \\
\text { size (5-9) }\end{array}$ & $\begin{array}{l}\text { Assessed if all } \\
\text { items are relevant } \\
\text { for the study } \\
\text { population in small } \\
\text { sample size }(<5)\end{array}$ & $\begin{array}{l}\text { NOT assessed if } \\
\text { all items are } \\
\text { relevant for the } \\
\text { study population } \\
\text { OR target } \\
\text { population not } \\
\text { involved }\end{array}$ \\
\hline 3 & $\begin{array}{l}\text { Was there an assessment of whether all items are relevant for the purpose of the } \\
\text { measurement instrument? (discriminative, evaluative, and/or predictive) }\end{array}$ & $\begin{array}{l}\text { Assessed if all } \\
\text { items are relevant } \\
\text { for the purpose of } \\
\text { the application }\end{array}$ & $\begin{array}{l}\text { Purpose of the } \\
\text { instrument was } \\
\text { not described but } \\
\text { assumed }\end{array}$ & $\begin{array}{l}\text { NOT assessed if } \\
\text { all items are } \\
\text { relevant for the } \\
\text { purpose of the } \\
\text { application }\end{array}$ & \\
\hline 4 & $\begin{array}{l}\text { Was there an assessment of whether all items together comprehensively reflect the } \\
\text { construct to be measured? }\end{array}$ & $\begin{array}{l}\text { Assessed if all } \\
\text { items together } \\
\text { comprehensively } \\
\text { reflect the } \\
\text { construct to be } \\
\text { measured }\end{array}$ & & $\begin{array}{l}\text { No theoretical } \\
\text { foundation of the } \\
\text { construct and this } \\
\text { was not taken into } \\
\text { consideration }\end{array}$ & $\begin{array}{l}\text { NOT assessed if } \\
\text { all items } \\
\text { together } \\
\text { comprehen- } \\
\text { sively reflect the } \\
\text { construct to be } \\
\text { measured }\end{array}$ \\
\hline 5 & Were there any important flaws in the design or methods of the study? & $\begin{array}{l}\text { No other important } \\
\text { methodological } \\
\text { flaws in the design } \\
\text { or execution of the } \\
\text { study }\end{array}$ & & $\begin{array}{l}\text { Other minor } \\
\text { methodological } \\
\text { flaws in the design } \\
\text { or execution of the } \\
\text { study }\end{array}$ & $\begin{array}{l}\text { Other important } \\
\text { methodological } \\
\text { flaws in the } \\
\text { design or } \\
\text { execution of the } \\
\text { study }\end{array}$ \\
\hline \multicolumn{6}{|c|}{ Box E. Structural validity } \\
\hline & & excellent & good & fair & poor \\
\hline \multicolumn{6}{|c|}{ Design requirements } \\
\hline 2 & Was the percentage of missing items given? & $\begin{array}{l}\text { Percentage of } \\
\text { missing items } \\
\text { described }\end{array}$ & $\begin{array}{l}\text { Percentage of } \\
\text { missing items } \\
\text { NOT described }\end{array}$ & & \\
\hline 3 & Was there a description of how missing items were handled? & $\begin{array}{l}\text { Described how } \\
\text { missing items } \\
\text { were handled }\end{array}$ & $\begin{array}{l}\text { Not described but } \\
\text { it can be deduced } \\
\text { how missing items } \\
\text { were handled }\end{array}$ & $\begin{array}{l}\text { Not clear how } \\
\text { missing items } \\
\text { were handled }\end{array}$ & \\
\hline 4 & Was the sample size included in the analysis adequate? & $\begin{array}{l}7^{*} \text { \#items and } \\
\geq 100\end{array}$ & $\begin{array}{l}5^{\star} \# \text { items and } \\
\geq 100 \text { OR } 5-7^{*} \\
\# \text { items but }<100\end{array}$ & $\begin{array}{l}5^{\star} \# \text { \#items but } \\
<100\end{array}$ & $<5^{\star}$ \#items \\
\hline 5 & Were there any important flaws in the design or methods of the study? & $\begin{array}{l}\text { No other important } \\
\text { methodological } \\
\text { flaws in the design } \\
\text { or execution of the } \\
\text { study }\end{array}$ & & $\begin{array}{l}\text { Other minor } \\
\text { methodological } \\
\text { flaws in the design } \\
\text { or execution of the } \\
\text { study (e.g. rotation } \\
\text { method not } \\
\text { described) }\end{array}$ & $\begin{array}{l}\text { Other important } \\
\text { methodological } \\
\text { flaws in the } \\
\text { design or } \\
\text { execution of the } \\
\text { study (e.g. } \\
\text { inappropriate } \\
\text { rotation method) }\end{array}$ \\
\hline \multicolumn{6}{|c|}{ Statistical methods } \\
\hline 6 & for CTT: Was exploratory or confirmatory factor analysis performed? & $\begin{array}{l}\text { Exploratory or } \\
\text { confirmatory factor } \\
\text { analysis } \\
\text { performed and } \\
\text { type of factor } \\
\text { analysis } \\
\text { appropriate in } \\
\text { view of existing } \\
\text { information }\end{array}$ & $\begin{array}{l}\text { Exploratory factor } \\
\text { analysis } \\
\text { performed while } \\
\text { confirmatory } \\
\text { would have been } \\
\text { more appropriate }\end{array}$ & & $\begin{array}{l}\text { No exploratory } \\
\text { or confirmatory } \\
\text { factor analysis } \\
\text { performed }\end{array}$ \\
\hline 7 & $\begin{array}{l}\text { for IRT: Were IRT tests for determining the (uni-) dimensionality of the items } \\
\text { performed? }\end{array}$ & $\begin{array}{l}\text { IRT test for } \\
\text { determining } \\
\text { (uni)dimension- } \\
\text { ality performed }\end{array}$ & & & $\begin{array}{l}\text { IRT test for } \\
\text { determining } \\
\text { (uni)dimension- } \\
\text { ality NOT } \\
\text { performed }\end{array}$ \\
\hline
\end{tabular}

\section{Interpretability}

We recommend to use the Interpretability box to extract all information on the interpretability issues described in this box of the instruments under study from the included articles.

\begin{tabular}{|l|l|}
\hline Box Interpretability \\
\hline Percentage of missing items & \multicolumn{2}{|l|}{} \\
\hline Description of how missing items were handled & \\
\hline Distribution of the (total) scores & \\
\hline Percentage of the respondents who had the lowest possible (total) score & \\
\hline Percentage of the respondents who had the highest possible (total) score & \\
\hline $\begin{array}{l}\text { Scores and change scores (i.e. means and SD) for relevant (sub) groups, e.g. for normative } \\
\text { groups, subgroups of patients, or the general population }\end{array}$ & \\
\hline Minimal Important Change (MIC) or Minimal Important Difference (MID) & \\
\hline
\end{tabular}




\section{Box J. Interpretability}

yes no ?

1 Was the percentage of missing items given?

2 Was there a description of how missing items were handled?

3 Was the sample size included in the analysis adequate?

4 Was the distribution of the (total) scores in the study sample described?

5 Was the percentage of the respondents who had the lowest possible (total) score described?

6 Was the percentage of the respondents who had the highest possible (total) score described?

7 Were scores and change scores (i.e. means and SD) presented for relevant (sub) groups? e.g. for normative groups, subgroups of patients, or the general population

8 Was the minimal important change (MIC) or the minimal important difference (MID) determined?

9 Were there any important flaws in the design or methods of the study? 


\section{$7 \quad$ Literaturverzeichnis}

Benrath J, Hatzenbühler M, Fresenius M, Heck M: Repetitorium Schmerztherapie: Zur Vorbereitung auf die Prüfung Spezielle Schmerztherapie. 3., vollständig überarbeitete Auflage; Springer-Verlag, Berlin 2012

Bernateck M, Karst M, Sabatowski R, Siebrecht D (Hrsg.): Schmerzmedizin - 1000 Fragen: Für Klinik, Praxis und die Zusatzweiterbildung Spezielle Schmerztherapie. 2. Auflage; Georg Thieme Verlag, Stuttgart 2017

Böhmer AB, Poels M, Simanski C, Trojan S, Messer K, Wirtz MD, Neugebauer EAM, Wappler F, Joppich R (2012): Qualitätsmanagement in der Akutschmerztherapie: Ergebnisse einer Befragung TÜV-zertifizierter Kliniken. Schmerz $\underline{26}, 425-434$

Bornemann R, Gaber E: Gastritis, Magen- und Zwölffingerdarmgeschwüre (Gesundheitsberichterstattung des Bundes). Band 55; Robert Koch-Institut, Berlin 2013

DGAI (2007): Behandlung akuter perioperativer und posttraumatischer Schmerzen (Gültigkeit abgelaufen - aktuell in Überarbeitung). S3-Leitlinie der Deutsche Gesellschaft für Anästhesiologie und Intensivmedizin. http://www.awmf.org/uploads/tx_szleitlinien/001-025I_S3_Behandlung_akuter_perioperativer_und_posttraumatischer_Schmerzen_abgelaufen.pdf; abgerufen am 15.8.2017

DGP (2017): Epidemiologie, Diagnostik und Therapie erwachsener Patienten mit nosokomialer Pneumonie. S3-Leitlinie der Deutsche Gesellschaft für Pneumologie und Beatmungsmedizin. http://www.awmf.org/leitlinien/detail/II/020-013.html; abgerufen am 26.11.2017

Diener H-C, Maier C, Rolke R: Schmerzbegriffe. In: Diener H-C, Maier C (Hrsg.): Die Schmerztherapie: interdisziplinäre Diagnose- und Behandlungsstrategien. 4. Auflage; Elsevier, Urban \& Fischer, München 2011, 3-6

Erlenwein J (2016): Qualität der Schmerztherapie in Deutschland - Organisation der Akutschmerztherapie: Leitlinien, Empfehlungen und aktuelle Praxis. Anästhesiol Intensivmed Notfallmed Schmerzther 51, 40-48

Ettorchi-Tardy A, Levif M, Michel P (2012): Benchmarking: a method for continuous quality improvement in health. Healthc Policy $\underline{7}$, e101-119

Gerbershagen HJ, Rothaug J, Kalkman CJ, Meissner W (2011): Determination of moderate-to-severe postoperative pain on the numeric rating scale: a cut-off point analysis applying four different methods. Br J Anaesth $107,619-626$

Gerbershagen HJ, Pogatzki-Zahn E, Aduckathil S, Peelen LM, Kappen TH, van Wijck AJM, Kalkman CJ, Meissner W (2014): Procedure-specific risk factor analysis for the development of severe postoperative pain. Anesthesiology $\underline{120}, 1237-1245$

Häuser W, Schmutzer G, Henningsen P, Brähler E (2014): Chronische Schmerzen, Schmerzkrankheit und Zufriedenheit der Betroffenen mit der Schmerzbehandlung in Deutschland. Schmerz 28, 483-492

Heck M, Fresenius M, Busch C (Hrsg.): Repetitorium Anästhesiologie: für die Facharztprüfung und das Europäische Diplom. 8., komplett überarbeitete Auflage; Springer, Berlin 2017

Höllt V, Allgaier C: Analgetika. In: Aktories K, Förstermann U, Hofmann F, Starke K (Hrsg.): Allgemeine und spezielle Pharmakologie und Toxikologie: für Studenten der Medizin, Veterinärmedizin, Pharmazie, Chemie und Biologie sowie für Ärzte, Tierärzte und Apotheker. 11., überarb. Auflage; Elsevier, Urban \& Fischer, München 2013, 207-232

Kindler D, Maier C: Postoperative Schmerztherapie bei Patienten mit chronischen Schmerzen und Tumorerkrankungen. In: Pogatzki-Zahn E, Zahn PK, Van Aken HK (Hrsg.): Postoperative Schmerztherapie (Pathophysiologie, Pharmakologie und Therapie). 1. Auflage; Thieme Verlag, Stuttgart 2008, 238-258

Korczak D, Kuczera C, Rust M (2013): Akutschmerztherapie auf operativen und konservativen Stationen. GMS Health Technol Assess $\underline{126}$

Kutzer K (2008): Recht auf Schmerzfreiheit? - Juristische Aspekte. Dtsch Med Wochenschr 133, 317-320

Larsen R: Anästhesie. 10. Auflage; Elsevier, Urban \& Fischer, München 2013 
Larsen R, Fink T, Müller-Wolf T: Anästhesie und Intensivmedizin für die Fachpflege. 9., vollst. überarb. Auflage; Springer, Berlin 2016

Maier C, Nestler N, Richter H, Hardinghaus W, Pogatzki-Zahn E, Zenz M, Osterbrink J (2010): Qualität der Schmerztherapie in deutschen Krankenhäusern. Dtsch Arztebl Int 107, 607-614

Maier C, Nestler N, Hansel N, Hardinghaus W, Nauck F, Osterbrink J, Pogatzki-Zahn E (2013): Zertifizierung für „Qualifizierte Schmerztherapie“ - Nachhaltige Verbesserung der Versorgungsrealität in deutschen Krankenhäusern. Klinikarzt $\underline{42}, 80-87$

McCaffery M, Beebe A, Latham J: Schmerz. Ein Handbuch für die Pflegepraxis. Ullstein Mosby, Wiesbaden 1997

Meißner W (Hrsg.): Akutschmerz Taschenbuch - Konzepte, Methoden, Praxis. 2., aktualisierte und erweiterte Auflage; MWV Medizinisch Wissenschaftliche Verlagsgesellschaft, Berlin 2015

Meißner W (2016): Qualität der Schmerztherapie in Deutschland - Qualitätsmanagement und -sicherung in der Akutschmerztherapie. Anästhesiol Intensivmed Notfallmed Schmerzther 51, 50-55

Meissner W, Ullrich K, Zwacka S (2006): Benchmarking as a tool of continuous quality improvement in postoperative pain management. Eur J Anaesthesiol 23, 142-148

Meissner W, Mescha S, Rothaug J, Zwacka S, Goettermann A, Ulrich K, Schleppers A (2008): Qualitätsverbesserung der postoperativen Schmerztherapie Ergebnisse des QUIPS-Projekts. Dtsch Arztebl Int 105, 865-870

Menche N, Asmussen-Clausen M: Pflege heute: Lehrbuch für Pflegeberufe. 5., vollst. überarb. Auflage; Elsevier, Urban \& Fischer, München 2011

Mokkink LB, Terwee CB, Knol DL, Stratford PW, Alonso J, Patrick DL, Bouter LM, de Vet HCW (2006): Protocol of the COSMIN study: COnsensus-based Standards for the selection of health Measurement INstruments. BMC Med Res Methodol $\underline{6}, 2$

Mokkink LB, Terwee CB, Patrick DL, Alonso J, Stratford PW, Knol DL, Bouter LM, de Vet HCW (2010a): The COSMIN checklist for assessing the methodological quality of studies on measurement properties of health status measurement instruments: an international Delphi study. Qual Life Res $\underline{19}$, 539-549

Mokkink LB, Terwee CB, Knol DL, Stratford PW, Alonso J, Patrick DL, Bouter LM, de Vet HC (2010b): The COSMIN checklist for evaluating the methodological quality of studies on measurement properties: a clarification of its content. BMC Med Res Methodol $\underline{10}, 22$

(Muster-)Berufsordnung (2015): „Berufsordnung“ der Bundesärztekammer für die in Deutschland tätigen Ärztinnen und Ärzte in der Fassung des Beschlusses des 118. Deutschen Ärztetages 2015 in Frankfurt am Main, zuletzt geändert am 14.12.2018.

Nau C (2010): Chronische Schmerzen nach Operationen - Pathophysiologie und prädisponierende Faktoren. Anästhesiol Intensivmed Notfallmed Schmerzther $\underline{45}$, 480-487

Nestler N, Osterbrink J (2009): PS4. Haben Schulungen von Pflegenden Einfluss auf das Schmerzmanagement? Periop Med 1, 254-255

Nestler N, Gnass I, Pogatzki-Zahn E, Osterbrink J (2013): Der Weg zu einem interprofessionellen Schmerzmanagement im Krankenhaus - Beispiel des Aktionsbündnisses Schmerzfreie Stadt Münster. Klinikarzt 42, 88-94

Nobis H-G, Rolke R, Graf-Baumann T (Hrsg.): Schmerz - eine Herausforderung: Informationen für Betroffene und Angehörige - Offizielle Informationsschrift mehrerer Schmerzgesellschaften. 2. Auflage; Springer-Verlag, Berlin 2016

Pogatzki-Zahn E (2010): Chronische Schmerzen nach Operationen - Prävention und Therapie. Anästhesiol Intensivmed Notfallmed Schmerzther $\underline{45}, 496-504$

Pogatzki-Zahn E (2011): Therapie und Prävention postoperativer Schmerzen bei chronischen Schmerzpatienten. Anästh Intensivmed 52, 388-404 
Pogatzki-Zahn EM, Van Aken HK, Zahn PK (Hrsg.): Postoperative Schmerztherapie: Pathophysiologie, Pharmakologie und Therapie. 1. Auflage; Georg Thieme Verlag, Stuttgart 2008

Rothaug J: Schmerzerfassung in der postoperativen Schmerztherapie: psychometrische Qualität von QUIPS und PAIN OUT. Med. Diss. Jena 2013

Rothaug J, Weiss T, Meissner W (2012): Externe Validität der schmerzbedingten Funktionsbeeinträchtigung. Schmerz 26, 396-401

Rothaug J, Zaslansky R, Schwenkglenks M, Komann M, Allvin R, Backström R, Brill S, Buchholz I, Engel C, Fletcher D, et al. (2013): Patients' perception of postoperative pain management: validation of the International Pain Outcomes (IPO) questionnaire. J Pain 14, 1361-1370

Schmidt RF, Lang F, Heckmann M: Physiologie des Menschen: mit Pathophysiologie. 31., überarb. und aktualisierte Auflage; Springer Medizin-Verlag, Heidelberg 2010

Schnabel A, Pogatzki-Zahn E (2010): Prädiktoren für chronische Schmerzen nach Operationen. Schmerz 24, 517-533

Speckmann E-J, Hescheler J, Köhling R, Alzheimer C: Physiologie. 6. Auflage; Elsevier, Urban \& Fischer, München 2013

Striebel HW: Anästhesie, Intensivmedizin, Notfallmedizin: für Studium und Ausbildung. 9., vollständig überarbeitete Auflage; Schattauer, Stuttgart 2017

Terwee CB, Mokkink LB, Knol DL, Ostelo RWJG, Bouter LM, de Vet HCW (2012): Rating the methodological quality in systematic reviews of studies on measurement properties: a scoring system for the COSMIN checklist. Qual Life Res $\underline{21}, 651-657$

Trepel M: Neuroanatomie: Struktur und Funktion. 5. Auflage; Elsevier, Urban \& Fischer, München 2012

Woolf CJ (2011): Central sensitization: Implications for the diagnosis and treatment of pain. Pain $\underline{152}$, S2-15

Zaslansky R, Rothaug J, Chapman RC, Backström R, Brill S, Engel C, Fletcher D, Fodor L, Funk P, Gordon D, et al. (2014): PAIN OUT: an international acute pain registry supporting clinicians in decision making and in quality improvement activities. J Eval Clin Pract 20, 1090-1098

Zaslansky R, Rothaug J, Chapman CR, Bäckström R, Brill S, Fletcher D, Fodor L, Gordon DB, Komann M, Konrad C, et al. (2015): PAIN OUT: the making of an international acute pain registry. Eur J Pain $\underline{19}, 490-502$

\section{Internetquellen}

Gabler Wirtschaftslexikon - Benchmarking. http://wirtschaftslexikon.gabler.de/Definition/benchmarking.html; Zugriff am 06.09.2017

IASP Terminology. https://www.iasp-pain.org/Education/Content.aspx?ItemNumber=1698; Zugriff am 25.03.2020

QUIPS-Homepage. http://quips-projekt.de/de/was-ist-quips; Zugriff am 01.01.2017 


\section{Danksagung}

Mein besonderer Dank gilt meinem Doktorvater Prof. Dr. med. Frank Petzke, da er mir diese Promotionsarbeit ermöglicht hat und mich bei allen Fragen oder Problemen immer konstruktiv und wegweisend geleitet hat.

Zudem möchte ich mich auch bei meinem Zweitbetreuer PD Dr. med. Steffen Kunsch bedanken, der das Projekt jederzeit konstruktiv unterstützt und beraten hat.

Für seine persönliche Betreuung meiner Arbeit möchte ich auch PD Dr. med. Joachim Erlenwein danken, der meine Promotionsarbeit ebenfalls maßgeblich begleitet hat.

Ein großer Dank gilt auch Prof. Dr. med. Winfried Meißner, dem Leiter des QUIPS-Projekts, sowie Antje Göttermann und Dr. Ing. Marcus Komann aus Jena und Dr. med. Lars Sturm und Sylvia Schulz aus dem Krankenhaus Martha-Maria in Halle-Dölau.

Für ihre fachspezifische Beratung danke ich Prof. Dr. med. Martin Sommer, Klinik für Klinische Neurophysiologie und Dr. med. Kai-Martin Thoms, Klinik für Dermatologie, Venerologie und Allergologie jeweils an der Universitätsmedizin Göttingen. Außerdem bedanke ich mich bei allen Direktoren und Chefärzten der Universitätsklinik Jena, dem Krankenhaus Martha-Maria Halle-Dölau und der Universitätsmedizin Göttingen, auf deren Stationen freundlicherweise Patienten eingeschlossen werden durften und die damit das Projekt maßgeblich unterstützt haben.

Auch hätte das Projekt nicht ohne die tatkräftige Unterstützung der MitarbeiterInnen in der UMG Schmerzmedizin, sowie in den Abteilungen für die Bettenbelegung der einzelnen Kliniken abgeschlossen werden können. 